\title{
Beitrag der Ganzkörper-MRT \\ in der Beurteilung von Osteonekrosen bei Kindern und Jugendlichen mit onkologischen und hämatologischen Grunderkrankungen
}

\author{
Dissertation \\ zur Erlangung des akademischen Grades \\ doctor medicinae (Dr. med.)
}

vorgelegt dem Rat der Medizinischen Fakultät der Friedrich-Schiller-Universität Jena

von Miriam Korch

geboren am 25. Januar 1993 in Räckelwitz 


\section{Gutachter}

1. Prof. Dr. med. Hans-Joachim Mentzel, Jena

2. Prof. Dr. med. James Beck, Jena

3. Prof. Dr. med. Meinrad Beer, UIm

Tag der öffentlichen Verteidigung: 09.04.2018 


\section{Abkürzungsverzeichnis}

2D

zweidimensional

$3 \mathrm{D}$

dreidimensional

$\alpha$

Signifikanzniveau

ADC

Apparent Diffusion Coefficient

ALL

Akute Lymphatische Leukämie

AML

Akute Myeloische Leukämie

ARCO Association Research Circulation Osseous

bzw. beziehungsweise

$\mathrm{cm} \quad$ Zentimeter

CML Chronisch Myeloische Leukämie

CT Computertomographie

CUP Cancer of unknown primary

d.h. das heißt

DTPA Diethylentriaminpentaessigsäure

DWI Diffusion Weighted Imaging = Diffusionswichtende Bildgebung

FLAIR Fluid Attenuated Inversion Recovery

g Gramm

GK-MRT Ganzkörper-Magnetresonanztomographie

GvHD Graft-versus-Host-Disease

HASTE Half Fourier Acquired Single Shot Turbo Spin Echo

HASTE-IR Half Fourier Acquired Single Shot Turbo Spin Echo mit Inversion

Recocery

HR Hazard Ratio

i.v. intravenös

$\mathrm{kg} \quad$ Kilogramm

KG Körpergewicht

m Meter

mg Milligramm

$\mathrm{ml} \quad$ Milliliter

$\mathrm{mm} \quad$ Millimeter

mmol Millimol

MR Magnetresonanz

MRT Magnetresonanztomographie 


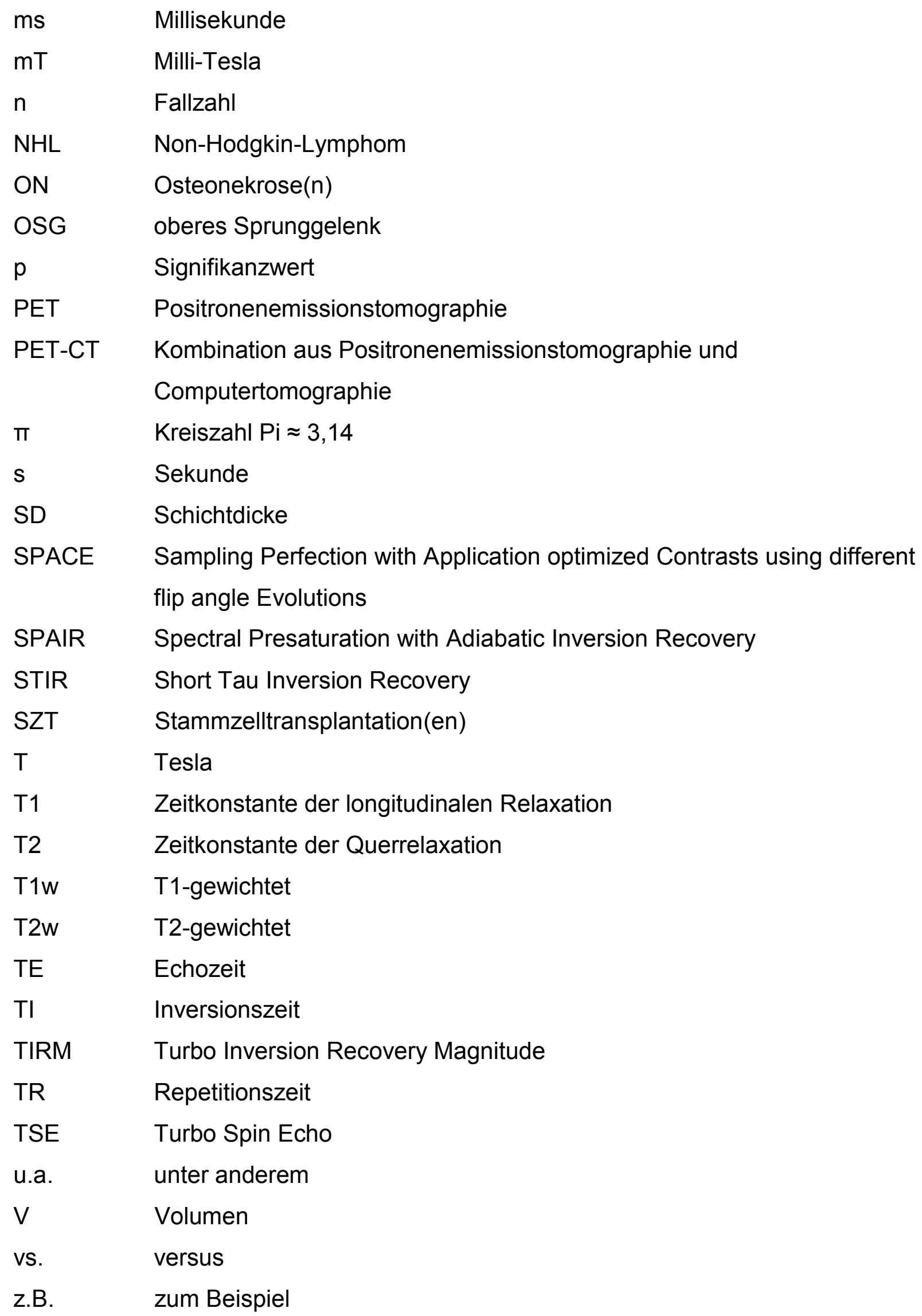




\section{Inhaltsverzeichnis}

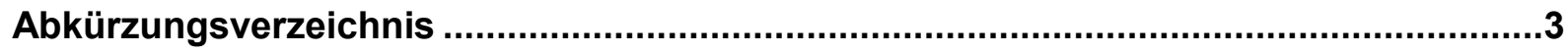

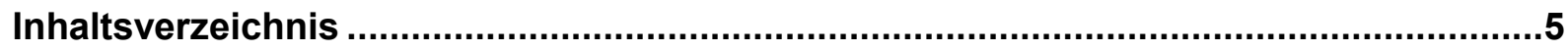

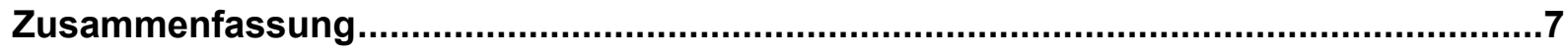

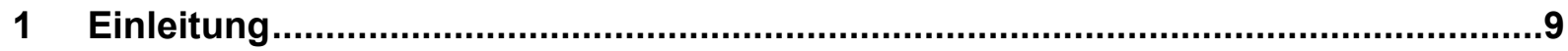

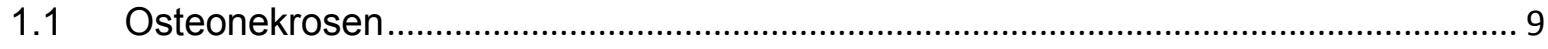

1.1.1 Pathogenese und Ursachen der Osteonekrosenentstehung.............................. 9

1.1.2 Inzidenz, Symptomatik und Lokalisationen der Osteonekrosen ........................ 11

1.1.3 Risikofaktoren für die Entwicklung von Osteonekrosen ..................................... 11

1.1.4 Verlauf und Komplikationen der Osteonekrosen ................................................ 13

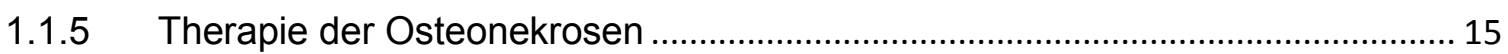

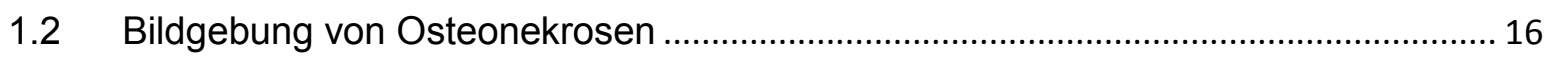

1.2.1 Mögliche MRT-Sequenzen zur Darstellung von Osteonekrosen ......................... 16

1.2.2 Nutzen der MRT zur Detektion von Osteonekrosen ............................................. 17

1.2.3 Differentialdiagnosen der Osteonekrosen in der MRT ......................................... 19

1.3 Ganzkörper-Magnetresonanztomographie im Kindesalter ........................................ 19

1.3.1 Entwicklung der GK-MRT und Möglichkeiten in der Pädiatrie ............................. 19

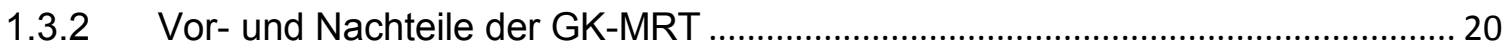

1.3.3 Konzepte und technische Aspekte zur Durchführung einer GK-MRT ................ 22

1.3.4 Indikationen für die GK-MRT-Bildgebung in der Pädiatrie .................................. 23

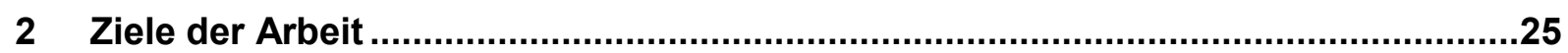

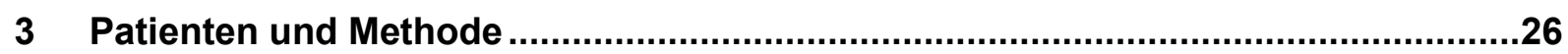

3.1 Auswahl und Einteilung der Patienten...................................................................... 26

3.1.1 Patienten ohne Stammzelltransplantation ............................................................ 27

3.1.2 Patienten mit Stammzelltransplantation.............................................................. 28

3.2 Bildgebung mittels Ganzkörper-Magnetresonanztomographie ................................. 29

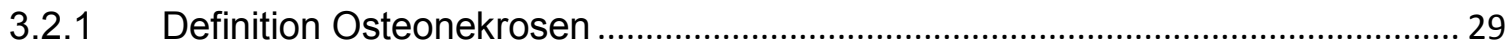

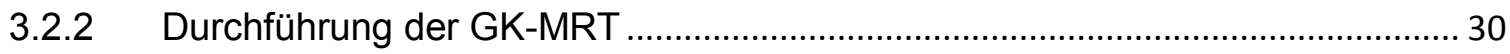

3.2.3 Qualitätsbewertung zur Darstellung von Osteonekrosen .................................... 32

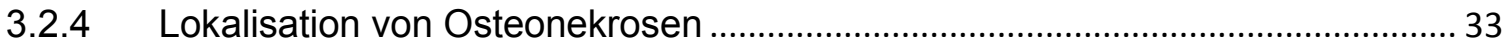

3.2.5 Patientenspezifische Einflussfaktoren auf den positiven Nachweis von

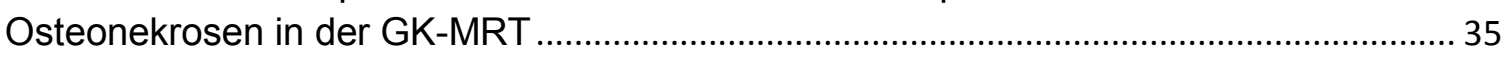

3.2.6 Zeitlicher Verlauf der Osteonekrosen ..................................................................... 36

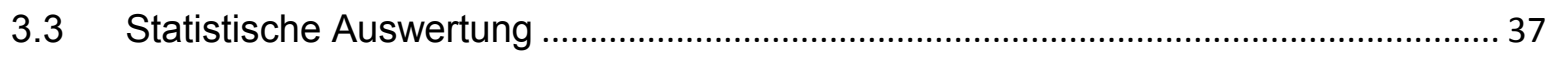


4.1 Qualitätsbewertung der einzelnen Sequenzen im Nachweis von Osteonekrosen ... 38

4.2 Positiver Nachweis von Osteonekrosen in der GK-MRT und Bezug zu klinischer Symptomatik

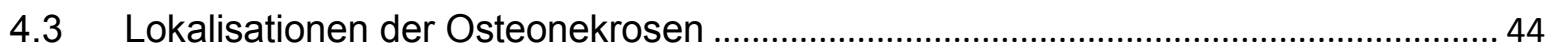

4.3.1 Anzahl und Verteilung der Lokalisationen der Osteonekrosen ............................ 44

4.3.2 Häufigkeit betroffener Knochen und gelenknaher Knochenregionen .................. 45

4.3.3 Osteonekrosen in den das Kniegelenk bildenden Knochen ................................. 47

4.3.4 Lage der Osteonekrosen in den langen Röhrenknochen ..................................... 48

4.4 Einflussfaktoren auf das Auftreten von Osteonekrosen ............................................ 50

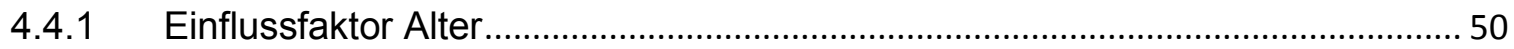

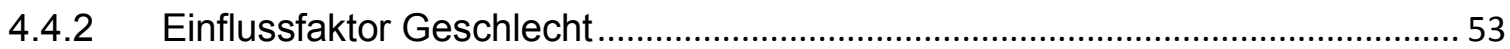

4.4.3 Einflussfaktor Art des verabreichten Steroids und Höhe der kumulativen

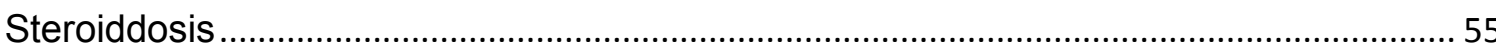

4.5 Zeitlicher Verlauf der Osteonekrosen bei Patienten mit Morbus Hodgkin ..................60 60

4.5.1 Vergleich der Verläufe der Patienten mit Morbus Hodgkin ................................... 61

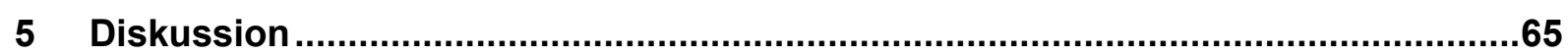

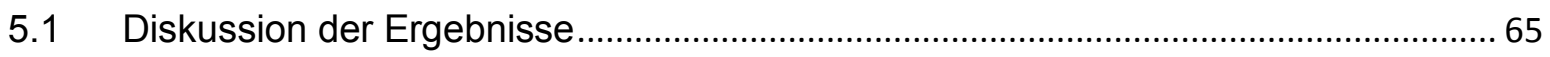

5.1.1 Mögliche Sequenzen zur Darstellung von Osteonekrosen .................................. 65

5.1.2 Häufigkeit der Osteonekrosen und Symptomatik ............................................... 70

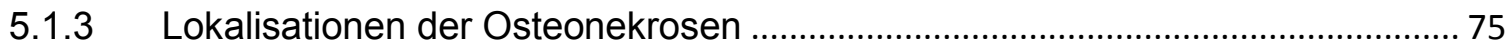

5.1.4 Einflussfaktor Alter auf das Auftreten von Osteonekrosen.................................. 77

5.1.5 Einflussfaktor Geschlecht auf das Auftreten von Osteonekrosen....................... 79

5.1.6 Einflussfaktor Steroide auf das Auftreten von Osteonekrosen ............................. 80

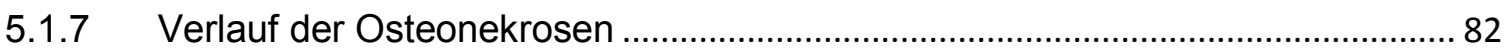

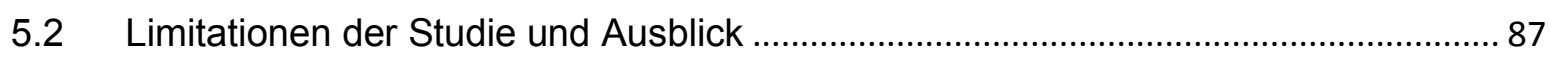

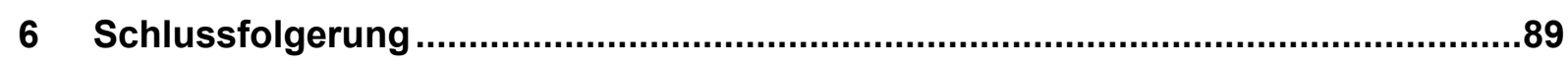

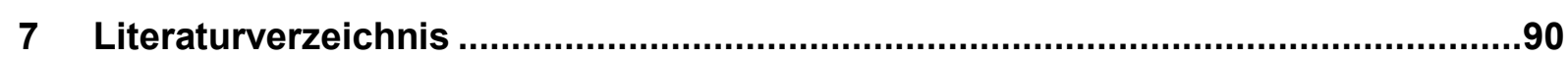

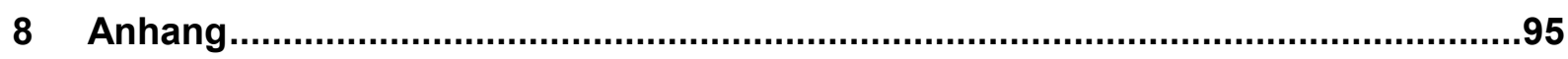

8.1 Zeitlicher Verlauf der Osteonekrosen bei den einzelnen Patienten............................. 95

8.1.1 Patienten ohne Behandlung der Osteonekrosen................................................. 95

8.1.2 Patienten mit Behandlung der Osteonekrosen ...................................................... 98

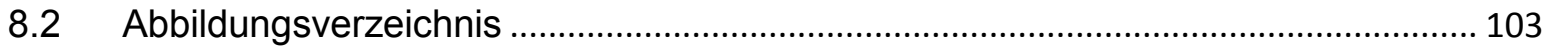

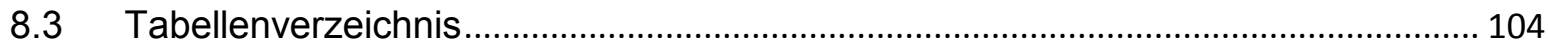

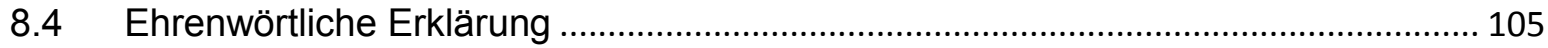




\section{Zusammenfassung}

Ziel: In dieser retrospektiven Studie sollte analysiert werden, wie gut Osteonekrosen (ON) bei Kindern und Jugendlichen in den einzelnen Sequenzen der GanzkörperMagnetresonanztomographie (GK-MRT) dargestellt und quantifiziert werden können, in welcher Lokalisation die Läsionen am häufigsten zu finden sind und welche patientenspezifischen Faktoren das Auftreten von ON in der GK-MRT beeinflussen. Des Weiteren sollte der natürliche Verlauf der ON sowie die Reaktion der Läsionen auf bestimmte Therapien dargestellt werden.

Patienten und Methoden: 105 Kinder und Jugendliche (62 männlich, 43 weiblich) mit onkologischen und hämatologischen Grunderkrankungen, die mindestens eine GK-MRT im Verlauf ihrer Erkrankung erhielten, wurden eingeschlossen. Sie wurden hinsichtlich einer durchgeführten Stammzelltransplantation (SZT) in zwei Gruppen geteilt (63 Patienten ohne SZT: mittleres Alter bei Diagnose der Erkrankung 11,1 \pm 5,6 Jahre, Median = 12,9 Jahre; 42 Patienten mit SZT: mittleres Alter bei der ersten SZT 10,7 \pm 6,5 Jahre, Median = 12,0 Jahre). Die GK-MRT-Sequenzen T1-SPACE, T2-HASTE, T2-HASTE-IR oder T1-SPACE-SPAIR mit Kontrastmittel $(0,1 \mathrm{mmol} / \mathrm{kg}$ KG i.v.) wurden hinsichtlich der Darstellung von ON ausgewertet. Anhand eines Qualitätsscore mit 6 Kriterien sowie dem einzelnen Kriterium „diagnostische Sicherheit" wurden die Sequenzen der jeweils ersten GK-MRT mit Nachweis von ON untereinander in ihrer Aussagekraft verglichen. Alle ON der vorhandenen GK-MRT wurden zur Beurteilung der häufigsten Lokalisationen im Skelett erfasst. Für die Patientengruppen ohne sowie mit SZT wurden der Einfluss des Geschlechts und des Alters bei der Diagnose der Grunderkrankung bzw. bei der ersten SZT auf das Auftreten von ON in der GK-MRT analysiert. Bei den Patienten ohne SZT wurde zusätzlich der Einfluss verabreichter Steroide untersucht. Der zeitliche Verlauf der Summe der ON-Volumina wurde für die Patienten mit der Grunderkrankung Morbus Hodgkin dargestellt.

Ergebnisse: Der Qualitätsscore sowie der Wert der diagnostischen Sicherheit waren in den Sequenzen T1-nativ und T1 mit Kontrastmittel jeweils den Werten der T2Wichtungen signifikant überlegen $(p<0,001)$. Bei 26/63 Patienten ohne SZT $(41,3$ $\%)$ und 17/42 Patienten mit SZT (40,5\%) wurde mindestens eine ON in der GK-MRT 
diagnostiziert. Die mediane Anzahl an ON pro Patient betrug 9,0 (1 - 49 ON/Patient). In beiden Gruppen wurden ON v.a. in der unteren Extremität insbesondere kniegelenknahe verifiziert. Ein Alter von $>10$ Jahren bei der Diagnose der Grunderkrankung bzw. der ersten SZT (Patienten ohne SZT: $p=0,002$; Patienten mit SZT: $p=0,293$ ) sowie weibliches Geschlecht (Patienten ohne SZT: $p=0,477$; Patienten mit SZT: $p=0,379$ ) gingen mit einem höheren Risiko für ein Auftreten von ON in der GK-MRT einher. Patienten ohne SZT, welche Steroide im Rahmen ihrer Chemotherapie erhielten, unterlagen einem 21,6-fach erhöhten Risiko für ein Auftreten von ON in der GK-MRT ( $p=0,003)$. Der Verlauf der ON war sowohl unter einer Therapie aufgrund der Läsionen als auch ohne Behandlung bei 12 der betrachteten 15 Patienten (80 \%) regredient.

Schlussfolgerung: GK-MRT-Untersuchungen dienen der Detektion von ON bei pädiatrischen Patienten mit onkologischen oder hämatologischen Grunderkrankungen. Dabei ist vor allem die Darstellung des gesamten Körpers mittels der T1-SPACE-Sequenz hilfreich. Die Läsionen treten vor allem in der gewichtstragenden unteren Extremität auf. Auf die Risikofaktoren Alter $>10$ Jahre bei der Diagnose der Grunderkrankung bzw. bei der ersten SZT, weibliches Geschlecht und die verabreichte Steroidmedikation sollte geachtet werden. Zukünftig sollte der Nutzen einer spezifischen Therapie hinsichtlich aufgetretener ON genauer analysiert werden und Zeichen in der MRT gefunden werden, die frühe ON identifizieren und somit die Häufigkeit dieser Spätkomplikation reduzieren können. 


\section{Einleitung}

Mit steigenden Überlebensraten bei onkologischen und hämatologischen Erkrankungen im Kindes- und Jugendalter steigt die Gefahr der Entwicklung von Spätkomplikationen, auf welche ein besonderes Augenmerk gelegt werden sollte, da sie zumindest die Lebensqualität nach überstandener Tumorerkrankung deutlich beeinträchtigen können. Eine skelettale Komplikation ist die Entstehung von Osteonekrosen (ON), welche nicht nur im Rahmen antileukämischer Therapie bzw. als Spätfolge nach stattgehabter Behandlung sondern auch nach Stammzelltransplantation auftreten können. Eine frühe Identifikation der ON sowie deren Behandlung sind wichtig für eine angemessene Lebensqualität (Leung et al. 2007, Kaste et al. 2011, Miettunen et al. 2012, Duncan et al. 2015). Bei der Diagnostik der Knochennekrosen können weder die klinische Symptomatik noch Laborparameter zuverlässige Auskunft geben. Diagnostikum der Wahl ist die Bildgebung mittels Magnetresonanztomographie (MRT) (von Stillfried und Weber 2014). Aufgrund der häufig multifokalen Lokalisation der Läsionen ist es hilfreich, den gesamten Körper mittels einer Ganzkörper-Magnetresonanztomographie (GK-MRT) in einer Untersuchung abzubilden.

\subsection{Osteonekrosen}

\subsubsection{Pathogenese und Ursachen der Osteonekrosenentstehung}

Die Grundlage der Entstehung von Osteonekrosen (ON) liegt in einer unzureichenden vaskulären Versorgung des Knochens, wodurch es zur Ischämie und nachfolgend zum Absterben des Knochengewebes - der Nekrose - kommt (Assouline-Dayan et al. 2002, von Stillfried und Weber 2014). Durch traumatische Läsionen von Blutgefäßen, intraluminale Gefäßverschlüsse durch Thrombembolie oder Kompression der Gefäße von außen kann die Blutversorgung des Knochens unterbrochen werden. Traumata können durch die mechanische Gefäßverletzung direkt zu einer Unterbrechung der Blutversorgung, z.B. aufgrund einer dislozierten Fraktur mit Einbeziehung der Blutgefäße, führen (Assouline-Dayan et al. 2002, Link 2006).

Hinsichtlich der atraumatischen Ursachen besteht deutlich mehr Unklarheit in der Pathogenese. Assouline-Dayan et al. fassen mehrere mögliche Theorien bezüglich des Auftretens einer ON zusammen (Assouline-Dayan et al. 2002). Neben der 
beschriebenen vaskulären Okklusion mit nachfolgender Ischämie, z.B. bei Artherosklerose, spielen auch intravaskuläre Koagulationsveränderungen, wie z.B. Thrombophilie oder Hypofibrinolyse, sowie sekundäre intramedulläre Hämorrhagien bei Arteriopathie eine Rolle. Weitere mögliche pathogenetische Faktoren sind ein erhöhter intrakortikaler Druck, der sekundäre arterielle Verschluss im Rahmen von Heilungsprozessen oder mechanischer Stress auf den Knochen, der vor allem in der gewichtstragenden unteren Extremität ursächlich sein könnte. Als weitere Ursachen der Entstehung von ON werden die Inhibition der Angiogenese, ein erhöhtes Apolipoprotein B bzw. A1 und der primäre Zelltod u.a. durch Steroide oder Alkohol genannt (Assouline-Dayan et al. 2002, Link 2006).

Die am häufigsten vermutete Ursache für die Entstehung von ON im Rahmen onkologischer Erkrankungen ist die Hochdosischemotherapie mit Kortikosteroiden (Ley et al. 2009). Zur Erklärung der Pathogenese zwischen der Steroidgabe und dem vermehrten Auftreten der ON existieren mehrere Hypothesen. Neben der direkten Toxizität der Steroide auf die Osteozyten, wodurch es zur Apoptose und nachfolgender Nekrose kommt, wird ebenfalls eine vermehrte Schädigung der venösen Drainage des Knochenmarks diskutiert (Geczova et al. 2015). Eine weitere Erklärung ist die durch den Einfluss der Glukokortikoide gesteigerte Proliferation und Hypertrophie der Lipozyten im Knochenmark, wodurch der intramedulläre Druck steigt. Der Blutfluss wird reduziert und es kommt zur Ischämie im Knochen. Dies wird durch das pubertäre Knochenwachstum mit Schluss der Epiphysenfuge verstärkt (Mattano et al. 2012). Aufgrund der zunehmenden Fettzellmasse unter der Steroidmedikation wird ebenfalls eine Fettembolie mit sekundärer thrombotischer Ischämie als Grundlage der vermehrten Entwicklung von ON erörtert (Niinimaki et al. 2008).

Auch umweltbedingte Faktoren wie Alkohol oder Rauchen und metabolische Ursachen, z.B. Diabetes mellitus, Morbus Gaucher, Schwangerschaft, sowie gastrointestinale Einflüsse wie die Pankreatitis spielen eine mögliche Rolle bei der Entstehung von ON. Neben dem Einfluss von Steroiden auf die Nekrosenentwicklung, welche auch nach Nierentransplantationen zur Immunsuppression verabreicht werden, gilt als weiterer möglicher iatrogener Grund die Radiotherapie, z.B. beim Lymphom (Saini und Saifuddin 2004). Zusätzlich zu diesen genannten möglichen Ursachen halten verschiedene Autoren auch Hämoglobinopathien, z.B. Sichelzellanämie, Polycythämie sowie Vaskulitiden und 
Kollagenosen als relevant in der Entstehung von ON (Saini und Saifuddin 2004, Link 2006). Idiopathische Fälle, bei denen keine Ursache gefunden werden kann, sind zudem beschrieben (Roth et al. 2015).

Zusammengefasst ist die Entstehung der ON multifaktoriell bedingt und wie eingangs beschrieben immer noch nicht vollständig geklärt (Assouline-Dayan et al. 2002). Die Dauer der Erkrankung hängt wesentlich von der Einsprossung neuer Blutgefäße und somit von Umbauvorgängen im Knochen mit Abbau, Resorption und Aufbau neuer Knochenbälkchen ab. Ziel der Therapie von ON ist es, die unzureichende vaskuläre Versorgung wieder zu stabilisieren (von Stillfried und Weber 2014).

\subsubsection{Inzidenz, Symptomatik und Lokalisationen der Osteonekrosen}

Die Inzidenz der ON bei Kindern und Jugendlichen mit malignen Grunderkrankungen variiert zwischen 1,4 \% bis maximal 73,9 \% (Lackner et al. 2005, Ippolito et al. 2016). Die Ausprägung der Symptomatik bei ON reicht von kompletter Schmerzfreiheit über den Ruheschmerz hin zur schmerzbedingt vollständigen Bewegungseinschränkung. Sind die kleinen Gelenke betroffen, findet sich eher eine druckschmerzhafte Schwellung, bei größeren Gelenken Bewegungsschmerz mit Funktionseinschränkung. Ebenfalls kann der Schmerz in andere Regionen fortgeleitet werden, so kann beispielsweise bei einer ON im Femurkopf der Schmerz in das Kniegelenk projiziert werden (von Stillfried und Weber 2014).

ON sind am häufigsten in der gewichtstragenden unteren Extremität lokalisiert, dort vor allem in den langen Röhrenknochen Femur und Tibia. In der oberen Extremität ist der Humerus am häufigsten betroffen (Miettunen et al. 2012). Andere Lokalisationen sind das Sprunggelenk, das Becken, die Wirbelsäule sowie Ellenbogen- und Handgelenk (Mattano et al. 2000, Saini und Saifuddin 2004, Li et al. 2014). Sharma et al. finden ON bei Patienten nach Stammzelltransplantation häufiger im Knie- als im Hüftgelenk, dagegen aber schwerwiegender bezüglich der Symptomatik in der Hüfte aufgrund einer höheren epiphysären Beteiligung von > 30 \% der Gelenkfläche (Sharma et al. 2012).

\subsubsection{Risikofaktoren für die Entwicklung von Osteonekrosen}

Als Hauptrisikofaktor für das Auftreten von ON wird von vielen Autoren ein Alter des Patienten von über zehn Jahren bei Diagnosestellung der Grunderkrankung bzw. bei der Stammzelltransplantation genannt (Kawedia et al. 2011, Sharma et al. 2012, Girard et al. 2013). Bei den älteren Kindern und Jugendlichen finden sich vermehrt 
Nekrosen, während Knochenödeme als deren Vorstufe häufiger bei jüngeren Patienten auftreten (Scherer et al. 2001).

Hinsichtlich der Geschlechtspräferenz sind die Ergebnisse nicht einheitlich. Es wird sowohl bei Patienten mit als auch ohne Stammzelltransplantation (SZT) ein höheres Risiko für das weibliche Geschlecht beschrieben. Auch findet bei Frauen die Entwicklung der Läsionen früher statt (Mattano et al. 2012, Li et al. 2014). Im Gegensatz dazu sehen mehrere Autoren dahingehend keinen Zusammenhang (Girard et al. 2013, Kaste et al. 2015).

Weitere in Studien analysierte Risikofaktoren sind zum einen die initiale Leukozytenzahl, welche keine Korrelation zur vermehrten Entwicklung von ON zeigt (Engelbrecht et al. 2000), sowie der Body-Mass-Index und die ethnische Herkunft. Niinimäki et al. beschreiben bei Patienten mit erhöhtem Body-Mass-Index ein erhöhtes ON-Risiko (Niinimaki et al. 2007), andere Autoren finden allerdings keinen Zusammenhang (Kaste et al. 2015). Mattano et al. stellen bei Patienten im Alter von über zehn Jahren ein höheres Risiko für das Auftreten von $\mathrm{ON}$ bei Menschen mit weißer Hautfarbe im Vergleich zu Personen mit schwarzer Hautfarbe fest (Mattano et al. 2000). Dagegen stehen mehrere Studien, die hinsichtlich der genetischen Herkunft und einer häufigeren Entwicklung von ON keine Korrelation finden (Sharma et al. 2012).

Weiterhin gehen Therapieschemata, bei denen die Applikation von Steroiden enthalten ist, mit einem erhöhten Risiko für die Entwicklung von ON einher (Girard et al. 2013). Im Vergleich zwischen Prednisolon und Dexamethason als Ursache für die Nekrosenentstehung gilt letzteres als toxischer auf den Knochen wirkend (Kunstreich et al. 2016). Auch spiegelt sich die höhere Steroiddosis als Risikofaktor in der Gruppe der Stammzelltransplantierten wider, dort vor allem die Verabreichung nach der Transplantation (Faraci et al. 2006).

In Betrachtung der onkologischen Patienten nach erfolgreicher SZT gilt die Transplantation an sich im Vergleich zu Patienten ohne SZT als Risikofaktor mit häufigerem und früherem Auftreten von ON (Girard et al. 2013, Kunstreich et al. 2016). Hinsichtlich des Spendertyps zeigt sich ein erhöhtes Risiko bei Patienten mit allogener SZT im Gegensatz zu Patienten mit autologer SZT (Girard et al. 2013). Bei mehreren Stammzellgaben besteht kein zusätzliches Risiko (Karimova et al. 2007, McAvoy et al. 2010). Hinsichtlich des Einflusses der Konditionierung vor der SZT gibt es keine einheitlichen Ergebnisse, ob die Ganzkörperbestrahlung mit einem erhöhten 
ON-Risiko einhergeht (Faraci et al. 2006) oder keine Korrelation besteht (Campbell et al. 2009). Diese Problematik findet sich ebenfalls bei Betrachtung der Graftversus-Host-Disease (GvHD) und ihrer Behandlung. Die Auswertungen reichen von keinem Zusammenhang (Sharma et al. 2012) über ein vermehrtes Vorkommen von ON bei vor allem chronischer GvHD (Faraci et al. 2006). Das erhöhte Risiko bei der GvHD geht vermutlich mit den verabreichten Steroiden zur Therapie dieser Erkrankung einher.

Niinimäki et al. beschreiben ein vermehrtes Vorkommen von ON bei Kindern mit malignen Lymphomerkrankungen (Niinimaki et al. 2008). Dabei könnte laut Enrici et al. die in ihrer Studie angewandte supradiaphragmatische Bestrahlung im Rahmen des Morbus Hodgkin als Risikofaktor gelten (Enrici et al. 1998). Karimova et al. sehen beim Vergleich von Patienten mit akuter lymphatischer Leukämie (ALL), akuter myeloischer Leukämie (AML) und Non-Hodgkin-Lymphom (NHL) keine Korrelation zwischen der jeweiligen Grunderkrankung und einem erhöhten Risiko für das Auftreten von ON im kniegelenknahen Bereich (Karimova et al. 2010).

Gegenstand der aktuellen Forschung sind genetische Risikofaktoren für das Auftreten von Knochennekrosen. Tumornekrosefaktor- $\alpha$ ist der wichtigste Regulator der Apoptose. Polymorphismen dieses Faktors stehen in Korrelation mit einem vermehrten Auftreten von ON (Liu et al. 2015). Verschiedene Variationen von Einzelnukleotidpolymorphismen (Single Nucleotide Polymorphism - SNP) spielen auch in der Entstehung von ON eine Rolle, dabei vor allem jene im Glutamatrezeptor auf Chromosom 21 und dessen Signalweg (Kawedia et al. 2011, Karol et al. 2015). Manche genetische Risikofaktoren sind dabei altersspezifisch mit einer Altersgrenze von zehn Jahren. So beeinflussen Genvarianten, welche für die Differenzierung mesenchymaler Stammzellen zu z.B. Osteoblasten bedeutsam sind, das Risiko für ON bei Kindern mit ALL im Alter von unter 10 Jahren. Dagegen ist der Einfluss des Glutamatrezeptorsignalwegs auf die Pathogenese der ON bei diesen Kindern unabhängig vom Alter (Karol et al. 2016).

\subsubsection{Verlauf und Komplikationen der Osteonekrosen}

Roth et al. beschreiben in einer S3-Leitlinie den natürlichen Verlauf einer atraumatischen Femurkopfnekrose bei Erwachsenen als progredient, die unbehandelt innerhalb von zwei Jahren zu einer subchondralen Fraktur führen kann (Roth et al. 2015). Schlussendlich ist bei einem solchen Fall die Erhaltung des Gelenks nicht mehr möglich. Dies gilt vor allem bei beidseitigem Befall und bei 
anhaltender Noxe. Innerhalb von zwei Jahren nach initialem Auftreten von ON besteht ein erhöhtes Risiko für den Befall der Gegenseite, danach sinkt es. Bei diesen Aussagen findet sich starker Expertenkonsens (Roth et al. 2015).

Nekrosen mit progredienter Volumenausdehnung sind bereits initial größer als jene mit stationärem Verlauf und diese wiederum sind größer als Läsionen mit spontaner Rückbildung (Engelbrecht et al. 2000). So ist laut Körholz et al. eine Größe von > 900 $\mathrm{mm}^{2}$ signifikant mit einem erhöhten Risiko für die Progression der ON assoziiert (Korholz et al. 1998). Karimova et al. sehen eine Verbindung zwischen einer Femurkopfnekrose mit einer Größe von > 30 \% des Volumens der Epiphyse und einer schlechten Prognose mit vorzeitigem Sintern des Femurkopfes und nachfolgend frühzeitigem Gelenkverschleiß (Karimova et al. 2007). Entscheidende Einflussfaktoren auf den Verlauf der ON sind neben der Größe der Läsion auch die Lage im Knochen mit möglicher Gelenkflächenbeteiligung. Hinsichtlich der Lage der ON in den langen Röhrenknochen sind große, epiphysär gelegene Nekrosen mit möglicher Gelenkflächenbeteiligung eher mit einem Gelenkeinbruch und somit mit einem schlechteren Verlauf vergesellschaftet als Läsionen im Bereich der Meta- und Diaphyse (Engelbrecht et al. 2000). Laut Karimova et al. steigt das Risiko für einen knöchernen Einbruch vor allem dann, wenn über $50 \%$ der Gelenkfläche von der Läsion betroffen sind (Karimova et al. 2010).

Als Komplikation des Auftretens von ON bei Kindern und Jugendlichen gilt die eingeschränkte Gelenkfunktion mit eventuell nachfolgend notwendiger chirurgischer Intervention und somit verminderter Lebensqualität (Kaste et al. 2011). Girard et al. analysieren in ihrer Studie den Einfluss von symptomatischen ON bei Kindern auf die Lebensqualität im späteren Erwachsenenalter mit Hilfe des Fragebogens SF-36 (Girard et al. 2013). Das Vorhandensein von Nekrosen im Kindesalter wirkt sich negativ auf den zusammengefassten Score der betrachteten physischen Bereiche aus. Von den insgesamt 8 erfragten Dimensionen werden signifikante Verschlechterungen der körperlichen Funktionsfähigkeit, der körperlichen Rollenfunktion, des physischen Schmerzes und der allgemeinen Gesundheitswahrnehmung verzeichnet. Weitere Komplikationen, die die Lebensqualität negativ beeinflussen können, sind pathologische Frakturen, welche bei sinkender Knochendichte vermehrt auftreten. Vor allem nach Manifestation von ON bestätigen den Hoed et al. eine signifikante Verminderung der mineralisierten Knochensubstanz bei betroffenen Patienten, gemessen anhand der Knochendichte 
der lumbalen Wirbelsäule und des gesamten Skeletts (den Hoed et al. 2015). Des Weiteren untersuchen Girard et al. mit Hilfe des Gesundheitsfragebogens SF-36 auch die psychischen Auswirkungen von vorhandenen symptomatischen $\mathrm{ON}$ bei Kindern. Sie sehen keinen signifikanten Einfluss auf die mentale Lebensqualität im späteren Erwachsenenalter (Girard et al. 2013).

\subsubsection{Therapie der Osteonekrosen}

Die Therapie der ON ist bislang mit verschiedenen konservativen bis hin zu operativen Methoden nicht einheitlich und reicht von kurativen zu symptomatischen Maßnahmen bis zur Strategie der Beobachtung des Verlaufs ohne Therapie. Für jeden Patienten sollte in Abhängigkeit vom Ausmaß und der Verteilung der ON eine individuelle Auswahl in der Behandlung getroffen werden. Dabei ist vor allem eine Anpassung der Chemotherapie erforderlich, wenn im Rahmen dieser Steroide, welche als Risikofaktor für das Auftreten von ON gelten, verabreicht werden (te Winkel et al. 2011, den Hoed et al. 2015).

Zunächst wird die Schonung und Entlastung der betroffenen Extremität mit Gehstützen teils kombiniert mit medikamentöser Therapie, z.B. Calcium- und Vitamin-D-Substitution oder Gabe von nichtsteroidalen Antiphlogistika, empfohlen (Burger et al. 2005). Engelbrecht et al. machen auf die dann erforderliche Mehrbelastung der oberen Extremität bei Entlastung der Beine aufmerksam, was wiederum zu vermehrten $\mathrm{ON}$ in den Armen führen könnte (Engelbrecht et al. 2000). Ist die Wirbelsäule betroffen und besteht Sinterungsgefahr, so werden entsprechend Stützkorsette und andere Hilfsmittel verordnet. Ebenfalls sollte Physiotherapie durchgeführt werden, um die unzureichende Durchblutung als Hauptursache für die Entstehung der ON zu verbessern. Für Läsionen am Fußskelett werden Einlagen verschrieben (von Stillfried und Weber 2014). Allerdings bringt die alleinige konservative Therapie am Beispiel der Femurkopfnekrose laut Roth et al. keine anhaltende Beschwerdelinderung und das Aufhalten der Progression ist nicht möglich (Roth et al. 2015). Sie sollte aufgrund des geringen Benefit immer nur Teil eines Gesamtbehandlungskonzepts sein.

In frühen Stadien der ON gelten die Infusionstherapie mit Prostazyklinen, v.a. lloprost, sowie die Nutzung von Bisphosphonaten, z.B. Alendronat, Zoledronat oder Pamidronat, als Therapievarianten. Diese Medikamente werden zur Behandlung von Osteonekrosen im „off-label-use“ verwendet. Eine weitere Behandlungsmöglichkeit ist die hyperbare Sauerstofftherapie (Roth et al. 2015). Zwar führt sie zur 
Verminderung des Schmerzes bei gering ausgeprägten Nekrosen, wird aber aufgrund mangelnder Evidenz des Therapieerfolges ebenfalls wie die Anwendung von z.B. Antikoagulantien, Statinen, Ultraschall- und Stoßwellentherapie oder Elektrostimulation zur Therapie von ON nicht empfohlen (Roth et al. 2015).

Ziel einer operativen Intervention ist die verbesserte vaskuläre Versorgung in der Nekrosezone sowie das Erhalten der normalen Gelenkfunktion (von Stillfried und Weber 2014). Es werden unter anderem Anbohrungen mit und ohne autologer oder allogener Knochentransplantation durchgeführt (Assouline-Dayan et al. 2002). Weitere chirurgische Eingriffe sind Osteotomien, um das nekrotische Knochensegment aus der gewichtstragenden Zone herauszubringen und somit den mechanischen Stress zu vermindern. Bei sehr starkem Progress der ON, z.B. bei massivem Gelenkeinbruch, ist der Ersatz durch eine Totalendoprothese die letzte Behandlungsmöglichkeit (Assouline-Dayan et al. 2002). Eine operative Behandlung ist bei bis zu $50 \%$ der betroffenen Patienten erforderlich (Socie et al. 1997, McAvoy et al. 2010).

\subsection{Bildgebung von Osteonekrosen}

\subsubsection{Mögliche MRT-Sequenzen zur Darstellung von Osteonekrosen}

Morphologisches Hauptmerkmal von ON im MRT-Schnittbild ist die girlandenartige Konfiguration, die vor allem nach erfolgter Kontrastmittelapplikation in T1-wichtenden Sequenzen mit Fettgewebsunterdrückung deutlich wird (Beer et al. 2008). Hinsichtlich der Signalintensität ergeben sich je nach verwendeter Sequenz Unterschiede. In der T1w-Sequenz findet sich eine zum umgebenden Knochenmark iso- bis hypointense Läsion mit hypointenser Umrandung, welche histologisch betrachtet die reaktive Randzone widerspiegelt. Typisch für die Darstellung von Knochennekrosen in der T2-gewichteten Sequenz ist das sogenannte "double line sign“. Dabei umgibt den hyperintensen Rand um die signalarme Läsion ein parallel zu diesem verlaufender Rahmen mit geringer Signalintensität. Die hyperintense Zone entspricht dabei reparativem Granulationsgewebe (Mitchell et al. 1987, Zurlo 1999, Beer et al. 2008). Mit Hilfe von Kontrastmittel und Fettunterdrückung lässt sich die Umrandung der Läsion in der T1-Wichtung aufgrund vermehrter Kontrastmittelaufnahme signalreich darstellen (Beer et al. 2008). Vorhandene 
Restperfusion und Revaskularisation können durch die Kontrastmittelapplikation ebenfalls nachgewiesen werden (Link 2006).

Neben diesen Sequenzen dienen als weitere Möglichkeiten für die Nekrosendarstellung die Short Tau Inversion Recovery (STIR)- und die Diffusion Weighted Imaging (DWI)-Sequenz (Kubo et al. 2015, Ozel et al. 2016). Im Gegensatz zu T2-gewichteten Aufnahmen wird bei der STIR-Sequenz durch den vorgeschalteten Inversionspuls eine homogenere und stabilere Fettunterdrückung erreicht mit resultierend gutem Kontrastverhältnis zwischen der ON und dem Knochenmark (Goo 2015, Nievelstein und Littooij 2015). Frühe trophische Veränderungen lassen sich in der STIR-Sequenz als hyperintens inhomogene fleckige Läsionen abgrenzen (Kubo et al. 2015). Sie ist wie die diffusionswichtende Sequenz zur Beurteilung eines Ödems in der MRT-Bildgebung anerkannt. Die DWISequenz beruht auf der Diffusion von Wasserstoffprotonen in Geweben. Sie wird überwiegend in der Schlaganfalldiagnostik zur Differenzierung von vasogenem und zytotoxischem Ödem eingesetzt. In der Ganzkörperbildgebung findet sie vorrangig zur Detektion von aktiven Läsionen z.B. in der Onkologie oder Rheumatologie Anwendung. Unter Einsatz verschiedener Diffusionswerte können Diffusionskoeffizienten quantifiziert werden (Apparent Diffusion Coefficient - ADC). Läsionen mit einem erhöhten ADC entsprechen dabei Arealen mit erhöhter Diffusivität, erhöhtem Wassergehalt bzw. einem eher vasogenem Ödem, während Läsionen mit ADC-Minderung dicht gepackte Regionen mit erhöhter Zellularität charakterisieren (Reimer et al. 2003). Ebenfalls dienen die ADC-Werte zur differentialdiagnostischen Beurteilung von ON. Ozel et al. ermittelten signifikant höhere ADC-Werte in von Nekrosen betroffenen Regionen als im gesunden Knochen (Ozel et al. 2016). Dieser Befund ist besonders in fortgeschrittenen Stadien der ON deutlich und hilfreich bei der Abgrenzung gegenüber malignen Läsionen, die eine Minderung des ADC zeigen. Inwiefern ein zusätzlicher Nutzen der Beurteilung quantitativer Diffusionswerte im Vergleich zur konventionellen MRT in der Aussagekraft bezüglich der Prognose der ON besteht, kann derzeit noch nicht abschließend beantwortet werden.

\subsubsection{Nutzen der MRT zur Detektion von Osteonekrosen}

Beim Nachweis von Knochenläsionen erreicht die GK-MRT eine Sensitivität von bis zu 100 \% (Krohmer et al. 2010). Vor allem mit den Turbo Spin Echo (TSE)Sequenzen ist es möglich, rotes, hämatopoetisches Knochenmark von gelbem 
Fettknochenmark zu differenzieren. Pathologische Läsionen im Knochenmark stellen sich in der T1-Wichtung zumeist signalarm und in der T2w-Sequenz hyperintens dar (Nievelstein und Littooij 2015). Ein großer Vorteil der MRT ist der Nachweis von symptomatischen als auch klinisch stummen ON (Beer et al. 2008). Von mehreren Autoren wird die MRT als die sensitivste Methode zur Nekrosedarstellung im Vergleich zu anderen Bildgebungen und somit als Methode der Wahl beschrieben (Link 2006). Nicht nur zum Nachweis von sehr frühen Veränderungen im Knochenmark sondern auch für die Verlaufsbeurteilung ist die MRT hilfreich (Engelbrecht et al. 2000, Saini und Saifuddin 2004). Diese sollte, wenn kein GK-MRT durchgeführt wird, im Bereich der betroffenen Lokalisation möglichst bilateral erfolgen (Jaramillo 2009).

Röntgenologisch bleiben frühe ON Wochen bis Monate nach ihrem Beginn negativ und werden erst als späte Veränderung unter anderem durch das sogenannte „crescent sign“ als Zeichen für eine subchondrale Fraktur entdeckt (Assouline-Dayan et al. 2002, Link 2006).

Anhand der Röntgen- bzw. MRT-Befunde wird eine atraumatische Femurkopfnekrose nach der ARCO-Klassifikation (Association Research Circulation Osseous) in fünf Stadien eingeteilt (Tabelle 1) (von Stillfried und Weber 2014).

Tabelle 1: ARCO-Klassifikation der atraumatischen Femurkopfnekrose (von Stillfried und Weber 2014)

\begin{tabular}{|c|c|c|c|}
\hline ARCO & Stadium & Röntgen & MRT \\
\hline 0 & Initialstadium & Negativ & Negativ \\
\hline I & $\begin{array}{c}\text { Reversibles } \\
\text { Frühstadium }\end{array}$ & Negativ & Ödem ohne Demarkation \\
\hline II & Irreversibles & Sklerotischer Randsaum & $\begin{array}{c}\text { Nekrose, } \\
\text { Doppellinienzeichen } \\
\text { („,double line sign“) }\end{array}$ \\
\hline III & Knocheninsuffizienz & Subchondrale Fraktur & $\begin{array}{c}\text { Subchondrale Fraktur } \\
\text { („crescent sign“) }\end{array}$ \\
\hline IV & Spätstadium & $\begin{array}{c}\text { Sekundär arthrotische } \\
\text { Veränderungen }\end{array}$ & $\begin{array}{c}\text { Einbruch der Nekrose, } \\
\text { sekundär arthrotische } \\
\text { Veränderungen }\end{array}$ \\
\hline
\end{tabular}

In der Szintigraphie zeigt sich aufgrund der vorliegenden Hyperämie in der Umgebung der Nekrosen eine gesteigerte Isotopenaufnahmen (Mitchell et al. 1987). Jaramillo merkt an, dass die Szintigraphie bei Unzugänglichkeit zur MRT sinnvoll in 
der Detektion von ON ist (Jaramillo 2009). Mit Hilfe der Computertomographie (CT) kann das Stadium einer ON nicht beurteilt werden. Erst fortgeschrittene ON sind im Rahmen von Gelenkeinbrüchen oder Frakturen sichtbar. Die Sonographie spielt bei der Beurteilung von Knochenmarkprozessen wie den ON keine Rolle. Ebenfalls wird eine Angiographie im Rahmen der Diagnostik von ON nicht empfohlen (AssoulineDayan et al. 2002).

\subsubsection{Differentialdiagnosen der Osteonekrosen in der MRT}

ON können bei nicht klaren charakteristischen bildmorphologischen Kriterien in der MRT schwer von radiologisch ähnlichen Diagnosen abgrenzbar sein. Dabei handelt es sich um Metastasen, Rezidive einer Leukämie oder unspezifische transiente Signalveränderungen (Kaste et al. 2011). Eine davon ist das Knochenmarködemsyndrom oder auch transiente migratorische Osteoporose genannt, welche zu Signalerhöhungen in den T2-wichtenden fettgesättigten Sequenzen führt (Saini und Saifuddin 2004). Weiterhin sind differentialdiagnostisch entzündliche Knochenveränderungen, z.B. Osteitis, Osteomyelitis, Markraumphlegmone oder Abszess, sowie solide Knochentumore wie das maligne Osteosarkom oder das benigne Chondroblastom abzugrenzen (Beer et al. 2008). Im Gegensatz zu den Metastasen zeigen fortgeschrittene ON im Schnittbild die charakteristische girlandenförmige Umrandung der Läsion (Goo et al. 2005).

\subsection{Ganzkörper-Magnetresonanztomographie im Kindesalter}

\subsubsection{Entwicklung der GK-MRT und Möglichkeiten in der Pädiatrie}

Durch Entwicklungen in der Scanner-, Spulen- und Sequenztechnologie konnte sich die GK-MRT in den letzten Jahren zunehmend etablieren. Waren zunächst enorm lange Untersuchungszeiten limitierend, konnte insbesondere auch durch die parallele Bildgebung die Messzeit für die Ganzkörperdarstellung vom Kopf bis zu den Füßen deutlich verkürzt werden. Je nach Fragestellungen sind Messprotokolle von 30 bis 60 Minuten möglich. Einen relativ großen Zeitfaktor nimmt aber die Lagerung des Patienten in der Kopfspule sowie das Anbringen von flexiblen Spulenelementen über dem gesamten Körper und die anschließende Planung der Untersuchung ein. Aufgrund der fehlenden Strahlenbelastung ist die GK-MRT im Vergleich zu anderen Ganzkörperverfahren wie der CT, der Positronenemissionstomographie (PET) oder der Kombination aus PET und CT (PET-CT) insbesondere für das Kindesalter eine 
interessante Methode bei verschiedenen Fragestellungen wie z.B. onkologischen Erkrankungen, Syndromen oder Fokussuchen. Mit einem automatisch verschiebbaren Untersuchungstisch, der eine Umlagerung des Patienten unnötig macht, werden die Daten im Isocenter des Magneten aufgenommen, wo die Magnetfeld-Inhomogenität minimal ist. Auch gelten Körperspulen, die den gesamten Körper bedecken, sowie schnellere 3D-Sequenzen als Fortschritt, um die Messzeit bei einer Ganzkörper-Untersuchung zu verkürzen und die Bildqualität zu erhöhen. Mit Hilfe entsprechender Software wird die Aufnahme sofort zu einem Bild verarbeitet, auf dem der gesamte Körper dargestellt wird (Darge et al. 2008, Goo 2015).

\subsubsection{Vor- und Nachteile der GK-MRT}

Der größte Vorteil der MRT liegt darin, dass keine ionisierende Strahlung eingesetzt werden muss, sondern die Bilderzeugung auf der Grundlage von statischen und wechselnden Magnetfeldern beruht. Schädigende Auswirkungen der Magnetfelder sind unter Berücksichtigung der Grenzwerte ihrer Anwendung nicht zu erwarten. Die Untersuchung kann theoretisch beliebig oft wiederholt werden. Dies ist für Verlaufskontrollen in der Pädiatrie vor allem bei Kindern mit onkologischen Erkrankungen von großer Bedeutung (Krohmer et al. 2010, Goo 2015). Neben der knöchernen Beurteilung erfolgt im Rahmen derselben Untersuchung auch die Ausbreitungsdiagnostik in Weichteilen und Organen, um so das gesamte systemische Ausmaß einer Erkrankung zu detektieren und dadurch eventuell auf weitere Bildgebungen verzichten zu können. Schlussfolgernd kann mit der hohen diagnostischen Aussagekraft der GK-MRT die Therapie schneller erfolgen, da nicht viele einzelne Teilkörperuntersuchungen durchgeführt werden müssen (Schaefer und Kramer 2011).

Vorteile gegenüber der Ganzkörperbildgebung mittels CT bzw. PET-CT liegen im hohen Weichteilkontrast, der angesprochenen Abwesenheit der ionisierenden Strahlung und auch in der Möglichkeit zur nichtinvasiven Angiographie (Gaa 2006). Im Gegensatz zu nuklearmedizinischen Ganzkörperuntersuchungen wie z.B. der Skelettszintigraphie besteht bei der GK-MRT eine hohe räumliche Auflösung (Laffan et al. 2004). Nach Schaefer und Kramer ist eine Knochenszintigraphie nicht mehr erforderlich, wenn eine GK-MRT-Untersuchung vorliegt (Schaefer und Kramer 2011). Trotz der vielen Weiterentwicklungen gilt die GK-MRT immer noch als eine Untersuchungsmethode mit langer Messzeit, welche vor allem bei jungen oder 
unkooperativen Kindern mit Hilfe von Narkose, Sedierung oder Immobilisierung anderer Art überbrückt werden muss. Verschiedene Autoren empfehlen diese bei Mädchen und Jungen bis zum siebenten bzw. achten Lebensjahr, um bewegungsbedingte Artefakte zu minimieren (Ley et al. 2009, Schaefer und Kramer 2011). Nach Erfahrungen an der Kinderklinik Jena können GK-MRT-Untersuchungen auch bei Kindern jüngeren Alters ohne Narkose oder Sedierung erfolgen, wenn eine entsprechende Vorbereitung des Kleinkindes vorgenommen werden kann. Dies ist aber keine Selbstverständlichkeit und nicht in jedem Falle möglich. Eine Untersuchungsdauer von 50-60 min für die gesamte GK-MRT-Aufnahme sollte generell nicht überschritten werden (Ley et al. 2009, Goo 2015).

Grundsätzlich ist die MRT-Untersuchung gesundheitlich unschädlich, allerdings sind erhebliche Gefahren durch Unachtsamkeit oder falsche Anwendung möglich. Durch die Anziehung ferromagnetischer Objekte können Personen unter Umständen tödlich getroffen werden oder es verlagern sich ferromagnetische Implantate im Körper. Für Patienten mit Herzschrittmachern, Neurostimulatoren, Insulinpumpen und Ableitungsventilen wurde eine Grenze für die magnetische Flussdichte in der MRT von 0,5 mT bestimmt. Wichtig ist ebenfalls das Tragen eines Gehörschutzes, um Beeinträchtigungen des Gehörs infolge der erheblichen Lärmbelastung im Rahmen der MRT vorzubeugen. Weiterhin kann bei entsprechend hoher Magnetfeldflussdichte eine reversible periphere Nervenstimulation auftreten. Die Erwärmung beim Einsatz der MRT im klinischen Bereich und unter Berücksichtigung der Grenzwerte ist unbedenklich und liegt bei maximal $1^{\circ} \mathrm{C}$. Im Extremfall können lokale Verbrennungen an Haut-Haut-Kontakten oder an Stellen mit Tätowierungen auftreten (Möller 2006). Beim Einsatz von gadoliniumhaltigen MR-Kontrastmitteln sind bestimmte Risiken zu bedenken, so ist eine ausreichende Nierenfunktion notwendig, damit das MR-Kontrastmittel wieder ausgeschieden werden kann. Zudem sind im Kindesalter möglichst die stabileren zyklischen MR-Kontrastmittel einzusetzen, um den Anteil von im Körper abgelagertem Gadolinium beispielweise im Knochen, aber auch in der Haut, der Leber und im Gehirn zu reduzieren (Klee et al. 2017).

Aufgrund der hohen Zahl an aufgenommenen Bildern einer GK-MRT-Untersuchung (nach Erfahrungen in der Kinderklinik Jena bis über 4000 Bilder pro Untersuchung) ist die Befundung sehr aufwändig und erfordert eine hohe Aufmerksamkeit des Kinder-/Radiologen. Dies gilt insbesondere für Verlaufskontrollen, bei denen die 
Akquisition der Bilddaten unbedingt in gleicher Art und Weise erfolgen muss. Bei Screeninguntersuchungen können aufgrund der Vielzahl der Bilder kleinere Befunde untergehen, sodass diese Methode im Rahmen eines "Gesundheits-Checks" nur eingeschränkt empfohlen werden kann. Vor allem pathologische Veränderungen mit gleicher Signalintensität wie das sie physiologisch umgebende Gewebe sind leichter zu übersehen (Schaefer und Kramer 2011). Generell gibt es in der GK-MRT auch Regionen, die nur inkomplett bzw. sehr schwer beurteilt werden können wie beispielsweise die Rippen und die Wachstumszonen bei Kindern.

\subsubsection{Konzepte und technische Aspekte zur Durchführung einer GK-MRT}

Die Patienten werden in aller Regel in Rückenlage mit seitlich an den Körper angelegten supinierten Armen beginnend am Kopf untersucht (Ley et al. 2009). Ein Problem stellen Patienten mit kräftigem Körperbau dar, bei denen Schultern und Ellenbogengelenke, welche an der Körperseite liegen, nicht mehr im Messfeld enthalten sind (Gaa 2006). Für eine erweiterte Darstellung der Arme können diese über dem Kopf platziert werden, wobei sich allerdings die Dauer der Untersuchung verlängert (Darge et al. 2008). Als Kompromiss können die Hände bei der GK-MRT auf die Oberschenkel bzw. in den Schoß - ohne sie zu verschränken - gelegt werden, sodass die Ellenbogen näher an den Körper gelangen und somit die obere Extremität vollständig abgebildet werden kann.

Eine fortlaufende Ganzkörper-Untersuchung ist möglich, wenn der gesamte Körper mit Spulen (Phased-Array-Spule) bedeckt ist. Mit der Körperspule alleine, welche meist im Magneten integriert ist, wird nur ein geringes Signal-zu-Rausch-Verhältnis und eine niedrige räumliche Auflösung erreicht. Ein verbessertes Signal-zu-RauschVerhältnis und eine höhere Auflösung erzielt im Gegensatz dazu die Verwendung der genannten Phased-Array-Spule bestehend aus mehreren Elementen, welche überlappend auf dem gesamten Körper verteilt werden. Die einzelnen Aufnahmen überschneiden sich 3 - $5 \mathrm{~cm}$ für eine nahtlose GK-MRT. Mit diesen Oberflächenspulen besteht zudem die Möglichkeit der parallelen Bildgebung, mit deren Hilfe sich die Untersuchungszeit deutlich verkürzt. Vor allem bei funktioneller Bildgebung wie der diffusionsgewichteten Aufnahme ist die Anwendung der PhasedArray-Spule von Vorteil (Ley et al. 2009, Chavhan und Babyn 2011, Goo 2015, Mohan et al. 2015, Nievelstein und Littooij 2015).

Als Standardorientierung werden in der GK-MRT die Sequenzen in koronarer Ebene angefertigt, da somit große Teile des Körpers übersichtlich erfasst werden können. 
Zusätzlich kann eine sagittale Ebene für die Darstellung der Wirbelsäule nötig sein. Für die ZNS-Darstellung im Rahmen der GK-MRT werden axial orientierte Sequenzen (z.B. FLAIR - Fluid Attenuated Inversion Recovery) verwendet. Die bereits erwähnte Diffusionswichtung wird unter Verwendung verschiedener b-Werte ebenfalls in axialer Orientierung akquiriert und dient als zweite Ebene in der GKMRT. Im Gegensatz zum 2D-Scan-Modus für die verschiedenen Sequenzen besitzt der neu entwickelte 3D-Scan-Modus eine höhere räumliche Auflösung und die Möglichkeit der multiplanaren Rekonstruktion in andere Schichtorientierungen. Die Aufnahmeeffizienz und Bildqualität steigt (Darge et al. 2008, Goo 2015).

Um bewegungsbedingte Artefakte durch Atmung und Darmperistaltik zu vermindern, werden zum einen respiratorische Kompensations- bzw. Triggertechniken benutzt, bei denen sich jedoch die Untersuchungszeit verlängert, und zum anderen antiperistaltische Mittel verabreicht. Häufig müssen Sequenzen, welche die Lungenbzw. Abdominalregion abbilden, in Atemanhaltetechnik durchgeführt werden. Andere Möglichkeiten sind die Verwendung eines Atemgurtes, der die Atemexkursionen des Abdomens zu Hilfe nimmt, um die Untersuchung entsprechend zu triggern oder die Nutzung von Navigator-Techniken, bei denen die Sequenz durch die Bewegung des Zwerchfells gesteuert wird. Zur Unterdrückung der Darmmotilität stehen Butylscopolamid oder Glucagon zur Verfügung (Gaa 2006, Chavhan und Babyn 2011, Mohan et al. 2015, Nievelstein und Littooij 2015).

\subsubsection{Indikationen für die GK-MRT-Bildgebung in der Pädiatrie}

Neben onkologischen Indikationen für die GK-MRT in der Pädiatrie finden sich auch zunehmend Anwendungsgebiete im nicht-onkologischen Bereich. Aufgrund der Abbildung des gesamten Körpers in einer Untersuchungseinheit liegt das Augenmerk vor allem auf systemischen Erkrankungen. Indikationen im onkologischen Bereich sind vor allem das Staging bei malignen Erkrankungen. Bei Kindern mit verschiedenen genetischen Erkrankungen, wie z.B. der Ataxia teleangiectatica, stellt das CT aufgrund der erhöhten Radiosensitivität eine Kontraindikation dar. Das gleiche gilt für Patienten mit Krebsprädispositionssyndromen, wie z.B. dem LiFraumeni-Syndrom (Chavhan und Babyn 2011). Bei der Neurofibromatose Typ I oder der Langerhans-Zell-Histiozytose steht die Multifokalität von Weichteil- und Skelettläsionen im Vordergrund der bildgebenden Diagnostik. Mit Hilfe der GK-MRT werden sowohl die Knochenmarksbeteiligung, als auch die angrenzenden Weichteilläsionen sowie andere extraossäre Manifestationen in einer Aufnahme 
dargestellt. Fälle mit unklarem Primärtumor beim Cancer of unknown primary (CUP)Syndrom oder das Neuroblastom stellen weitere onkologische Indikationen für eine GK-MRT dar (Goo 2015, Nievelstein und Littooij 2015).

Im Rahmen der nicht-onkologischen Anwendungsgebiete stehen die Erkrankungen des rheumatischen Formenkreises und auch die Dermatomyositis im Vordergrund, da sie häufig eine multisystemische Ausbreitung aufweisen. Die GK-MRT ist für die chronisch rekurrierende multifokale Osteomyelitis in der Pädiatrie die Methode der Wahl, da sowohl symptomatische als auch klinisch stumme Manifestationen mit hoher Sensitivität aufgedeckt werden können. Weitere Anwendungen sind Fiebersyndrome wie das Fieber unklarer Ursache, um einen Infektionsfokus zu finden (Schaefer und Kramer 2011), und vaskuläre Malformationen, z.B. Hämangiome, Lymphangiome oder das Klippel-Trénaunay-Weber-Syndrom. Mehrere Autoren diskutieren den Einsatz der GK-MRT zur Bestimmung des Körperfettgehalts bei Adipositas, die Bildgebung als Alternative zur Autopsie als Post-mortem-Studie oder aber auch bei Verdacht auf körperliche Kindesmisshandlung (Gaa 2006, Darge et al. 2008, Ley et al. 2009). Nicht zuletzt dient die GK-MRT der Darstellung von symptomatischen als auch asymptomatischen ON, die als Komplikation der Hochdosischemotherapie bei unterschiedlichen malignen Grunderkrankungen pädiatrischer Patienten auftreten (Darge et al. 2008, Schaefer und Kramer 2011). 


\section{Ziele der Arbeit}

Osteonekrosen stellen eine Komplikation der Chemotherapie sowie der Stammzelltransplantation bei Kindern und Jugendlichen mit onkologischen sowie hämatologischen Grunderkrankungen dar. Die Magnetresonanztomographie gilt als Methode der Wahl zum Aufdecken solcher Läsionen im Knochen (Engelbrecht et al. 2000). Mit Hilfe der Ganzkörper-Aufnahme können in einem Untersuchungsgang der gesamte Körper aufgezeichnet und somit auch frühe, meist asymptomatische Nekrosen entdeckt werden (Beer et al. 2008, Miettunen et al. 2012).

In dieser retrospektiven Arbeit wurden GK-MRT-Aufnahmen bezüglich des Auftretens von Osteonekrosen bei pädiatrischen Patienten mit onkologischen und hämatologischen Grunderkrankungen ausgewertet. Dabei sollten folgende Fragen beantwortet werden:

1. Wie gut lassen sich Osteonekrosen in den einzelnen GK-MRT-Sequenzen qualitativ darstellen und welches Sequenzprotokoll kann zur alleinigen Beurteilung von Osteonekrosen empfohlen werden?

2. Wie häufig werden Osteonekrosen in der GK-MRT bei Kindern und Jugendlichen ohne und mit durchgeführter Stammzelltransplantation diagnostiziert?

3. Wie häufig sind in der GK-MRT nachgewiesene ON mit einer Schmerzsymptomatik verknüpft?

4. Welche Lokalisationen der Osteonekrosen im Körper werden mit Hilfe der GKMRT beobachtet?

5. Besteht ein Einfluss des Geschlechts oder des Alters bei der Diagnosestellung der Grunderkrankung bzw. bei der ersten Stammzelltransplantation auf den positiven Nachweis von Osteonekrosen in der GK-MRT?

6. Unterscheidet sich das Risiko des Auftretens der Osteonekrosen in der GKMRT je nach Art der verabreichten Steroide und Höhe der kumulativen Steroiddosis bei Patienten ohne Stammzelltransplantation?

7. Welchen Verlauf zeigen Osteonekrosen in der GK-MRT bei Patienten mit Morbus Hodgkin, die keine Stammzelltransplantation erhalten haben? Besteht ein Einfluss der verabreichten Osteonekrosentherapie? 


\section{Patienten und Methode}

\subsection{Auswahl und Einteilung der Patienten}

In diese retrospektive Studie wurden alle Kinder und Jugendlichen mit onkologischen und hämatologischen Grunderkrankungen eingeschlossen, die im Zeitraum vom 01.01.2008 bis 31.12.2016 mindestens eine GK-MRT am Universitätsklinikum Jena erhielten. Die Diagnose der jeweiligen Grunderkrankung wurde laborchemisch, histologisch oder radiologisch gesichert und durch die entsprechenden Referenzzentren bestätigt. Alle Patienten sind bzw. waren an die onkologischhämatologische Ambulanz der Kinderklinik Jena angeschlossen. Eine Einteilung der Patienten erfolgte anhand einer durchgeführten oder nicht erfolgten Behandlung mittels Stammzelltransplantation (SZT).

Folgende Einschlusskriterien wurden verwendet:

- Eine onkologische oder hämatologische Erkrankung war bereits diagnostiziert.

- Das Alter der Patienten ohne SZT betrug bei Diagnose der Grunderkrankung $\leq 20$ Jahre.

- Das Alter der Patienten mit SZT betrug bei der ersten Stammzelltransplantation $\leq 20$ Jahre: 4 Patienten wurden aufgrund ihres Alters ausgeschlossen.

- Es lag für jeden Patienten mindestens eine GK-MRT-Untersuchung vor, die am Universitätsklinikum Jena durchgeführt wurde. Dabei musste mindestens eine der folgenden vier Sequenzen vorliegen: T1-SPACE, T2-HASTE, T2HASTE-IR oder T1-SPACE-SPAIR mit Kontrastmittel.

Nach Ausschluss der vier Patienten mit einem Alter von über 20 Jahren bei der ersten SZT umfasste die Studie insgesamt 105 Patienten, wovon 62 Kinder und Jugendliche männlich (59 \%) und 43 weiblich (41\%) waren. Bei 63 Patienten (60 \%) erfolgte keine SZT und bei 42 Patienten (40 \%) wurde mindestens eine SZT im Verlauf ihrer Erkrankung vorgenommen. 

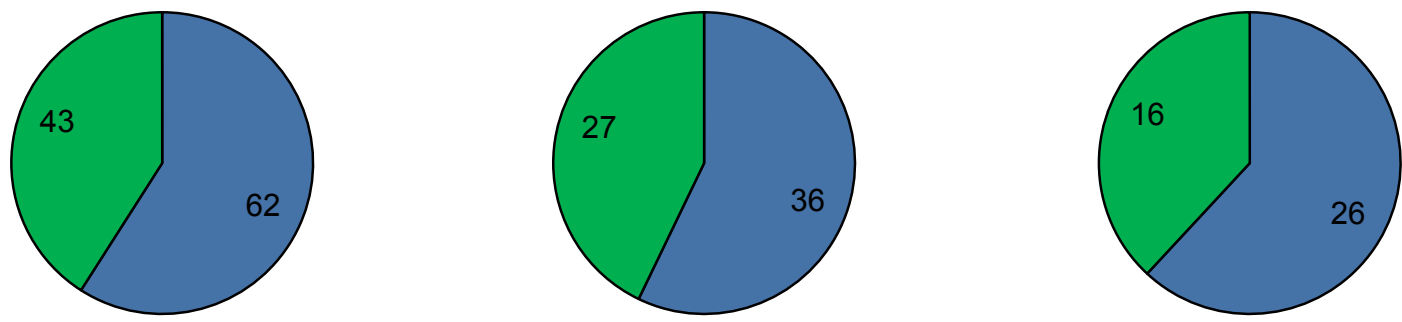

口männlich

$\square$ weiblich

Abbildung 1: Geschlechterverteilung in den einzelnen Patientengruppen

Die klinischen Daten der Patienten wurden retrospektiv aus den Akten der Klinik für Kinder- und Jugendmedizin sowie aus dem SAP-System des Universitätsklinikums Jena gewonnen.

Die Dauer zwischen der Diagnosestellung bzw. der ersten SZT bis zur Durchführung der ersten GK-MRT betrug im Mittel 15,4 \pm 39,0 Monate ( 0 - 223,7 Monate; Median $=0,1$ Monate). Bei den Patienten, bei denen keine ON in der GK-MRT detektiert werden konnten, wurden im Mittel 3,6 \pm 3,7 GK-MRT-Aufnahmen (1 - 20; Median = 2) über einen Zeitraum von 16,5 \pm 24,0 Monaten (0 - 82,5 Monate; Median = 3,0 Monate) pro Patient durchgeführt. 40,3 \% der Kinder und Jugendlichen ohne ON erhielten nur einmalig eine Bildgebung. Bei den Patienten mit Entwicklung von ON wurden über einen Zeitraum von 29,4 \pm 23,9 Monaten $(0-82,6$ Monate; Median $=$ 19,8 Monate) im Mittel 6,1 \pm 3,9 GK-MRT-Aufnahmen (1 - 18; Median = 5) pro Patient durchgeführt. Für 11,6 \% der Kinder und Jugendlichen mit ON war nur eine GK-MRT zu finden. Diese Patienten verstarben im Verlauf oder wurden in externen Kliniken weiter betreut, sodass hier keine Verlaufskontrollen vorlagen.

\subsubsection{Patienten ohne Stammzelltransplantation}

Bei 36 Jungen $(57,1 \%)$ und 27 Mädchen (42,9 \%) erfolgte keine SZT. Das Alter bei Diagnosestellung der Grunderkrankung lag zwischen 0,1 und 19,4 Jahren, im Mittel bei 11,1 $\pm 5,6$ Jahre. Für die männlichen Patienten betrug das mittlere Alter 11,0 \pm 5,8 Jahre und für die weiblichen Patienten 11,2 \pm 5,4 Jahre.

Unter den Patienten befanden sich Kinder und Jugendliche, die aufgrund ihrer Grunderkrankungen eine Chemotherapie erhielten, die operativ versorgt wurden oder bei denen der Krankheitsverlauf lediglich beobachtet wurde. Ebenfalls wurden in diese Gruppe auch Patienten mit einer SZT im Verlauf eingeschlossen, bei denen 
das letzte vorhandene GK-MRT noch vor der SZT lag oder die ON bereits in einer GK-MRT vor der SZT auftraten.

Folgende Grunderkrankungen wiesen die 63 Kinder und Jugendlichen ohne SZT auf:

- Morbus Hodgkin

$(\mathrm{n}=20)$

- akute lymphatische Leukämie $\quad(n=13)$

- Neuroblastom

$$
(n=6)
$$

- Non-Hodgkin-Lymphom

$$
(n=5)
$$

- Langerhans-Zell-Histiozytose

- Osteosarkom$$
(n=5)
$$$$
(\mathrm{n}=3)
$$

- Ewing-Sarkom

$(n=2)$

- Rhabdomyosarkom

- akute myeloische Leukämie, Synovialsarkom, myelodysplastisches Syndrom, chronische Granulomatose, hämophagozytische Lymphohistiozytose, PurtiloSyndrom, atypischer teratoider Rhabdoidtumor (jeweils $n=1$ ).

\subsubsection{Patienten mit Stammzelltransplantation}

Unter den insgesamt 42 Patienten mit SZT befanden sich 26 Jungen (61,9\%) und 16 Mädchen (38,1 \%). Das Alter bei der ersten SZT variierte von 0,6 bis 20,0 Jahre mit einem Mittelwert von 10,7 \pm 6,5 Jahren. Das mittlere Alter bei den männlichen Kindern und Jugendlichen betrug bei der ersten SZT 11,1 \pm 6,4 Jahre und bei den weiblichen Patienten 10,1 \pm 6,7 Jahre.

Bei 30 Patienten erfolgte eine Transplantation und bei 12 Patienten zwei SZT. Dabei wurden zusätzliche SZT aufgrund von Abstoßung der initialen Transplantation zu insgesamt einer SZT zusammengefasst. Das betraf drei Patienten in der Gruppe mit einer SZT und zwei Patienten in der Gruppe mit zwei Transplantationen. Dazwischen lagen jeweils ein bis drei Monate, bei einem Patienten jedoch sieben Monate mit überbrückender Chemotherapie bis zum erneuten Finden eines Spenders.

Die Grunderkrankungen der 42 Patienten mit SZT waren folgende:

- akute lymphatische Leukämie $\quad(n=9)$

- akute myeloische Leukämie $\quad(n=7)$

- Neuroblastom $\quad(n=5)$

- Morbus Hodgkin $\quad(n=3)$

- Ewing-Sarkom $\quad(n=3)$

- myelodysplastisches Syndrom $(n=3)$

- Rhabdomyosarkom $\quad(n=2)$ 
- hämophagozytische Lymphohistiozytose, juvenile myelomonozytäre Leukämie, Astroblastom, biphänotypische Leukämie, kombinierte akute myeloische und lymphatische Leukämie, Chediak-Higashi-Syndrom, Weichteilsarkom, myeloproliferatives Syndrom, Nephroblastom, schwere aplastische Anämie (jeweils $n=1$ ).

Von den 42 Patienten erhielten 30 Patienten eine oder mehrere allogene SZT und 12 Patienten eine oder mehrere autologe SZT. Unter den 12 Kindern und Jugendlichen mit autologer SZT befanden sich 3 Patienten, bei denen die erste SZT autolog erfolgte und die nachfolgenden SZT allogen.

\subsection{Bildgebung mittels Ganzkörper-Magnetresonanztomographie}

\subsubsection{Definition Osteonekrosen}

Als ON wurden girlandenförmige Läsionen im Knochen definiert (Abbildung 2). In der T1-nativen Sequenz wurden Läsionen als ON gewertet, die isointens zum umgebenden Knochenmark waren und einen Rand von geringer Signalintensität aufwiesen. In der T2w Sequenz wurden hypointense Läsionen mit einem hyperintensen Rand, der von einem Rahmen geringer Signalintensität umgeben war als ON gewertet. In der T1-Sequenz mit Kontrastmittel und Fettunterdrückung wurden Läsionen als ON definiert, bei denen der Rand einer isointensen Läsion hyperintens war. Fleckige hypointense Läsionen in der T1-nativen Sequenz, die in der T2-nativen Aufnahme und in der T1-Sequenz mit Kontrastmittel und Fettunterdrückung hyperintens erschienen, wurden vor allem im Verlauf zu noch vorhandenen ON erklärt. Ein Fehlen der girlandenförmigen Ausbreitung schloss somit eine ON nicht aus (Zurlo 1999, Beer et al. 2008). 

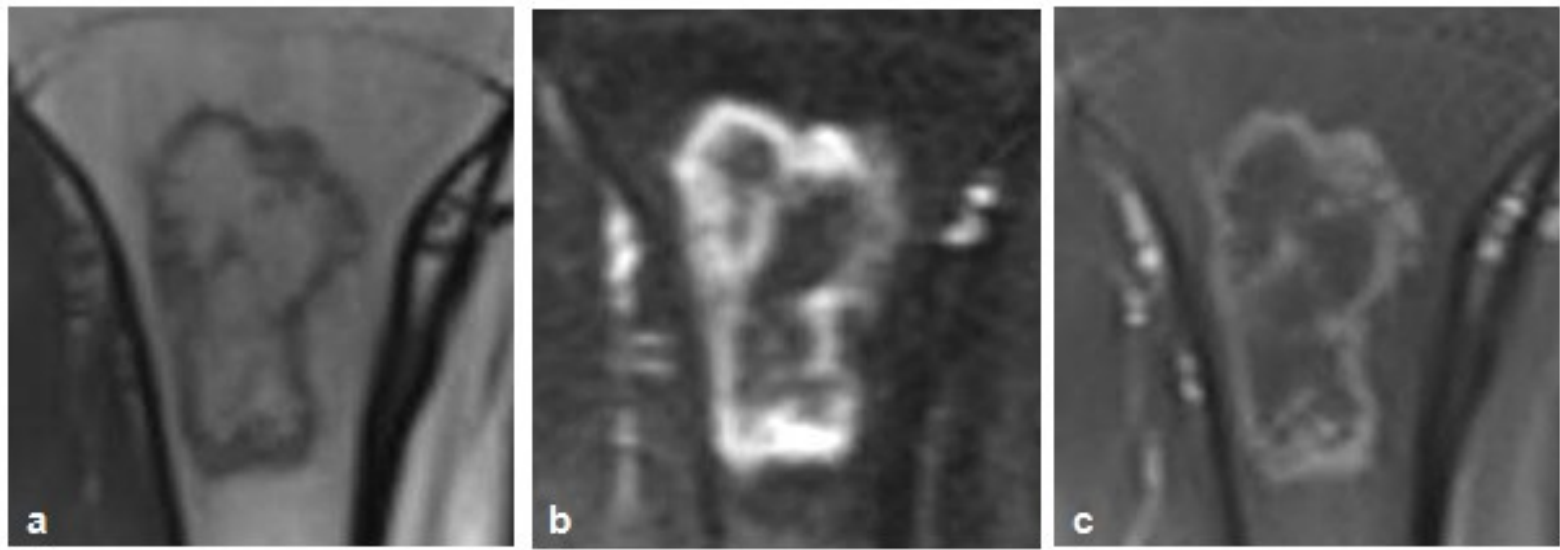

Abbildung 2: Osteonekrose in der rechten proximalen Tibiametaphyse bei einem 17 jährigen Jungen mit Morbus Hodgkin - Darstellung in der T1-SPACE- (a), T2-HASTEIR- (b) und T1-SPACE-SPAIR-Sequenz mit Kontrastmittel (c)

\title{
3.2.2 Durchführung der GK-MRT
}

Die Indikationen für die Durchführung der ersten GK-MRT-Untersuchung unter den 105 Patienten sind in Abbildung 3 ersichtlich.

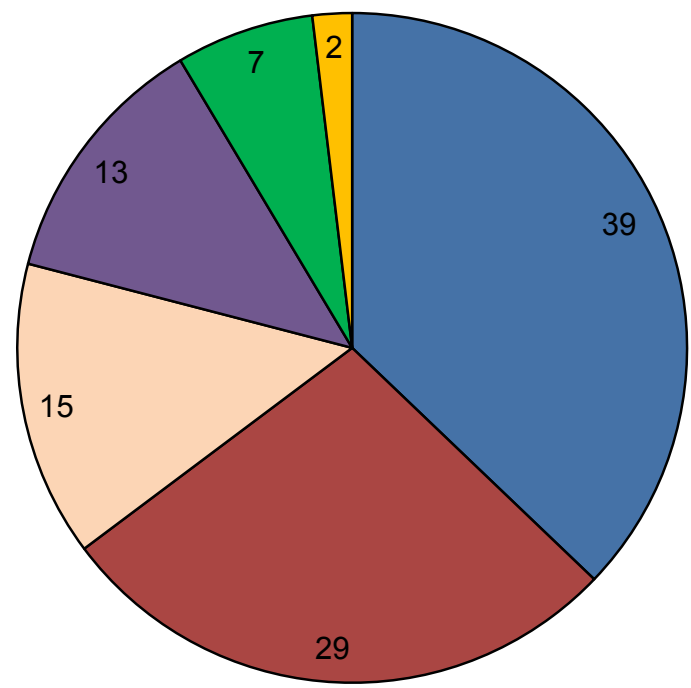

\author{
$\square$ Diagnosestellung der \\ Grunderkrankung \\ $\square$ Routine-Verlaufskontrolle im \\ Rahmen der Grunderkrankung \\ $\square$ Schmerzsymptomatik \\ $\square$ Diagnostik der Grunderkrankung \\ oder Diagnostik vor SZT \\ (Erkrankung bekannt; Staging) \\ $\square$ Diagnostik bei bestätigtem Rezidiv \\ oder Rezidivverdacht bei z.B. \\ suspekten Lymphknoten \\ $\square$ Verlauf bekannter ON (bereits \\ früher in MRT gesehen)
}

\section{Abbildung 3: Indikationen für die Durchführung der ersten GK-MRT}

Technische Daten der GK-MRT sowie die Qualitätsbeurteilung der Sequenzen hinsichtlich der Darstellung von ON wurden bei den Kindern und Jugendlichen erfasst, bei denen ON in der GK-MRT detektiert werden konnten. Die Bildgebungen dieser Kinder und Jugendlichen erfolgten an zwei verschiedenen MRT-Geräten mit einer magnetischen Flussdichte von 1,5 T (Siemens Avanto und Siemens 
Symphony, Erlangen, Germany). Drei der Patienten wurden in Narkose am SiemensSymphony-Gerät untersucht, 40 Kinder und Jugendliche am Siemens-Avanto-Gerät. Bei einem der Patienten war die Untersuchung in Narkose nur beim ersten GK-MRT mit Nachweis von ON nötig, im weiteren Verlauf war dies nicht mehr erforderlich. In allen Fällen wurden Ganzkörper- oder Phased-Array-Spulen sowie die automatische Tischverschiebung verwendet. Die Patienten befanden sich in Rückenlage auf dem Untersuchungstisch des Magnetresonanztomographen mit seitlich an den Körper angelegten Armen, wobei die Hände auf den Oberschenkeln bzw. im Schoß abgelegt wurden.

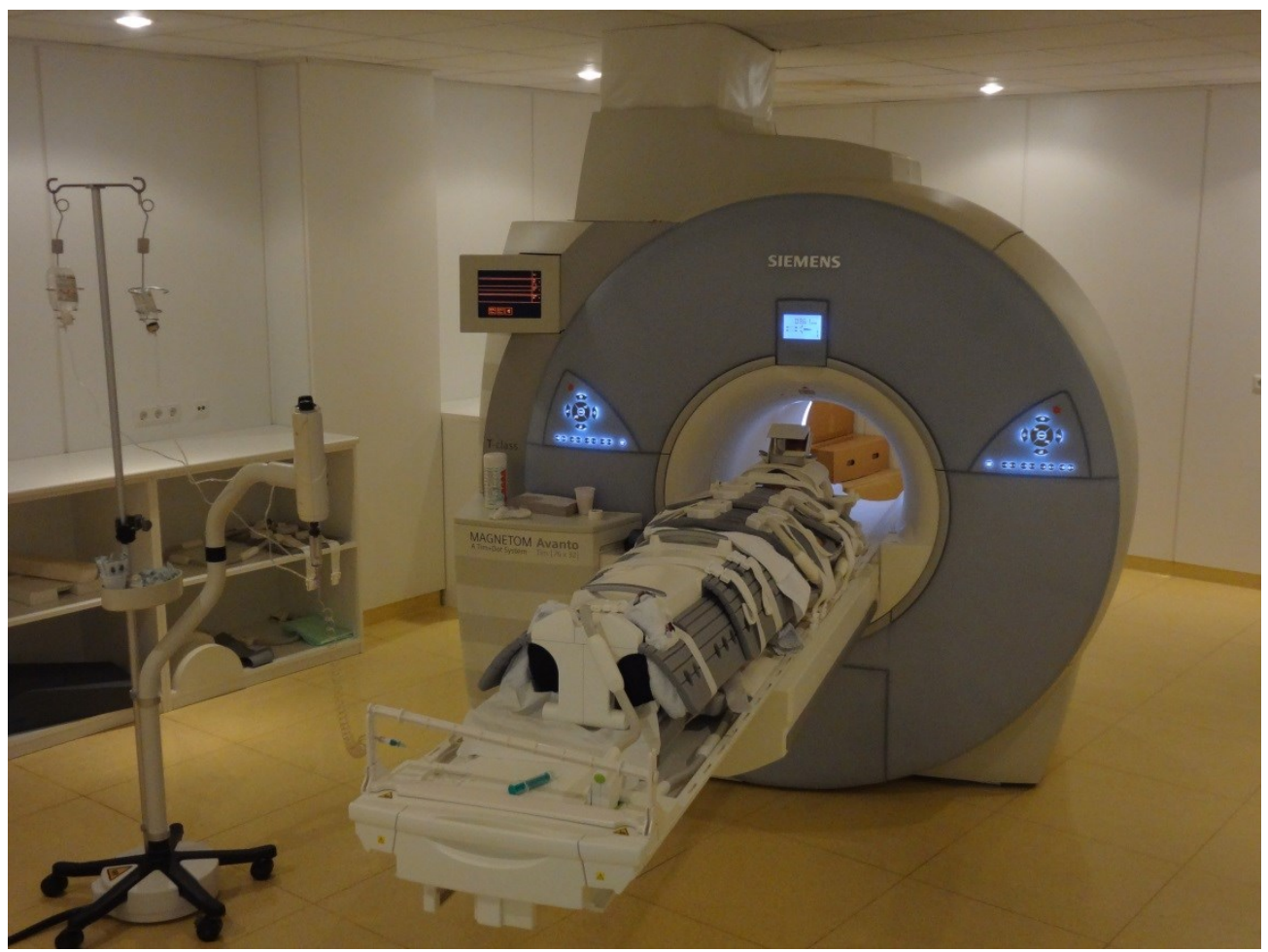

Abbildung 4: Lagerung des Patienten und Positionierung der Spulen für eine GK-MRT im MRT-Gerät Siemens Avanto (Universitätsklinikum Jena)

Der Körper wurde je nach Körpergröße in eine bestimmte Anzahl an Abschnitten aufgeteilt. Für den größten Teil der Patienten waren es fünf Abschnitte:

1. Kopf bis Schulter

2. Schulter mit Armen sowie Thorax und Abdomen

3. Becken bis proximale Oberschenkel (Hüftregion) und Unterarme mit Händen

4. distale Oberschenkel bis proximale Unterschenkel (Knieregion)

5. distale Unterschenkel bis Fuß. 
Weitere Aufteilungen erfolgten bei entsprechend kleineren Kindern in vier Abschnitte sowie bei Kleinkindern und Säuglingen in drei Abschnitte.

Die Bilder wurden retrospektiv im Konsens von einem Kinderradiologen mit 10 Jahren Erfahrung in der GK-MRT und nach entsprechender mehrwöchiger Einweisung im Rahmen einer Famulatur von der Doktorandin begutachtet und ausgewertet.

\subsubsection{Qualitätsbewertung zur Darstellung von Osteonekrosen}

Um zu beurteilen, wie gut sich die ON in den einzelnen Sequenzen qualitativ darstellen lassen, wurde das jeweils erste GK-MRT mit Nachweis von ON im Patienten, welches am Universitätsklinikum Jena durchgeführt wurde, betrachtet. Dabei wurden vier Sequenzen verglichen:

1. T1-SPACE (= Sampling perfection with application optimized contrasts using different flip angle evolutions): Schichtdicke (SD) $2 \mathrm{~mm}$, Gap $0 \mathrm{~mm}$, Repetitionszeit (TR) $430 \mathrm{~ms}$, Echozeit (TE) $11 \mathrm{~ms}$, Flipwinkel 120, Matrix 448 $x 448$

2. T2-HASTE (= Half Fourier Acquired Single Shot Turbo Spin Echo): SD $5 \mathrm{~mm}$, Gap $1 \mathrm{~mm}$, TR $1100 \mathrm{~ms}$, TE $118 \mathrm{~ms}$, Flipwinkel 150, Matrix 448 x 448

3. T2-HASTE-IR (IR = Inversion Recovery): SD $5 \mathrm{~mm}$, Gap $1 \mathrm{~mm}$, TR $1100 \mathrm{~ms}$, TE $61 \mathrm{~ms}$, Inversionszeit (TI) 180 ms, Flipwinkel 150, Matrix 384 x 384

4. T1-SPACE-SPAIR mit Kontrastmittel (SPAIR = Spectral presaturation with adiabatic Inversion Recovery): SD 2 mm, Gap 0 mm, TR 430 ms, TE 11 ms, Flipwinkel $120^{\circ}$, Matrix $448 \times 448$. Als Kontrastmittel wurden makrozyklische Gadolinium-DTPA-Präparate (Gadovist ${ }^{\circledR}$, Bayer oder Dotarem ${ }^{\circledR}$, Guerbet) in einer Dosierung von 0,1 mmol/kg Körpergewicht intravenös verwendet.

Die Qualitätsbewertung zum Nachweis von ON in den einzelnen MRT-Sequenzen erfolgte angelehnt an das System von Mirowitz et al. (Mirowitz et al. 1994). Die berücksichtigten Kriterien waren dabei Sichtbarkeit, Abgrenzbarkeit, diagnostische Sicherheit, Ausdehnung, Bildqualität und Bewegungsartefakte. Angelehnt an eine Likert-Skala mit den Kategorien 1 = schlecht, $2=$ moderat, $3=$ gut und $4=$ exzellent wurde für jedes Item, was in dieser Studie jeder einzelnen ON entspricht, in den jeweils vorhandenen Sequenzen ein Wert pro Kriterium bestimmt. Auf Item- bzw. Nekrosenebene wurde der Mittelwert als Qualitätsscore für jede Sequenz errechnet und mit den Werten der anderen Sequenzen verglichen. Analog dazu wurde die diagnostische Sicherheit als einzelnes Kriterium für jede $\mathrm{ON}$ zwischen den 
Sequenzen analysiert. Nicht bei jedem Patienten waren Aufnahmen aus allen vier Sequenzen vorhanden, demnach konnten entsprechend bei diesen Patienten nicht alle Vergleiche durchgeführt werden. Im Ergebnisteil wird darauf verwiesen.

\subsubsection{Lokalisation von Osteonekrosen}

Für die Auswertung der Lokalisation der $\mathrm{ON}$ wurden alle möglichen aufgetretenen Läsionen unabhängig vom Zeitpunkt der ersten Manifestation in der GK-MRT erfasst. Jede ON wurde anhand des Knochens, in dem sie verifiziert wurde, der Körperseite und bei den langen Röhrenknochen (Humerus, Radius, Ulna, Femur, Tibia, Fibula) anhand der Lage im Knochen (Abbildung 5) beschrieben. Daraus erfolgte nicht nur die Analyse, wie viele Lokalisationen pro Patient auftraten, sondern auch hinsichtlich der Häufigkeit des Auftretens in der oberen oder unteren Extremität, auf der rechten oder linken Körperseite und in der Epi-, Meta- oder Diaphyse. Nekrosen, die über mehrere Regionen im Knochen reichten, wurden entsprechend danach benannt.

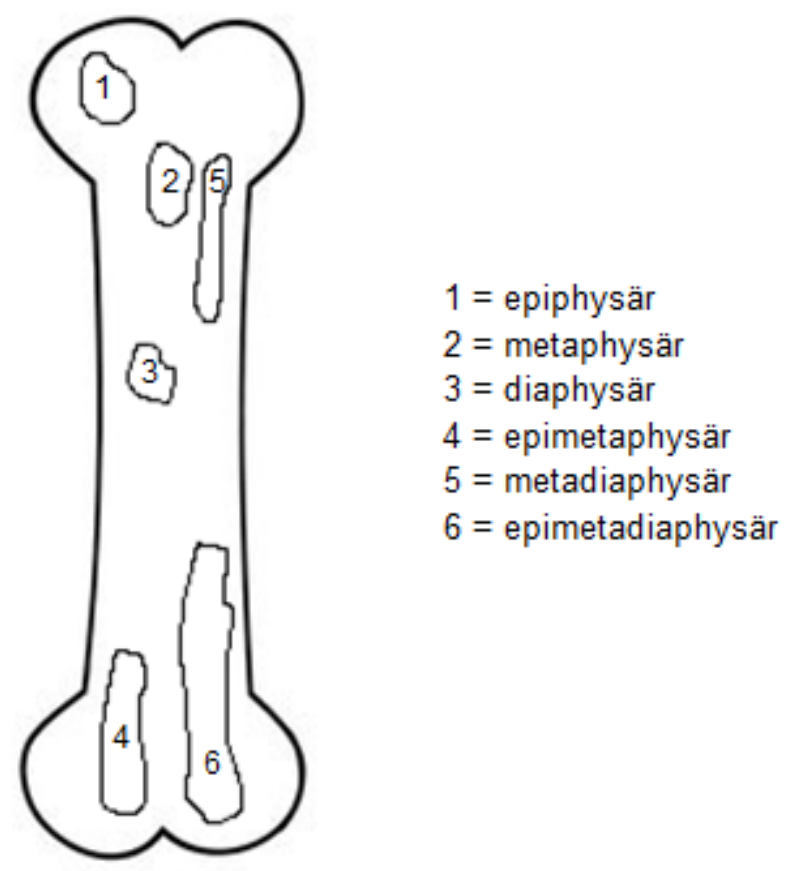

Abbildung 5: Definition der Lage der Osteonekrosen in den langen Röhrenknochen

Für alle Läsionen mit Anteil an der Epiphyse wurde die Gelenkflächenbeteiligung bestimmt. Verschiedene Lokalisationen im Becken wurden als „Becken“, in den Karpalknochen als „Hand“ und in den Tarsalknochen als „Fuß“ bezeichnet.

Um die Häufigkeit des Auftretens der $\mathrm{ON}$ in bestimmten Knochen und zu einem Gelenk angrenzenden Knochen zu beurteilen, wurden die Lokalisationen wie folgt zusammengefasst: Alle $\mathrm{ON}$ in der Diaphyse der langen Röhrenknochen wurden 
entsprechend des Knochens bezeichnet, Läsionen in der Meta- und Epiphyse nach dem angrenzenden Gelenk. Die Nekrosen, welche von der Dia- oder Metaphyse bis in die Epiphyse reichten, wurden zum nächstgelegenen Gelenk dazugerechnet.

Folgende weitere Definitionen erfolgten:

- $\quad \mathrm{ON}$ im Acromion $\triangleq$ Schulter

- $\quad \mathrm{ON}$ in den Karpalknochen $\triangleq$ Hand

- $\mathrm{ON}$ im Acetabulum $\triangleq$ Hüfte

- $\quad$ ON im Becken (außer Acetabulum) ^ Becken

- $\quad$ ON in der Patella $\triangleq$ Patella

- $\quad$ ON im Talus $\triangleq$ oberes Sprunggelenk (OSG)

- $\quad$ ON in den Tarsalknochen (außer Talus) $\triangleq$ Fuß.

Mehrere Lokalisationen in einem Knochen oder den an ein Gelenk angrenzenden Knochenregionen wurden als eine ON zusammengefasst. Jede Körperseite wurde getrennt betrachtet.

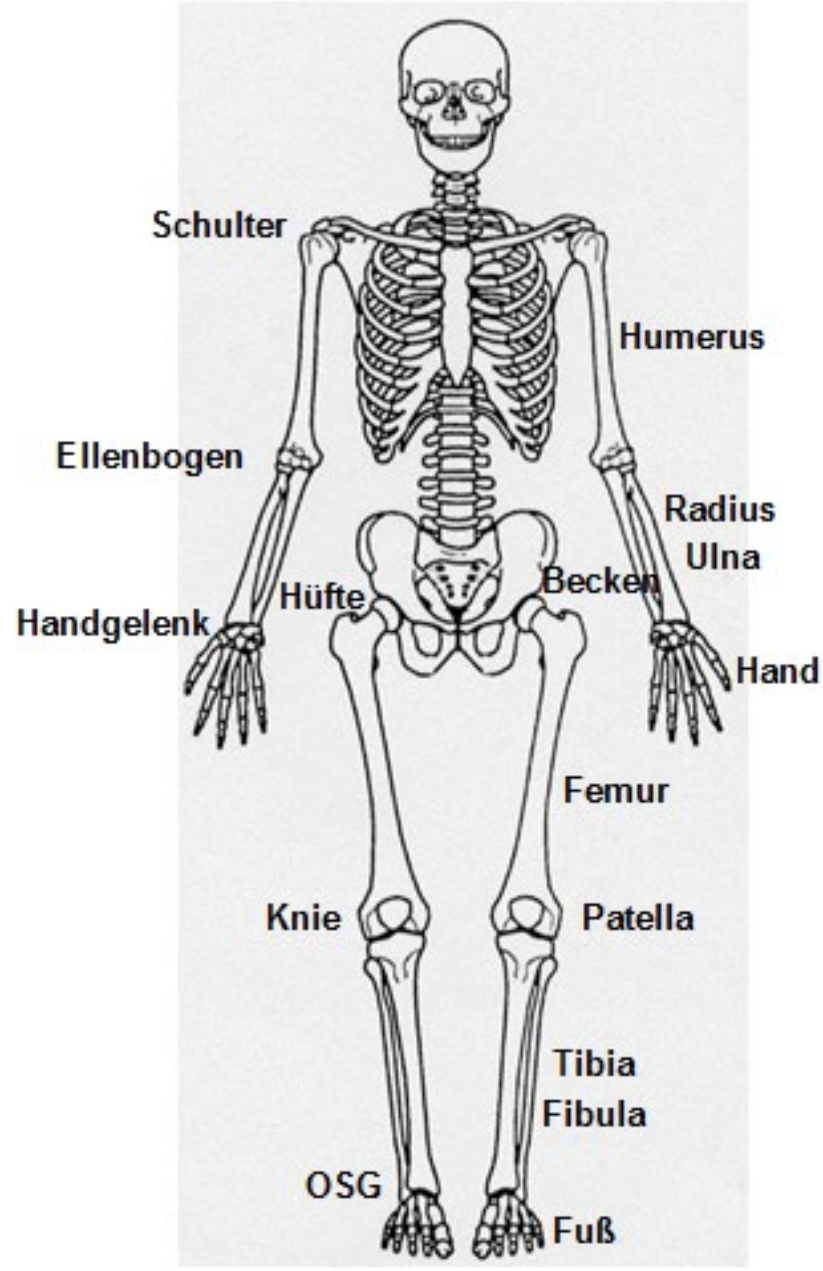

Abbildung 6: Aufteilung der Lokalisationen der Osteonekrosen nach Knochen (rechts) und gelenknahen Knochenregionen (links) (Giesecke 2002) 


\subsubsection{Patientenspezifische Einflussfaktoren auf den positiven Nachweis von Osteonekrosen in der GK-MRT}

Die Häufigkeit des positiven Nachweises von ON in der GK-MRT sowie die Dauer zwischen der Diagnose der Grunderkrankung (Patienten ohne SZT) bzw. der ersten SZT (Patienten mit SZT) und dem ersten ON-Nachweis in der GK-MRT wurden für die Gruppen mit und ohne SZT bestimmt. Ebenfalls wurde erfasst, ob die Patienten zum Zeitpunkt der ersten GK-MRT mit ON-Auftreten eine Schmerzsymptomatik aufwiesen. Das Symptom Schmerz wurde gewertet, sobald in der Akte ein Knochenschmerz unabhängig von der Lokalisation dokumentiert wurde.

In mehreren Studien so z.B. von Girard et al. (Girard et al. 2013) und Kawedia et al. (Kawedia et al. 2011) konnte ein signifikant erhöhtes Risiko für das Auftreten von ON bei Kindern > 10 Jahren gefunden werden. Angelehnt daran wurde in der vorliegenden Arbeit in den beiden Patientengruppen der Einfluss des Alters bei Diagnosestellung der Grunderkrankung (Patienten ohne SZT) oder bei der ersten SZT (Patienten mit SZT) aufgeteilt in die Altersgruppen kleiner gleich und größer als zehn Jahre analysiert. Als weitere Variable wurde der Einfluss des Geschlechts der Patienten auf den positiven Nachweis von ON in der GK-MRT untersucht.

Für die Gruppe der Kinder und Jugendlichen ohne SZT wurden die Häufigkeiten der ON-Manifestation in der GK-MRT nach den jeweiligen Grunderkrankungen ermittelt. Ebenfalls wurden für diese Patientengruppe die verabreichte kumulative Steroiddosis erfasst. Die verabreichten Steroide waren Prednisolon, Dexamethason oder eine Kombination von beiden. Der Einfluss der Art des gegebenen Steroids sowie die Höhe der verabreichten Dosis auf das Auftreten von ON in der GK-MRT wurden unabhängig von der Grunderkrankung analysiert. Neben der kumulativen Steroiddosis wurde auch der Einfluss der Dosis der einzelnen Steroidarten analysiert. Zur Berechnung der insgesamt verabreichten Dosis wurde die Dexamethasondosis mit dem Faktor 6,67 in ein Prednisolonäquivalent umgewandelt (Inaba und Pui 2010). Die Vergleiche erfolgten jeweils zwischen drei Gruppen von Patienten abhängig von der Höhe der verabreichten Steroiddosis. Die Aufteilungen in die Patientengruppen sind in Tabelle 2 dargestellt. 
Tabelle 2: Aufteilung der Patienten in Abhängigkeit von der Höhe der verabreichten Steroiddosis

\begin{tabular}{|c|c|c|c|}
\hline Patientengruppe & $\begin{array}{c}\text { Kumulative } \\
\text { Steroiddosis } \\
\text { in } \mathrm{g} / \mathrm{m}^{2}\end{array}$ & $\begin{array}{l}\text { Prednisolon- } \\
\text { dosis } \\
\text { in } \mathrm{g} / \mathrm{m}^{2}\end{array}$ & $\begin{array}{c}\text { Dexamethason- } \\
\text { dosis } \\
\text { in } \mathrm{g} / \mathrm{m}^{2}\end{array}$ \\
\hline keine Steroide & 0 & 0 & 0 \\
\hline niedrigere Dosis & $\leq 2,8$ & $\leq 2,0$ & $\leq 0,3$ \\
\hline höhere Dosis & $>2,8$ & $>2,0$ & $>0,3$ \\
\hline
\end{tabular}

\subsubsection{Zeitlicher Verlauf der Osteonekrosen}

Bei den Patienten mit positivem Nachweis von ON in der GK-MRT wurden über einen Zeitraum von 24,4 \pm 24,2 Monaten ( 0 - 80,2 Monate; Median = 15,2 Monate) die Läsionen im Verlauf beobachtet. Bei $79,1 \%$ der Patienten mit ON wurden nach dem erstmaligen Nachweis von ON in der GK-MRT weitere GK-MRT-Aufnahmen durchgeführt, 20,9 \% erhielten keine weiteren Untersuchungen am Universitätsklinikum Jena. Die Verlaufsbildgebung wurde ab dem ersten GK-MRT mit ON-Nachweis in möglichst halbjährlichen Intervallen ausgewertet. Wenn mehrere Aufnahmen in einem halben Jahr angefertigt wurden, wurde demzufolge nur eine davon berücksichtigt. Mindestens eine GK-MRT-Verlaufsbildgebung im entsprechenden zeitlichen Abstand war bei 22 Kindern und Jugendlichen ohne SZT und bei 12 Patienten mit SZT vorhanden.

Für die Beurteilung des Verlaufs der $\mathrm{ON}$ wurden für die weitere Analyse anschließend nur die 20 Patienten mit Morbus Hodgkin als Grunderkrankung und ohne SZT eingeschlossen. Bei 16 dieser 20 Hodgkin-Patienten waren ON in der GKMRT detektiert worden. Von diesen 16 Patienten musste eine Patientin ausgeschlossen werden, da sie sich im Verlauf einer SZT unterziehen musste. Bei den restlichen 15 Patienten wurde eine mögliche Therapie hinsichtlich der ON berücksichtigt. Ab dem ersten Zeitpunkt einer ON-Behandlung wurden die Patienten als „unter Therapie“ definiert. Dabei war diese Aussage unabhängig davon, welche Therapie stattfand, wie lange diese durchgeführt wurde und ob noch mehrere Behandlungen, womöglich auch unterschiedliche, im Verlauf stattfanden.

Die ON wurden für die Verlaufsbeurteilung auf der koronaren GK-MRT-Aufnahme in zwei Richtungen (kraniokaudal, mediolateral) vermessen ( $=$ a und b). Nach entsprechend axialer Rekonstruktion konnte auch die sagittale Ausbreitung 
(anterioposterior $=\mathrm{c}$ ) der Nekrose in $\mathrm{mm}$ erfasst werden. Aus diesen Werten wurde mit Hilfe der Ellipsoidformel das Volumen der Läsion in $\mathrm{cm}^{3}=\mathrm{ml}$ berechnet:

$$
V=\frac{4}{3} \pi \mathrm{abc} / 1000 \text {. }
$$

In halbjährlichen Abständen zum initialen GK-MRT mit ON-Nachweis wurde die Summe der Volumina aller aufgetretenen $\mathrm{ON}$ in einem Patienten graphisch dargestellt. Dabei konnte es vorkommen, dass die Anzahl der betrachteten ON zu den jeweiligen Zeitpunkten variierte. Nicht bei jedem Patienten war für jeden halbjährlichen Abstand ein GK-MRT vorhanden. Die fehlenden Werte zwischen zwei vorhandenen Summen der Volumina wurden graphisch interpoliert.

\subsection{Statistische Auswertung}

Die Auswertung des Qualitätsscore sowie der diagnostischen Sicherheit der einzelnen MRT-Sequenzen auf Nekrosenebene erfolgte mittels des nicht parametrischen Vorzeichentests.

Die Häufigkeit des Auftretens der ON in der GK-MRT sowie die verschiedenen Lokalisationen wurden deskriptiv beurteilt.

Um den Einfluss des Alters bei Diagnose der Grunderkrankung (Patienten ohne SZT) bzw. bei der ersten SZT (Patienten mit SZT) und des Geschlechts auf den positiven Nachweis von ON in der GK-MRT zu beurteilen, wurden die Ergebnisse mit Hilfe der Kaplan-Meier-Methode und des Log-Rank-Tests sowie der Cox-Regression analysiert. Diese Analyseverfahren wurden ebenfalls genutzt, um herauszufinden, ob das Auftreten der ON in der GK-MRT durch die Art des verabreichten Steroids sowie die Höhe der kumulativen Steroiddosis bei den Patienten ohne SZT beeinflusst wurde. Ein statistischer Vergleich zwischen Ergebnissen der Patienten ohne und mit SZT konnte nicht erfolgen, da als Ausgangspunkte einmal das Datum der Diagnosestellung der Grunderkrankung und einmal die erste SZT gewählt wurden. Im Ergebnisteil wurde deshalb an diesen Stellen kein Signifikanzwert angegeben.

Der Verlauf der Summe der Volumina aller $\mathrm{ON}$ in einem Patienten wurde in Abhängigkeit von der Zeit jeweils graphisch dargestellt.

Die statistische Analyse mit einem Signifikanzniveau von $\alpha=0,05$ erfolgte mit IBM SPSS Statistics Version 23.0. 


\section{Ergebnisse}

\subsection{Qualitätsbewertung der einzelnen Sequenzen im Nachweis von Osteonekrosen}

In die Qualitätsbewertung sowie die Beurteilung der diagnostischen Sicherheit für die vier vorhandenen Sequenzen wurden 43 Kinder und Jugendliche mit insgesamt 392 ON eingeschlossen.

Die Dauer der jeweiligen Sequenzen betrug für die Ganzkörperdarstellung im Mittel 9,1 $\min (4-12 \mathrm{~min}$; Median = $10 \mathrm{~min}$ ) für die native T1-SPACE-Sequenz, 7,6 min (5 - 10 min; Median = $7 \mathrm{~min}$ ) für die T2-HASTE-Sequenz, 7,1 min (4 - 10 min; Median = $7 \mathrm{~min}$ ) für die T2-HASTE-IR-Sequenz und 9,9 $\mathrm{min}$ (6 - $19 \mathrm{~min}$; Median = $10 \mathrm{~min}$ ) für die T1-SPACE-SPAIR-Sequenz mit Kontrastmittel. Die Sequenzen dauerten in der Regel bei fünf Körperabschnitten so lange, wie es der Median anzeigt. Die starke Abweichung von $4 \mathrm{~min}$ in der T1-nativen Sequenz lag darin begründet, dass bei einem Patienten in dieser Sequenz nur zwei von drei Körperabschnitten abgebildet wurden. Bei dem gleichen Patienten war auch die starke Abweichung von $19 \mathrm{~min}$ in der T1-Sequenz mit Kontrastmittel zu finden. Zwischen den ersten beiden und dem dritten Körperabschnitt bei dieser Sequenz wurde noch eine andere Sequenz zur Schädeldarstellung durchgeführt. Die gesamte GK-MRT-Untersuchung pro Patient mit teils einer aber auch mehr als den beschriebenen Sequenzen dauerte im Mittel $58,1 \mathrm{~min} \pm 16,1 \mathrm{~min}(8-90 \mathrm{~min}$; Median $=57 \mathrm{~min})$, wobei nur die reine Planungsund Messzeit aber nicht die Belegungszeit des Scannerraums betrachtet wurde. Die Dauer der Platzierung von Patient und Spulenelementen war stark von der Compliance der Kinder abhängig und hatte bis zu 20 Minuten in Anspruch genommen.

Je nach Körpergröße wurde der Körper zur GK-MRT-Untersuchung in eine bestimmte Anzahl an Abschnitten geteilt. Bei 30 Patienten mit einer medianen Körpergröße von $170 \mathrm{~cm}(120-185 \mathrm{~cm})$ waren es 5 Stapel. Bei 11 Patienten wurde in 4 Abschnitte geteilt. Die mediane Körpergröße betrug dabei $150 \mathrm{~cm}(113-171$ $\mathrm{cm})$. Bei den zwei kleinsten Kindern mit einer medianen Größe von 104 cm (98 - 110 $\mathrm{cm}$ ) wurden nur 3 Stapel benötigt. 
Die Anzahl der betrachteten ON in der jeweiligen Sequenz waren folgende:

- T1-nativ:

$\mathrm{n}=364$

- T2-HASTE:

$n=139$

- T2-HASTE-IR: $\quad \mathrm{n}=210$

- $\mathrm{T} 1+\mathrm{KM}:$

$n=353$

In den Tabellen 3 und 4 sind zunächst die Auswertungen zum Qualitätsscore dargestellt. Der Qualitätsscore bildet sich wie in 3.2.3 beschrieben aus dem Mittelwert der sechs betrachteten Kriterien.

Tabelle 3: Daten zum Qualitätsscore (QS) in den einzelnen Sequenzen (SD = Standardabweichung; KM = Kontrastmittel)

\begin{tabular}{|c|c|c|}
\hline Sequenz & QS Mittelwert \pm SD & Häufigkeit QS = 4,0 \\
\hline T1-nativ & $3,83 \pm 0,33$ & $66,2 \%$ \\
\hline T2-HASTE & $3,31 \pm 0,71$ & $26,6 \%$ \\
\hline T2-HASTE-IR & $3,59 \pm 0,51$ & $38,1 \%$ \\
\hline T1 + KM & $3,82 \pm 0,38$ & $65,7 \%$ \\
\hline
\end{tabular}

Der Qualitätsscore von 4,0 wurde in allen vier Sequenzen für die insgesamt 392 analysierten Läsionen am häufigsten erreicht. Ebenfalls ist an den Mittelwerten ersichtlich, dass die Darstellung von ON in der GK-MRT in allen Wichtungen gut bis sehr gut ist.

In Tabelle 4 sind die Ergebnisse des Vorzeichentests zusammengefasst. Dabei wurde der Qualitätsscore der jeweiligen Sequenzen gegenübergestellt. Die Sequenzen T2-HASTE und T2-HASTE-IR wurden nicht miteinander verglichen, da nur eine der beiden Sequenzen pro Patient vorlag. 
Tabelle 4: Vergleich des Qualitätsscore (QS) zwischen den einzelnen Sequenzen (KM = Kontrastmittel)

\begin{tabular}{|c|c|c|c|}
\hline Sequenzvergleich & QS-Wert & Anzahl der Werte & Signifikanz \\
\hline \multirow{4}{*}{$\begin{array}{l}\text { T1 vs. } \\
\text { T2-HASTE }\end{array}$} & Gesamt & $n=139$ & \multirow[t]{4}{*}{$p<0,001$} \\
\hline & $\mathrm{T} 1>\mathrm{T} 2$ & $\mathrm{n}=84$ & \\
\hline & $\mathrm{T} 1<\mathrm{T} 2$ & $\mathrm{n}=9$ & \\
\hline & $\mathrm{T} 1=\mathrm{T} 2$ & $\mathrm{n}=46$ & \\
\hline \multirow{4}{*}{$\begin{array}{c}\text { T1 vs. } \\
\text { T2-HASTE-IR }\end{array}$} & Gesamt & $\mathrm{n}=182$ & \multirow[t]{4}{*}{$p<0,001$} \\
\hline & $\mathrm{T} 1>\mathrm{T} 2-\mathrm{IR}$ & $\mathrm{n}=82$ & \\
\hline & $\mathrm{T} 1<\mathrm{T} 2-\mathrm{IR}$ & $\mathrm{n}=9$ & \\
\hline & $\mathrm{T} 1=\mathrm{T} 2-\mathrm{IR}$ & $\mathrm{n}=91$ & \\
\hline \multirow{4}{*}{$\begin{array}{c}\text { T1 vs. } \\
\text { T1 + KM }\end{array}$} & Gesamt & $n=343$ & \multirow[t]{4}{*}{$p=0,853$} \\
\hline & $\mathrm{T} 1>\mathrm{T} 1+\mathrm{KM}$ & $n=60$ & \\
\hline & $\mathrm{T} 1<\mathrm{T} 1+\mathrm{KM}$ & $\mathrm{n}=57$ & \\
\hline & $\mathrm{T} 1=\mathrm{T} 1+\mathrm{KM}$ & $n=226$ & \\
\hline \multirow{4}{*}{$\begin{array}{c}\text { T2- HASTE vs. } \\
\text { T1 + KM }\end{array}$} & Gesamt & $n=139$ & \multirow[t]{4}{*}{$p<0,001$} \\
\hline & $\mathrm{T} 2>\mathrm{T} 1+\mathrm{KM}$ & $\mathrm{n}=9$ & \\
\hline & $\mathrm{T} 2<\mathrm{T} 1+\mathrm{KM}$ & $\mathrm{n}=82$ & \\
\hline & $\mathrm{T} 2=\mathrm{T} 1+\mathrm{KM}$ & $\mathrm{n}=48$ & \\
\hline \multirow{4}{*}{$\begin{array}{c}\text { T2-HASTE-IR vS. } \\
\text { T1 + KM }\end{array}$} & Gesamt & $\mathrm{n}=171$ & \multirow[t]{4}{*}{$p<0,001$} \\
\hline & $\mathrm{T} 2-\mathrm{IR}>\mathrm{T} 1+\mathrm{KM}$ & $\mathrm{n}=7$ & \\
\hline & $\mathrm{T} 2-\mathrm{IR}<\mathrm{T} 1+\mathrm{KM}$ & $\mathrm{n}=92$ & \\
\hline & $\mathrm{T} 2-\mathrm{IR}=\mathrm{T} 1+\mathrm{KM}$ & $n=72$ & \\
\hline
\end{tabular}

Die Ergebnisse zur diagnostischen Sicherheit sind ähnlich. Dabei ist die diagnostische Sicherheit ein einzelnes Kriterium, welches die Werte 1 - 4 pro Sequenz und ON annehmen konnte.

Tabelle 5: Daten zur diagnostischen Sicherheit (DS) in den einzelnen Sequenzen (SD = Standardabweichung; KM = Kontrastmittel)

\begin{tabular}{|c|c|c|}
\hline Sequenz & DS Mittelwert $\mathbf{\text { SD }}$ & Häufigkeit DS = 4 \\
\hline T1-nativ & $3,62 \pm 0,70$ & $72,5 \%$ \\
\hline T2-HASTE & $2,74 \pm 1,15$ & $34,5 \%$ \\
\hline T2-HASTE-IR & $3,13 \pm 0,91$ & $44,3 \%$ \\
\hline T1 + KM & $3,61 \pm 0,72$ & $72,5 \%$ \\
\hline
\end{tabular}


Der Wert 4, der einer exzellenten Darstellung einer ON entspricht, wurde in allen vier Sequenzen am häufigsten vergeben.

In Tabelle 6 sind die Ergebnisse zum Vergleich des Werts der diagnostischen Sicherheit zwischen den Sequenzen mit Hilfe des Vorzeichentests zusammengefasst. Wie schon beim Vergleich des Qualitätsscore wurden die Sequenzen T2-HASTE und T2-HASTE-IR auch hier nicht miteinander verglichen.

Tabelle 6: Vergleich der Werte zur diagnostischen Sicherheit (DS) zwischen den Sequenzen (KM = Kontrastmittel)

\begin{tabular}{|c|c|c|c|}
\hline Sequenzvergleich & DS-Wert & Anzahl der Werte & Signifikanz \\
\hline \multirow{4}{*}{$\begin{array}{c}\text { T1 vs. } \\
\text { T2-HASTE }\end{array}$} & Gesamt & $\mathrm{n}=139$ & \multirow[t]{4}{*}{$p<0,001$} \\
\hline & $\mathrm{T} 1>\mathrm{T} 2$ & $\mathrm{n}=71$ & \\
\hline & $\mathrm{T} 1<\mathrm{T} 2$ & $\mathrm{n}=4$ & \\
\hline & $\mathrm{T} 1=\mathrm{T} 2$ & $n=64$ & \\
\hline \multirow{4}{*}{$\begin{array}{c}\text { T1 vs. } \\
\text { T2-HASTE-IR }\end{array}$} & Gesamt & $\mathrm{n}=182$ & \multirow[t]{4}{*}{$p<0,001$} \\
\hline & T1 > T2-IR & $\mathrm{n}=67$ & \\
\hline & T1 > T2-IR & $\mathrm{n}=7$ & \\
\hline & T1 > T2-IR & $\mathrm{n}=108$ & \\
\hline \multirow{4}{*}{$\begin{array}{c}\text { T1 vs. } \\
\text { T1 + KM }\end{array}$} & Gesamt & $n=343$ & \multirow[t]{4}{*}{$p=0,386$} \\
\hline & $\mathrm{T} 1>\mathrm{T} 1+\mathrm{KM}$ & $n=47$ & \\
\hline & $\mathrm{T} 1<\mathrm{T} 1+\mathrm{KM}$ & $\mathrm{n}=38$ & \\
\hline & $\mathrm{T} 1=\mathrm{T} 1+\mathrm{KM}$ & $n=258$ & \\
\hline \multirow{4}{*}{$\begin{array}{c}\text { T2-HASTE vs. } \\
\text { T1 + KM }\end{array}$} & Gesamt & $n=139$ & \multirow[t]{4}{*}{$p<0,001$} \\
\hline & $\mathrm{T} 2>\mathrm{T} 1+\mathrm{KM}$ & $n=6$ & \\
\hline & $\mathrm{T} 2<\mathrm{T} 1+\mathrm{KM}$ & $\mathrm{n}=68$ & \\
\hline & $\mathrm{T} 2=\mathrm{T} 1+\mathrm{KM}$ & $n=65$ & \\
\hline \multirow{4}{*}{$\begin{array}{c}\text { T2-HASTE-IR vs. } \\
\text { T1 + KM }\end{array}$} & Gesamt & $n=171$ & \multirow[t]{4}{*}{$p<0,001$} \\
\hline & $\mathrm{T} 2-\mathrm{IR}>\mathrm{T} 1+\mathrm{KM}$ & $\mathrm{n}=3$ & \\
\hline & $\mathrm{T} 2-\mathrm{IR}<\mathrm{T} 1+\mathrm{KM}$ & $\mathrm{n}=74$ & \\
\hline & $\mathrm{T} 2-\mathrm{IR}=\mathrm{T} 1+\mathrm{KM}$ & $\mathrm{n}=94$ & \\
\hline
\end{tabular}




\subsection{Positiver Nachweis von Osteonekrosen in der GK-MRT und Bezug zu klinischer Symptomatik}

Insgesamt konnte bei 43 der 105 Kinder und Jugendlichen (41,0 \%) im Rahmen der GK-MRT mindestens eine ON im Körper nachgewiesen werden. Bei den 63 Jungen und Mädchen ohne SZT trat bei 26 Patienten (41,3\%) mit einem medianen Intervall von 6,0 Monaten (0 - 80,5 Monate) zwischen Diagnose der Grunderkrankung und dem ersten Nachweis von ON in der GK-MRT mindestens eine ON auf. Bei den 42 Kindern und Jugendlichen mit SZT fand sich bei 17 Patienten (40,5\%) mit einem medianen Intervall von 9,0 Monaten (0,8 - 223,7 Monaten) zwischen der ersten SZT und dem ersten GK-MRT mit positivem ON-Nachweis mindestens eine ON. Bei einem Patienten waren ON erstmalig erst nach der zweiten SZT aufgetreten. Bei diesem Patienten wurde auch die erste SZT als Bezugspunkt gewählt. Zwischen der ersten und der zweiten SZT lag ein Jahr, in welchem eine GK-MRT-Untersuchung durchgeführt wurde, wo noch keine $\mathrm{ON}$ sichtbar waren.

\section{Alle Patienten $(n=105) \quad$ Patienten ohne SZT $(n=63) \quad$ Patienten mit SZT $(n=42)$}
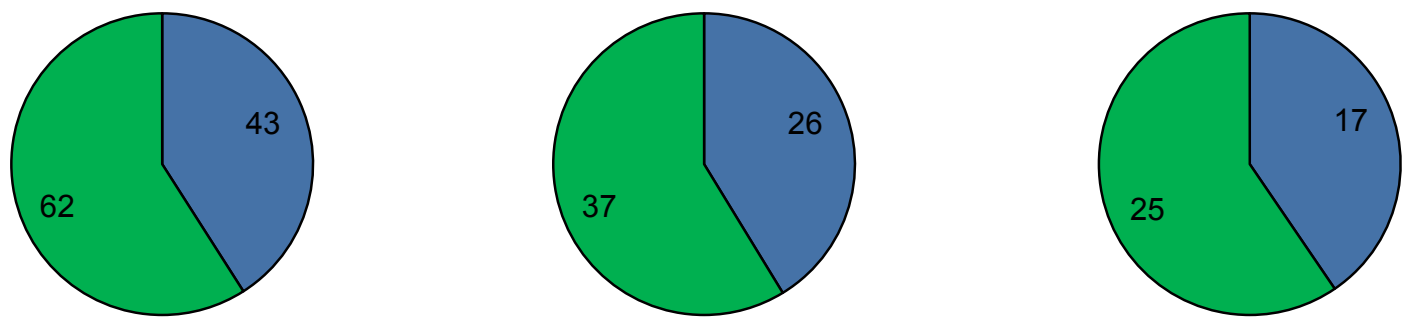

口Osteonekrosen vorhanden $\quad$ keine Osteonekrosen

Abbildung 7: Häufigkeit des Auftretens der Osteonekrosen in der GK-MRT je nach betrachteter Patientengruppe

Hinsichtlich des Spendertyps traten bei 3 der 12 Patienten mit einer autologen SZT $(25,0 \%)$ und bei 14 der 30 Kinder und Jugendlichen mit einer allogenen SZT $(46,7$ \%) ON auf. Bei einem Patienten mit initialer autologer SZT und nachfolgender allogener SZT wurden die ON bereits nach der ersten SZT nachgewiesen.

Für die Patienten ohne SZT wurde die Häufigkeit des Auftretens von ON in der GKMRT nach den jeweiligen Grunderkrankungen aufgeschlüsselt. ON traten nur bei Patienten mit ALL, Morbus Hodgkin, NHL und Osteosarkom auf. Die Häufigkeiten sind in Tabelle 7 zusammengetragen. 
Tabelle 7: Häufigkeiten des Auftretens der Osteonekrosen in der GK-MRT bei Patienten ohne SZT je nach Grunderkrankung

\begin{tabular}{|c|c|c|}
\hline Grunderkrankung & ON nachgewiesen & Keine ON \\
\hline ALL $(n=13)$ & $n=6(46,2 \%)$ & $n=7(53,8 \%)$ \\
\hline Morbus Hodgkin $(n=20)$ & $n=16(80 \%)$ & $n=4(20 \%)$ \\
\hline NHL $(n=5)$ & $n=3(60 \%)$ & $n=2(40 \%)$ \\
\hline Osteosarkom $(n=3)$ & $n=1(33,3 \%)$ & $n=2(66,7 \%)$ \\
\hline
\end{tabular}

Bei den insgesamt 43 Patienten mit nachgewiesenen ON in der GK-MRT klagten beim ersten positiven Nachweis 17 Mädchen und Jungen (39,5 \%) über Knochenschmerzen in mindestens einer Lokalisation der ON. 26 Kinder und Jugendliche $(60,5 \%)$ hatten keine Symptome.

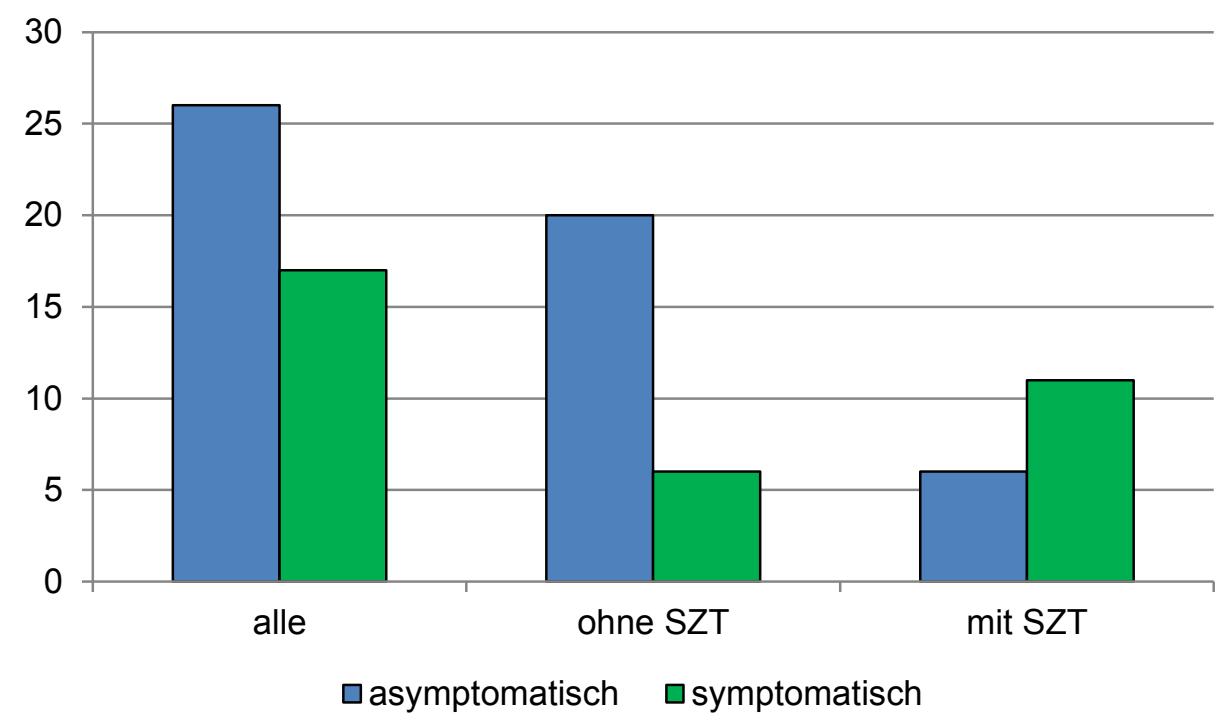

Abbildung 8: Aufteilung der Patienten mit Osteonekrosen in der GK-MRT bezüglich der Symptomatik

Aus Abbildung 8 ist ersichtlich, dass bei den Mädchen und Jungen ohne SZT zum Zeitpunkt des ersten positiven Nachweises von ON in der GK-MRT 20 Patienten asymptomatisch $(76,9 \%)$ und 6 Patienten symptomatisch $(23,1 \%)$ waren. Bei den Kindern und Jugendlichen mit SZT lag der Anteil der Patienten mit einer Schmerzsymptomatik bei $64,7 \%$ und ohne Symptomatik bei $54,5 \%$. 


\subsection{Lokalisationen der Osteonekrosen}

Für die Auswertung der Lokalisationen der $\mathrm{ON}$ wurden alle möglichen aufgetretenen ON unabhängig vom Zeitpunkt der Erstdetektion in einer GK-MRT-Aufnahme berücksichtigt. Die Analyse der 43 Patienten erfasste insgesamt 546 ON. Davon traten 268 Läsionen bei den Patienten ohne SZT und 278 ON bei den Patienten mit SZT auf.

\subsubsection{Anzahl und Verteilung der Lokalisationen der Osteonekrosen}

Die Ergebnisse zur Anzahl der verschiedenen Lokalisationen pro Patient sind in Tabelle 8 zusammengefasst.

Tabelle 8: Anzahl der Osteonekrosen pro Patient (SD = Standardabweichung)

\begin{tabular}{|c|c|c|c|c|}
\hline Patientengruppe & Minimum & Maximum & Mittelwert \pm SD & Median \\
\hline Alle Patienten & 1 & 49 & $12,7 \pm 11,0$ & 9,0 \\
\hline Patienten ohne SZT & 1 & 24 & $10,3 \pm 7,2$ & 8,5 \\
\hline Patienten mit SZT & 2 & 49 & $16,4 \pm 14,7$ & 14,0 \\
\hline
\end{tabular}

Wie in Abbildung 9 zu sehen, war in allen betrachteten Patientengruppen die untere Extremität häufiger als die obere Extremität von ON betroffen, unter allen Patienten bei 89,4 \%, unter den Patienten ohne SZT bei 94,4 \% und unter den Patienten mit SZT bei $84,5 \%$.

Alle Patienten mit 546 ON

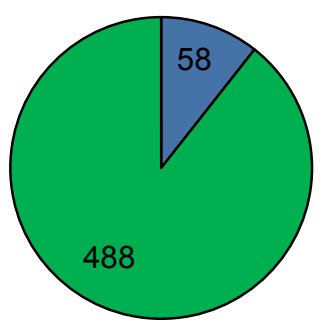

Patienten ohne SZT mit 268 ON

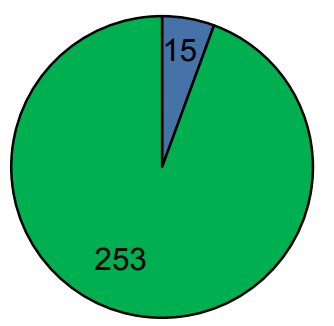

口obere Extremität $\quad$ uuntere Extremität

\section{Patienten mit SZT mit 278 ON}

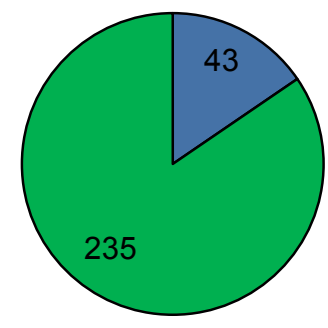

Abbildung 9: Aufteilung der Lokalisationen der $\mathrm{ON}$ in Abhängigkeit von den Extremitäten 
Aus Abbildung 10 wird ersichtlich, dass keine gravierenden Unterschiede hinsichtlich einer bevorzugt von ON betroffenen Körperseite bestanden.

Alle Patienten mit 546 ON

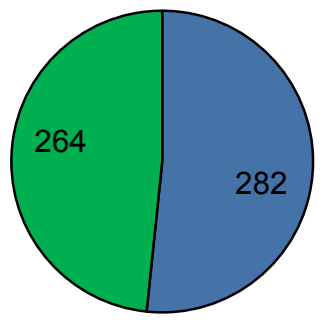

Patienten ohne SZT mit 268 ON

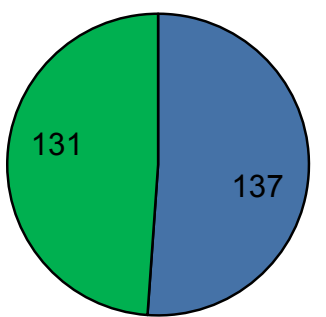

\section{Patienten mit SZT} mit 278 ON

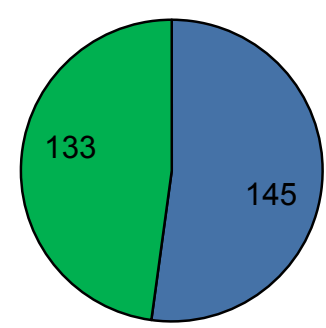

\section{$\square$ rechte Körperseite $\quad$ alinke Körperseite}

Abbildung 10: Aufteilung der Lokalisationen der $\mathrm{ON}$ in Abhängigkeit von der Körperseite

\subsubsection{Häufigkeit betroffener Knochen und gelenknaher Knochenregionen}

Da mehrere ON in einer Gelenkregion oder einem Knochen zusammengefasst wurden, schrumpfte die Gesamtzahl der ON auf insgesamt 276 Lokalisationen, wobei 141 ON bei den Patienten ohne SZT und 135 ON bei den Patienten mit SZT auftraten.

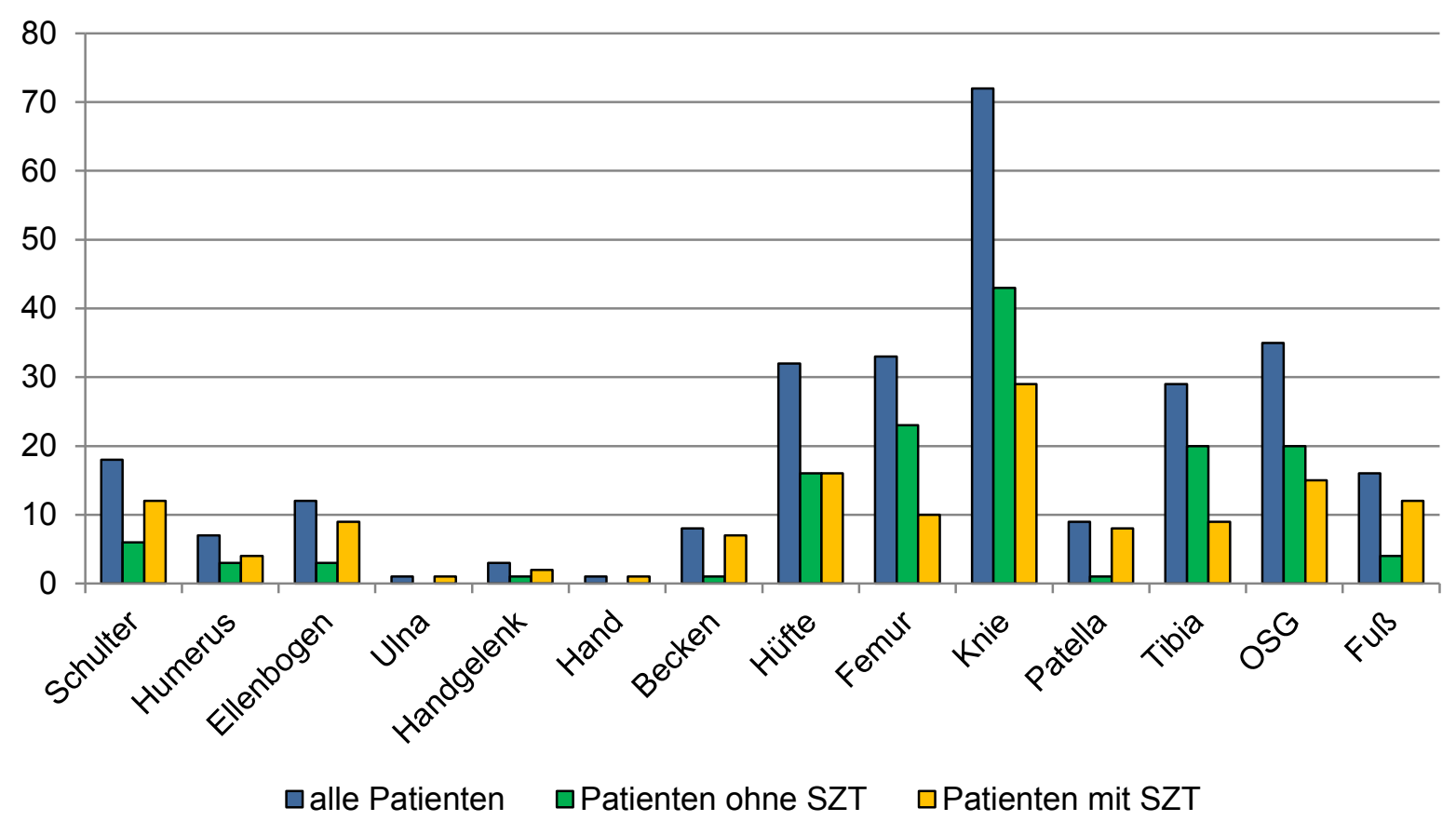

Abbildung 11: Anzahl der betroffenen Knochen und gelenknahen Knochenregionen durch Osteonekrosen aufgeteilt in die jeweiligen Patientengruppen 
Wie in Abbildung 11 erkennbar wurden $\mathrm{ON}$ am häufigsten in denen um das Kniegelenk liegenden Knochenbereichen detektiert. In Tabelle 9 sind die am häufigsten betroffenen Knochen und gelenknahen Knochenregionen in der unteren sowie oberen Extremität aufgeführt. Hier spiegelte sich auch die häufiger betroffene untere Extremität wider.

Tabelle 9: Häufigste Lokalisationen der Osteonekrosen aufgeteilt in die jeweiligen Patientengruppen

\begin{tabular}{|c|c|c|c|}
\hline Extremität & Alle Patienten & Patienten ohne SZT & Patienten mit SZT \\
\hline & Knie $(26,1 \%)$ & Knie $(30,5 \%)$ & \\
Untere & OSG $(12,7 \%)$ & Femur $(16,3 \%)$ & Knie $(21,5 \%)$ \\
Extremität & Femur $(12,0 \%)$ & Tibia $(14,2 \%)$ & Hüfte $(11,9 \%)$ \\
& Hüfte $(11,6 \%)$ & OSG $(14,2 \%)$ & OSG $(11,1 \%)$ \\
& Hüfte $(11,3 \%)$ & \\
\hline Obere & Schulter $(6,5 \%)$ & Schulter $(4,3 \%)$ & Schulter $(8,9 \%)$ \\
Extremität & \multicolumn{2}{|c}{} \\
\hline
\end{tabular}

Des Weiteren wurden die Anzahl der betroffenen gelenknahen Knochenregionen pro Patient für die jeweiligen Patientengruppen betrachtet (Tabelle 10).

Tabelle 10: Anzahl der von Osteonekrosen betroffenen Knochenbereiche um ein Gelenk pro Patient (SD = Standardabweichung)

\begin{tabular}{|c|c|c|c|c|}
\hline Patientengruppe & Minimum & Maximum & Mittelwert \pm SD & Median \\
\hline Alle Patienten & 0 & 11 & $4,0 \pm 2,9$ & 4,0 \\
\hline Patienten ohne SZT & 0 & 8 & $3,4 \pm 2,2$ & 3,5 \\
\hline Patienten mit SZT & 0 & 11 & $4,9 \pm 3,6$ & 4,0 \\
\hline
\end{tabular}




\subsubsection{Osteonekrosen in den das Kniegelenk bildenden Knochen}

Im Abschnitt 4.3.2 konnte gezeigt werden, dass die um das Kniegelenk liegenden Knochen am häufigsten von $\mathrm{ON}$ betroffen sind. Aus diesem Grund wurde jede einzelne ON, die sich mit Anteilen epiphysär oder metaphysär im distalen Femur, der proximalen Tibia oder der proximalen Fibula befand, in eine separate Auswertung eingeschlossen. 244 Läsionen konnten unter allen Patienten in diesen Lokalisationen nachgewiesen werden.

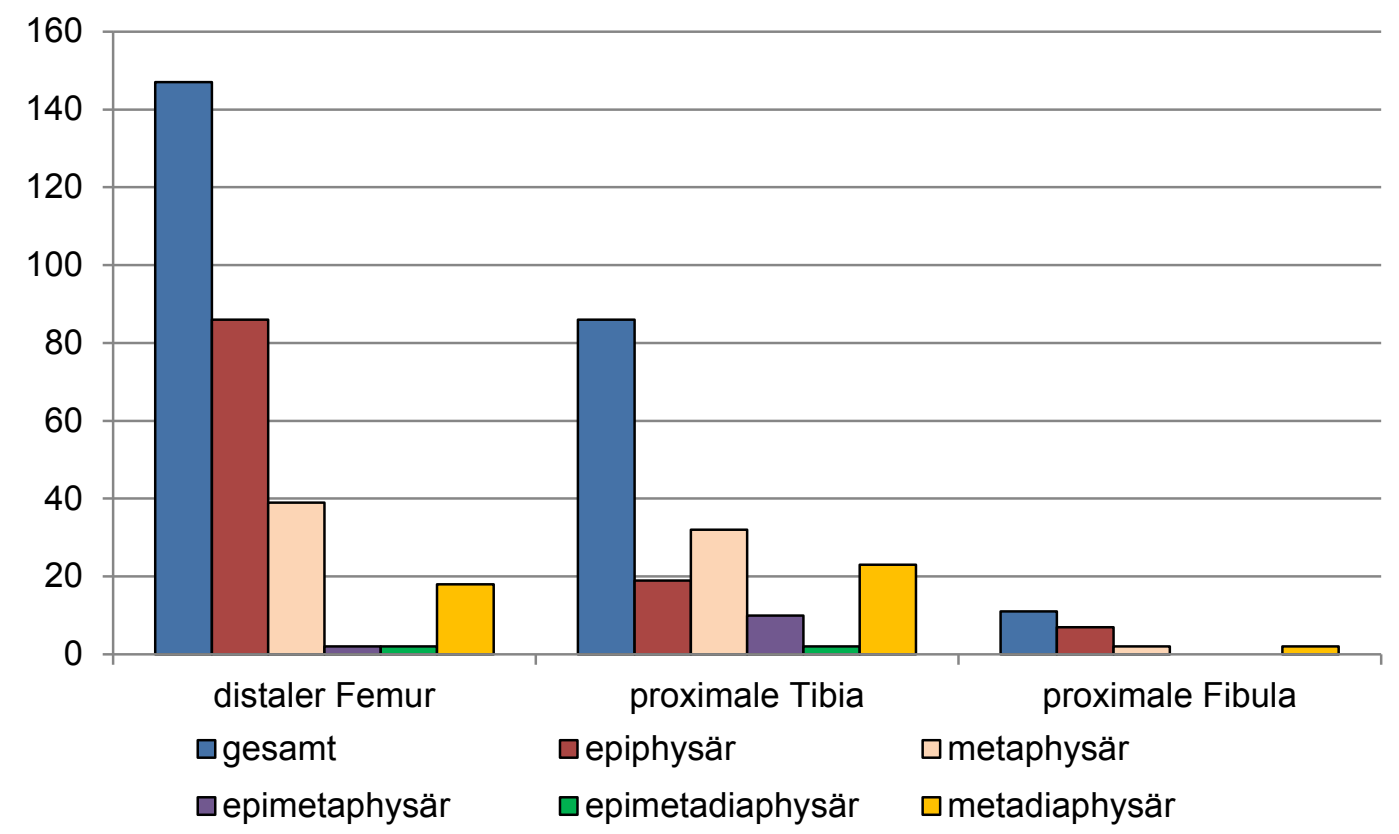

Abbildung 12: Verteilung der Osteonekrosen in den um das Kniegelenk liegenden Knochen

Die meisten ON in den um das Kniegelenk liegenden Knochen fanden sich mit 60,2 $\%$ im distalen Femur und dort vor allem in der Epiphyse (58,5\%). Metaphysär lagen $26,5 \%$ der Läsionen im distalen Femur. Am zweithäufigsten waren wie in Abbildung 12 ersichtlich die Nekrosen in der proximalen Tibia (35,2 \%) zu detektieren. Dort überwog der Anteil in der Metaphyse mit 37,2 \%. Aber auch metadiaphysär (26,7 \%) und epiphysär $(22,1 \%)$ konnte ein Großteil der ON nachgewiesen werden. 11 Nekrosen $(4,5 \%)$ waren in der proximalen Fibula zu sehen, dort vor allem epiphysär (63,6\%). 


\subsubsection{Lage der Osteonekrosen in den langen Röhrenknochen}

In den langen Röhrenknochen Humerus, Radius, Ulna, Femur, Tibia und Fibula fanden sich insgesamt 462 ON bei allen Patienten, aufgeteilt in 243 Läsionen bei den Patienten ohne SZT und 219 Nekrosen bei den Patienten mit SZT.

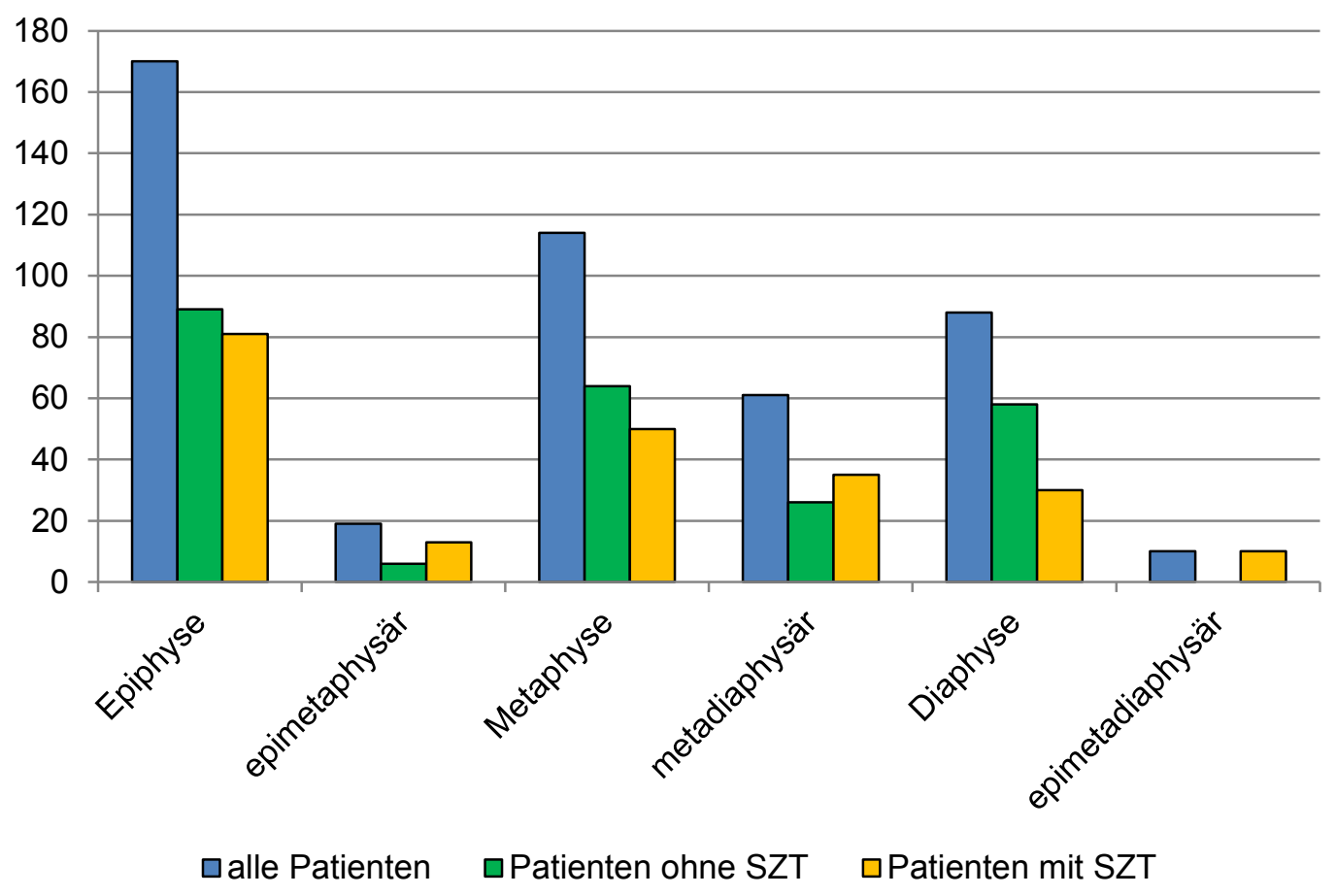

Abbildung 13: Anzahl der Osteonekrosen in der jeweiligen Lage in den langen Röhrenknochen aufgeteilt in die jeweiligen Patientengruppen

Wie in Abbildung 13 ersichtlich war am häufigsten die Epiphyse der langen Röhrenknochen von ON betroffen. Der Anteil lag unter allen Patienten bei 36,8 \%, bei den Kindern und Jugendlichen ohne SZT bei 36,6 \% und bei den Mädchen und Jungen mit SZT bei 37,0 \%. Am zweithäufigsten war die Metaphyse in allen drei betrachteten Patientengruppen mit Häufigkeiten von $24,7 \%$ unter allen Patienten, $26,3 \%$ unter den Patienten ohne SZT und 22,8 \% unter den Patienten mit SZT von ON betroffen. 
Die Gelenkflächenbeteiligung wurde bei allen ON beurteilt, welche Anteil an der Epiphyse hatten, d.h. die epiphysären, epimetaphysären sowie epimetadiaphysären Läsionen. Die Anzahl der diesem Kriterium entsprechenden ON betrug insgesamt 199, aufgeteilt in 95 Läsionen bei den Patienten ohne SZT und 104 Läsionen bei den Patienten mit SZT. Dabei wurde unabhängig vom Ausmaß lediglich betrachtet, ob die ON die Gelenkfläche erreichen oder nicht.

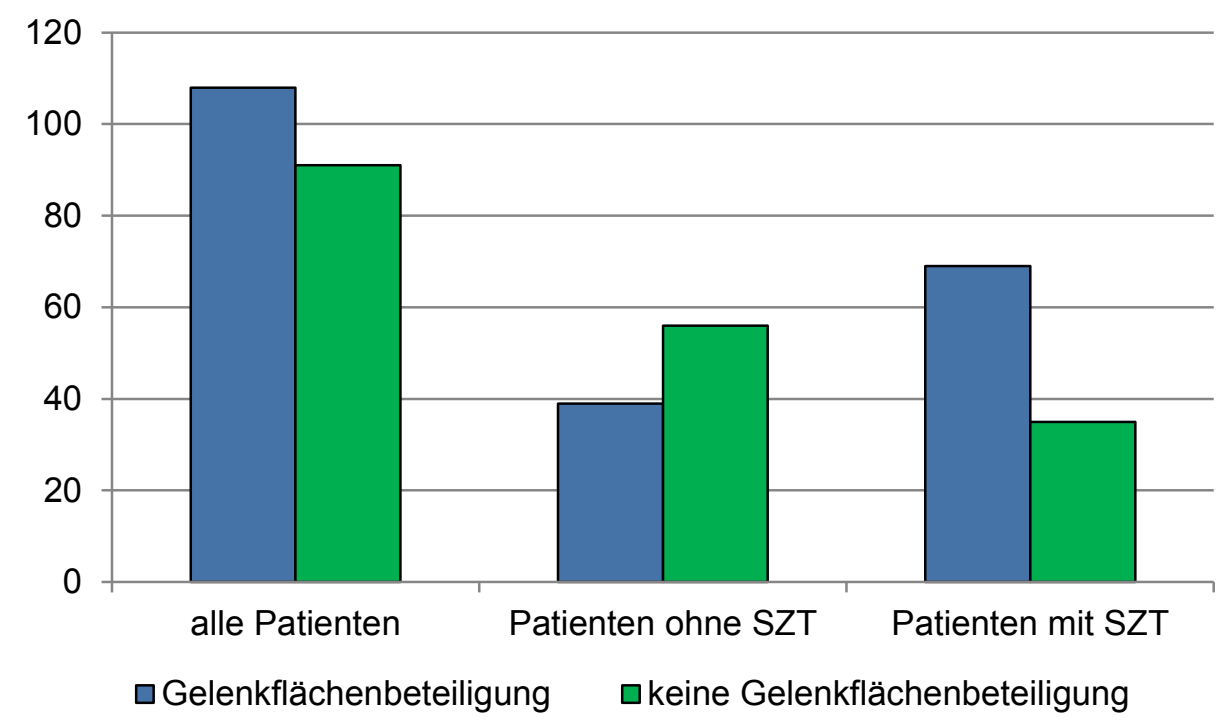

Abbildung 14: Anzahl der Osteonekrosen mit Anteil an der Epiphyse mit und ohne Gelenkflächenbeteiligung aufgeteilt in die jeweiligen Patientengruppen

In Abbildung 14 ist zu sehen, dass in etwa genauso viele ON mit Anteil an der Epiphyse die Gelenkfläche erreichten (54,3 \%) sowie diese nicht in Mitleidenschaft zogen (45,7\%). Die Gelenkflächenbeteiligung dominierte unter den ON der Patienten mit SZT (66,3 \%). Bei den Patienten ohne SZT überwogen die ON, welche die Gelenkfläche nicht erreichten (58,9\%).

In der Analyse der Häufigkeit der Gelenkflächenbeteiligung getrennt nach der oberen und unteren Extremität konnte bei den $34 \mathrm{ON}$ der oberen Extremität, welche Anteil an der Epiphyse zeigten, mit 55,9 \% kein Erreichen der Gelenkfläche verifiziert werden. Bei den 165 Läsionen der unteren Extremität mit Anteil an der Epiphyse war in $56,4 \%$ die Gelenkfläche der Epiphyse mit in die ON einbezogen. 


\subsection{Einflussfaktoren auf das Auftreten von Osteonekrosen}

Der Einflussfaktor Alter bei Diagnose der Grunderkrankung (Patienten ohne SZT) bzw. bei der ersten SZT (Patienten mit SZT) sowie der Einflussfaktor Geschlecht wurden graphisch anhand von Kaplan-Meier-Kurven dargestellt, bei denen zeitabhängig ab der Diagnosestellung der Grunderkrankung bzw. der ersten SZT die Wahrscheinlichkeit des Auftretens von ON in der GK-MRT für die jeweiligen Patientengruppen abgebildet wurde. Dabei entsprach ein Ereignis dem ersten Nachweis von ON in der GK-MRT bei einem Patienten. Ebenfalls wurde mit dieser Methode der Einfluss der Höhe der gegebenen kumulativen Steroiddosis sowie der Art des verabreichten Steroids auf das Auftreten von ON in der GK-MRT beurteilt.

\subsubsection{Einflussfaktor Alter}

Unter den 63 Patienten ohne SZT fanden sich in der Altersgruppe $\leq 10$ Jahre bei der Diagnose der jeweiligen Grunderkrankung 27 Patienten (42,9 \%). Demzufolge waren 36 Patienten in dieser Gruppe $(57,1 \%)$ älter als 10 Jahre.

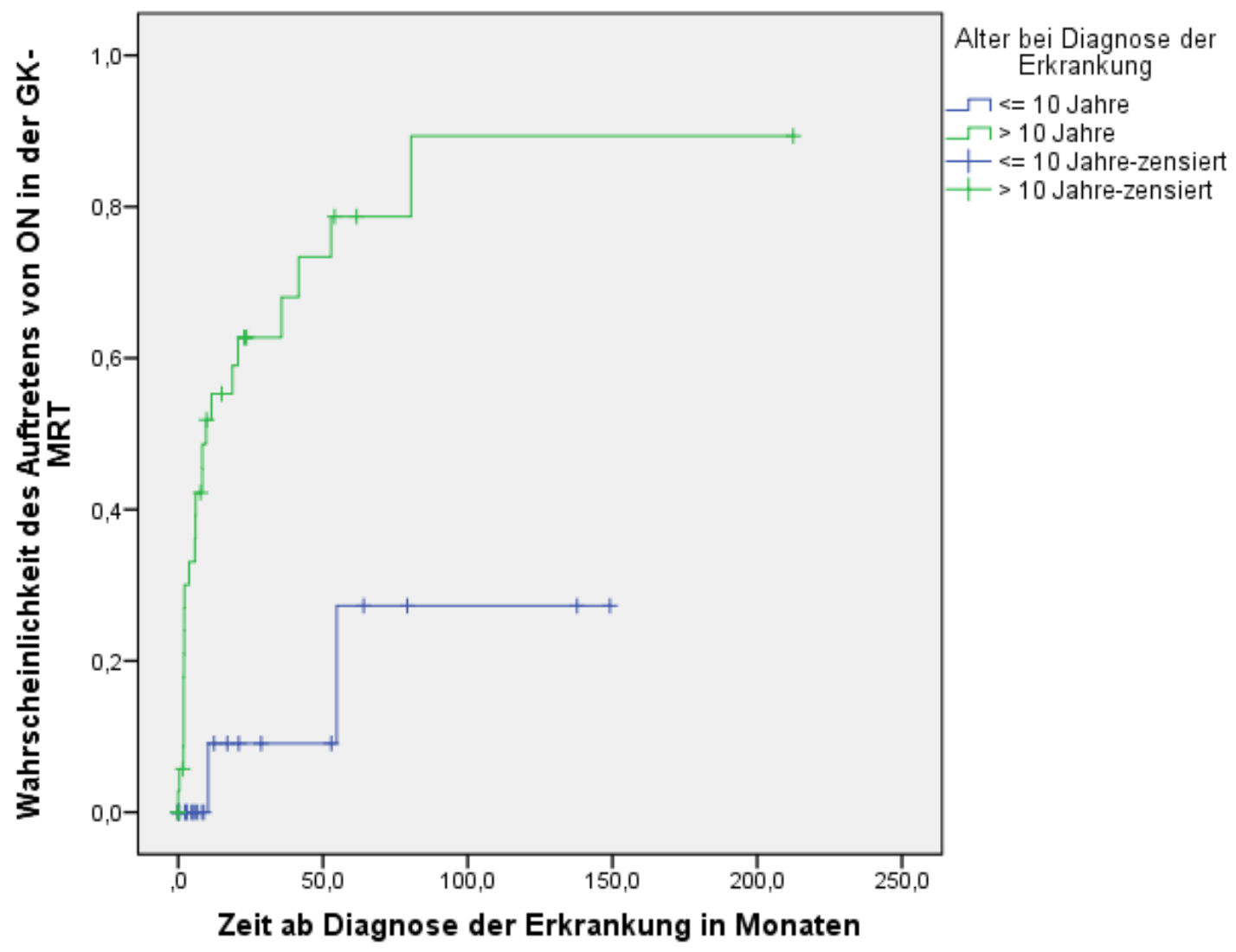

Abbildung 15: Kaplan-Meier-Kurven zur Darstellung des Einflussfaktors Alter bei der Diagnose der Grunderkrankung auf das Auftreten von Osteonekrosen in der GK-MRT bei Patienten ohne SZT 
Bei den Kindern $\leq 10$ Jahre traten bei 2/27 Patienten $(7,4 \%)$ ON in der GK-MRT auf. Unter den Patienten mit einem Alter > 10 Jahre bei Diagnosestellung der Grunderkrankung wurden bei $24 / 36$ Patienten $(66,7 \%)$ in der Bildgebung ON nachgewiesen. Wie in Abbildung 15 ersichtlich steigt der Graph der Patienten der Altersgruppe $\leq 10$ Jahre weniger steil an als der Graph der Patienten $>10$ Jahre. Daraus ergibt sich, dass die älteren Patienten ein höheres Risiko für ein Auftreten von ON in der GK-MRT aufweisen. Im Log-Rank-Test wurde dieses Ergebnis mit $\mathrm{p}<$ 0,001 als statistisch signifikant bestätigt. Die mediane Zeit, bei welcher $50 \%$ der Patienten $\leq 10$ Jahre ON in der GK-MRT aufwiesen, konnte nicht abgelesen werden, da der Graph dieser Patientengruppe diese Bedingung nicht erreicht. Es kann jedoch abgeschätzt werden, dass 55 Monate nach der Diagnosestellung der Grunderkrankung bei 27,8 \% der Kinder $\leq 10$ Jahre und bei 78,7 \% der Patienten > 10 Jahre ON in der GK-MRT aufgetreten waren.

Auch in der Cox-Regression fand sich ein statistisch signifikantes Ergebnis ( $\mathrm{p}=$ 0,002). Die Patienten mit einem Alter von > 10 Jahre bei der Diagnosestellung der Grunderkrankung unterlagen einem 9,6-mal größeren Risiko für einen positiven Nachweis von ON in der GK-MRT als die Kinder $\leq 10$ Jahre (Hazard Ratio $(H R)=$ 9,614). 
Die 42 Patienten mit SZT teilten sich in 18 Kinder (42,9\%) mit einem Alter von $\leq 10$ Jahren bei der ersten SZT und 24 Patienten $(57,1 \%)$ mit einem Alter von > 10 Jahren auf.

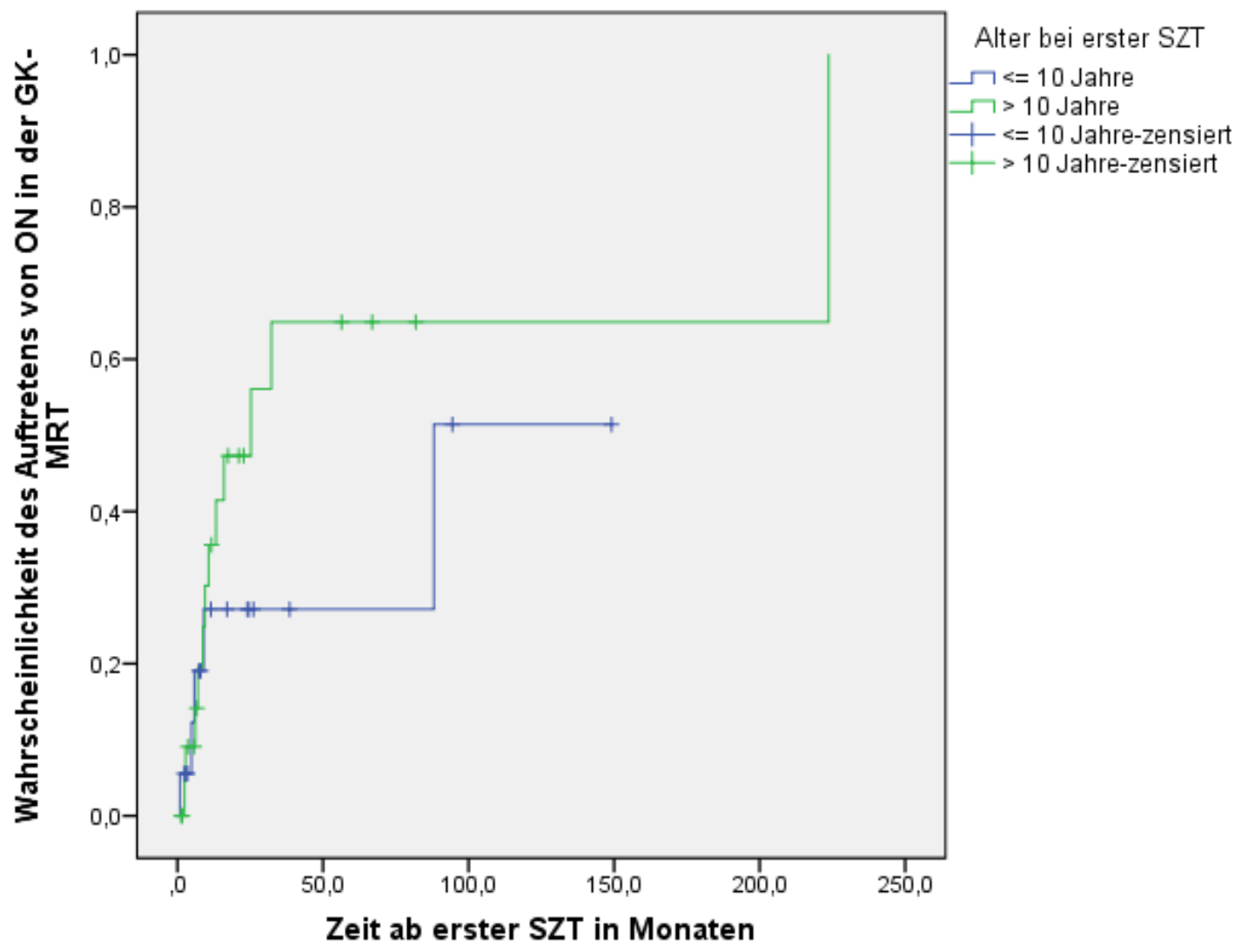

Abbildung 16: Kaplan-Meier-Kurven zur Darstellung des Einflussfaktors Alter bei der ersten SZT auf das Auftreten von Osteonekrosen in der GK-MRT bei Patienten mit SZT

Unter den Patienten mit SZT, die bei der ersten SZT $\leq 10$ Jahre alt waren, traten bei 5/18 Kindern und Jugendlichen (27,8 \%) ON in der GK-MRT auf. Bei den älteren Patienten mit einem Alter von > 10 Jahren waren es 12/24 Jungen und Mädchen $(50,0 \%)$, bei denen $\mathrm{ON}$ in der Bildgebung nachgewiesen werden konnten. Die beiden Graphen in Abbildung 16 zeigen zu Beginn einen ähnlichen Verlauf. Danach steigt der Graph der Patienten der Altersgruppe > 10 Jahre tendenziell steiler an, was ein größeres Risiko für ein Auftreten von ON in der GK-MRT bei den älteren Patienten bedeutet. Die mediane Zeit, bei welcher bei der Hälfte der Patienten in den jeweiligen Altersgruppen ON in der GK-MRT nachgewiesen wurden, wird auf 88,1 Monate für die Kinder $\leq 10$ Jahre und auf 25,3 Monate für die Patienten > 10 Jahre geschätzt. Daraus kann auch in dieser Patientengruppe auf einen deutlich früheren ON-Nachweis in der GK-MRT bei den älteren Patienten geschlossen werden. Diese 
Aussagen waren mit $p=0,287$ im Log-Rank-Test statistisch allerdings nicht signifikant.

In der Cox-Regression ergab sich eine Hazard Ratio von HR = 1,779. Das bedeutet, dass Patienten mit SZT in der Altersgruppe > 10 Jahre bei der ersten SZT im Vergleich zu der Gruppe $\leq 10$ Jahre einem 1,8-mal höherem Risiko für ein Auftreten von ON in der GK-MRT unterlagen. Dieses Ergebnis war ebenfalls statistisch nicht signifikant $(p=0,293)$.

\subsubsection{Einflussfaktor Geschlecht}

36 Jungen $(57,1 \%)$ und 27 Mädchen (42,9 \%) bildeten die Patientengruppe ohne SZT.

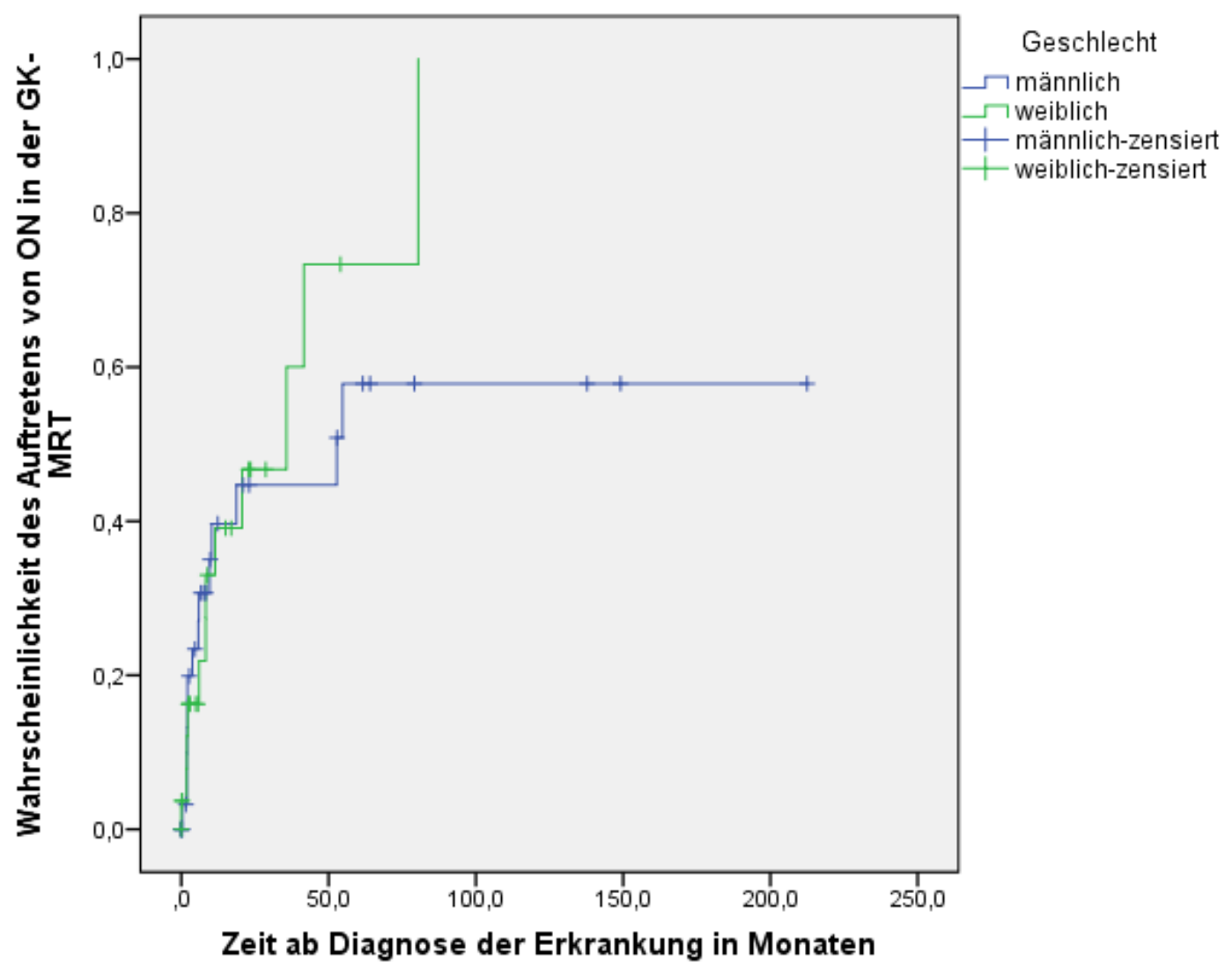

Abbildung 17: Kaplan-Meier-Kurven zur Darstellung des Einflussfaktors Geschlecht auf das Auftreten von Osteonekrosen in der GK-MRT bei Patienten ohne SZT

Bei 14/36 Jungen (38,9 \%) und 12/27 Mädchen (44,4 \%), die sich keiner SZT unterziehen mussten, konnten in der GK-MRT ON nachgewiesen werden. In Abbildung 17 ist bis ca. zwei Jahre nach Diagnosestellung ein ähnlicher Verlauf der Graphen der männlichen und weiblichen Patienten sichtbar. Die Kurve der Mädchen steigt danach steiler an, was eine Tendenz für ein größeres Risiko für das Auftreten 
von ON in der GK-MRT bei weiblichen Patienten darstellt. Die mediane Zeit, bei welcher bei $50 \%$ der betrachteten Patienten ON in der GK-MRT nachgewiesen werden konnten, wird auf 52,8 Monate für die Jungen und auf 35,7 Monate für die Mädchen geschätzt. Daraus kann geschlossen werden, dass die weiblichen Patienten eventuell früher ON erleiden. Der Unterschied war im Log-Rank-Test statistisch nicht signifikant ( $p=0,474)$.

In der Cox-Regression fand sich eine Hazard Ratio von $H R=1,33$, was ein 1,3-mal größeres Risiko für ein Auftreten von ON in der GK-MRT bei Mädchen ohne SZT im Vergleich zu Jungen bedeutete. Dieses Ergebnis war ebenfalls statistisch nicht signifikant $(p=0,477)$.

Die 42 Patienten mit SZT teilten sich in 26 männliche $(61,9 \%)$ und 16 weibliche $(38,1 \%)$ Kinder und Jugendliche.

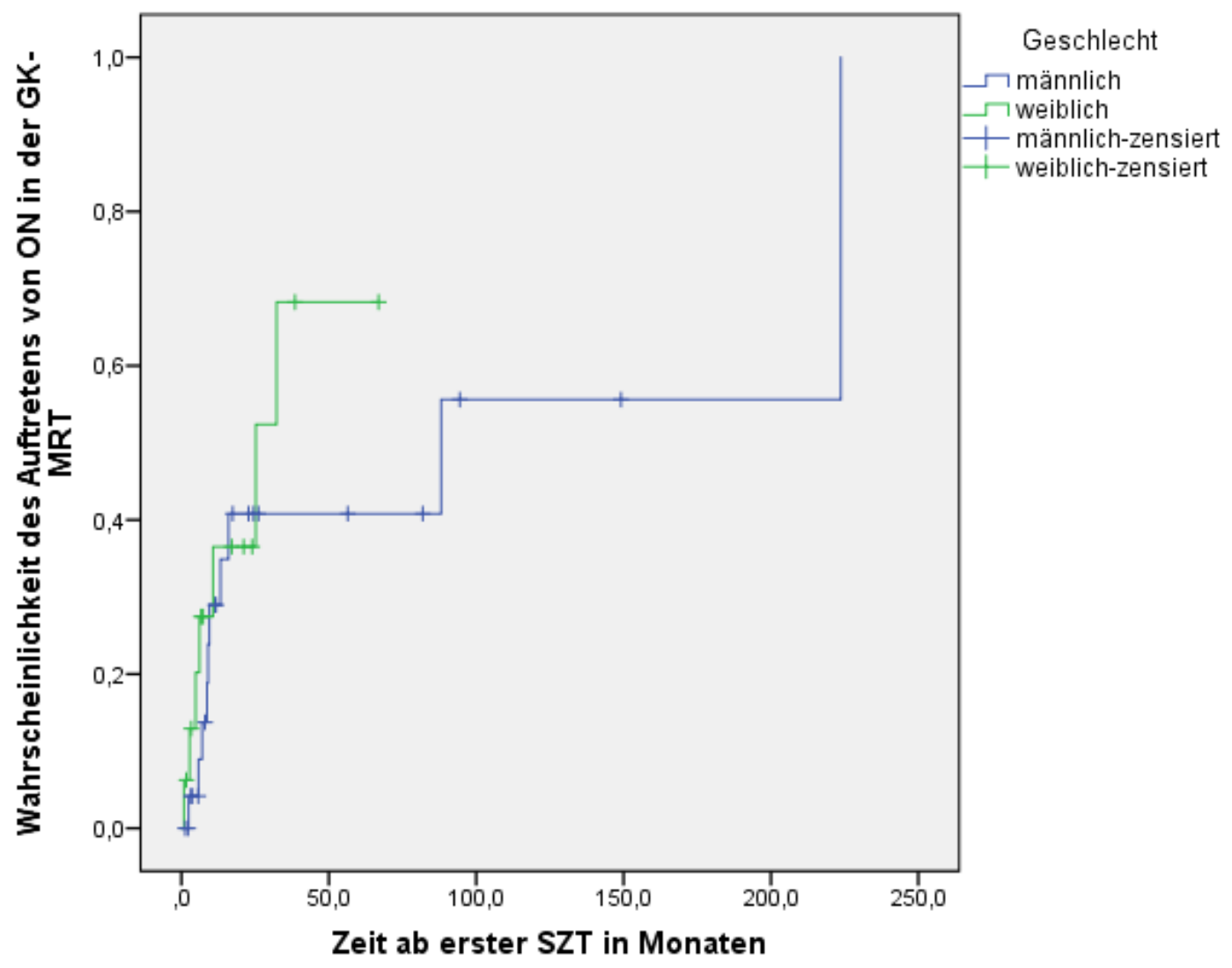

Abbildung 18: Kaplan-Meier-Kurven zur Darstellung des Einflussfaktors Geschlecht auf das Auftreten von Osteonekrosen in der GK-MRT bei Patienten mit SZT

Unter den Patienten mit SZT traten ON in der GK-MRT bei 10/16 Mädchen (38,5 \%) und $7 / 26$ Jungen (43,8 \%) auf. Wie in Abbildung 18 ersichtlich, ähneln sich die 
Kurven der männlichen und weiblichen Patienten mit SZT überwiegend. Im Verlauf steigt der Graph der Mädchen etwa zwei Jahre nach der SZT steiler an. Daraus ist zu erkennen, dass es auch bei den Patienten mit SZT eine Tendenz zum größeren Risiko für ein Auftreten von ON bei weiblichen Patienten gab. Die mediane Zeit, in welcher bei der Hälfte der betrachteten Patienten nach SZT ON in der GK-MRT aufgetreten waren, wurde für die Jungen auf 88,1 Monate und für die Mädchen auf 25,3 Monate geschätzt. Somit konnte auch hier gesagt werden, dass bei den weiblichen Patienten möglicherweise ON früher auftreten. Diese Aussagen waren laut dem Log-Rank-Test statistisch nicht signifikant $(p=0,374)$.

Mit einer Hazard Ratio von HR = 1,578 in der Cox-Regression traten die ON in der GK-MRT mit einem 1,6-mal höheren Risiko bei Mädchen als bei Jungen nach einer SZT auf. Dieses Ergebnis war mit $p=0,379$ ebenfalls statistisch nicht signifikant.

\subsubsection{Einflussfaktor Art des verabreichten Steroids und Höhe der kumulativen}

\section{Steroiddosis}

Für alle Patienten ohne SZT wurde die verabreichte kumulative Steroiddosis bestimmt.

Für die Betrachtung des Einflusses der Gesamtsteroiddosis auf das Auftreten von ON in der GK-MRT wurden 63 Patienten eingeschlossen. In Tabelle 11 ist die Anzahl der Patienten in den jeweiligen Gruppen zusammengefasst.

Tabelle 11: Anzahl der Patienten in den jeweiligen Patientengruppen nach Höhe der verabreichten kumulativen Steroiddosis

\begin{tabular}{|c|c|c|}
\hline Kumulative Steroiddosis & $\begin{array}{c}\text { Anzahl Patienten } \\
(\mathbf{n}=\mathbf{6 3})\end{array}$ & $\begin{array}{c}\text { Patienten mit ON } \\
(\mathbf{n}=\mathbf{2 6})\end{array}$ \\
\hline $0 \mathrm{~g} / \mathrm{m}^{2}$ & 23 & $1(4,3 \%)$ \\
\hline$\leq 2,8 \mathrm{~g} / \mathrm{m}^{2}$ & 18 & $13(72,2 \%)$ \\
\hline$>2,8 \mathrm{~g} / \mathrm{m}^{2}$ & 22 & $12(54,5 \%)$ \\
\hline
\end{tabular}

Die mediane verabreichte kumulative Steroiddosis der 40 Patienten, welche Prednisolon und/oder Dexamethason erhielten, lag bei 3,0 g/m² $\left(1,0-7,7 \mathrm{~g} / \mathrm{m}^{2}\right)$. 


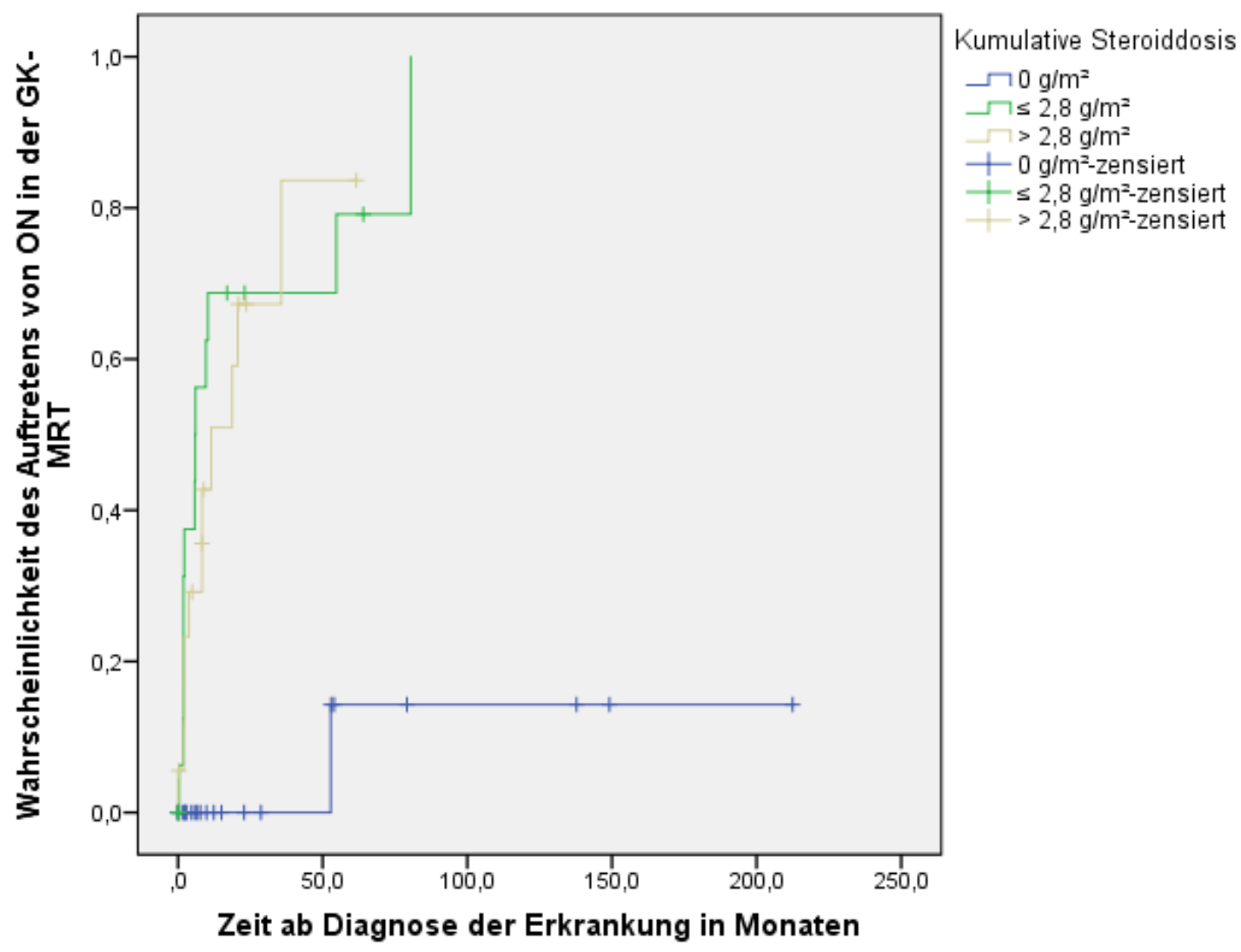

Abbildung 19: Kaplan-Meier-Kurven zur Darstellung des Einflussfaktors Höhe der kumulativen Steroiddosis auf das Auftreten von Osteonekrosen in der GK-MRT bei Patienten ohne SZT

Abbildung 19 stellt die unterschiedlichen Verläufe der Graphen pro Patientengruppe dar. Die Nullhypothese auf Gleichheit der Kurven konnte mit $p<0,001$ aus dem LogRank-Test abgelehnt werden. Das Ergebnis war somit statistisch signifikant. Es ist deutlich zu sehen, dass der Graph der Patienten, welche keine Steroide verabreicht bekamen, flacher verläuft und diese Patienten somit ein niedrigeres Risiko aufzeigten, im Verlauf ON zu entwickeln, die mit der GK-MRT detektiert werden konnten. Die mediane Zeit, nach der 50 \% der Patienten in einer Gruppe ON in der GK-MRT aufwiesen, konnte nicht über alle Kurven verglichen werden, da in der Gruppe mit einer Steroiddosis von $0 \mathrm{~g} / \mathrm{m}^{2}$ diese Bedingung nicht erreicht wurde. Für die Gruppe mit der niedrigeren Gesamtsteroiddosis wurde diese mediane Zeit auf 5,9 Monate und für die Patienten mit einer Dosis von $>2,8 \mathrm{~g} / \mathrm{m}^{2}$ auf 18,7 Monate geschätzt. Daraus ist zu erkennen, dass möglicherweise die ON in der GK-MRT bei den Patienten mit der niedrigeren Dosis an Steroiden früher auftraten. Eine statistische Signifikanz wurde für diese Aussage nicht beobachtet. 
In der Cox-Regression wurden die Gruppen ohne und mit Steroide verglichen, wobei die Höhe der Dosis in der Gruppe mit Steroiden nicht berücksichtigt wurde. Mit einer Hazard Ratio von HR = 21,62 hatten die Patienten, die Steroide im Rahmen ihrer Chemotherapie erhielten, im Vergleich zu jenen, die keine Steroide verabreicht bekamen, ein 21,6-fach höheres Risiko, dass ON in der GK-MRT auftraten. Dieses Ergebnis war mit $p=0,003$ statistisch signifikant.

Zusätzlich wurde mittels der Cox-Regression ein Vergleich zwischen der Patientengruppe mit der niedrigeren Gesamtsteroiddosis und jener Gruppe mit der höheren Dosis durchgeführt. Die ausgegebene Hazard Ratio betrug $H R=0,795$. Somit hatten die Patienten mit der niedrigeren Steroiddosis ein höheres Risiko des positiven Nachweises von ON in der GK-MRT. Dieses Ergebnis war mit $p=0,579$ statistisch nicht signifikant.

Zur Beurteilung, ob ein einzelnes Steroid oder die Kombination von zwei verschiedenen Präparaten ein höheres Risiko für das Auftreten von ON in der GKMRT mit sich bringt, wurden 40 Patienten, die im Rahmen ihrer Chemotherapie Steroide erhielten, in den Vergleich eingeschlossen. In Tabelle 12 sind die Patientengruppen dargestellt.

Tabelle 12: Anzahl der Patienten in den jeweiligen Patientengruppen in Abhängigkeit von der Art des verabreichten Steroids

\begin{tabular}{|c|c|c|}
\hline Art des Steroids & Anzahl Patienten & Patienten mit ON \\
\hline Dexamethason & 5 & $2(40,0 \%)$ \\
\hline Prednisolon & 21 & $16(76,2 \%)$ \\
\hline Dexamethason + Prednisolon & 14 & $7(50,0 \%)$ \\
\hline
\end{tabular}

Tabelle 13: Vergleich des Auftretens von ON in der GK-MRT in Abhängigkeit von der Art des verabreichten Steroids

\begin{tabular}{|c|c|c|}
\hline Vergleich Art des Steroids & Hazard Ratio & Signifikanz $\mathbf{p}$ \\
\hline Kombination vs. einzelnes Steroid & 2,749 & 0,038 \\
\hline Prednisolon vs. Dexamethason & 0,715 & 0,657 \\
\hline
\end{tabular}


In der Cox-Regression wurde die Patientengruppe, die sowohl Prednisolon als auch Dexamethason bekam, mit einer Patientengruppe, die nur ein Steroid erhielt, verglichen. Die Zuteilung in die zweite Gruppe erfolgte unabhängig von dem verabreichten Steroid. Wie in Tabelle 13 ersichtlich unterlagen die Patienten, welche ein einzelnes Steroid erhielten, einem signifikant 2,7-fach höheren Risiko, dass in der GK-MRT ON detektierbar waren, als die Patienten mit einer Kombination.

Ebenfalls konnte gezeigt werden, dass die Patienten, die nur Prednisolon erhielten, ein höheres Risiko für das Auftreten von ON in der GK-MRT besaßen als jene Kinder und Jugendliche, die ausschließlich Dexamethason als Steroid im Rahmen ihrer Chemotherapie bekamen. Dieses Ergebnis war statistisch nicht signifikant.

In dieser Auswertung waren die Patientenzahlen $(n=40)$ allerdings sehr klein, um aussagekräftige Ergebnisse zu erhalten. Vor allem die Patientengruppe, die nur Dexamethason verabreicht bekam, stellte mit 5 Patienten keine repräsentative Gruppe dar.

Im Folgenden werden die Steroide Prednisolon und Dexamethason getrennt betrachtet. Es sollte dargestellt werden, ob die Höhe der verabreichten Dosis eines Steroids einen Einfluss auf das Auftreten von ON in der GK-MRT aufweist. Dabei muss beachtet werden, dass jene Patienten, welche $0 \mathrm{~g} / \mathrm{m}^{2}$ von einem der beiden Steroide bekamen, nicht gleichzeitig gar keine Steroide erhielten. Das betraf die Patienten, die in der vorherigen Betrachtung nur Prednisolon ( $n=21$ ) oder nur Dexamethason $(n=5)$ verabreicht bekamen. Von dem jeweils anderen Steroid erhielten sie eine Dosis von $0 \mathrm{~g} / \mathrm{m}^{2}$.

Zunächst erfolgt die Auswertung bezüglich der Höhe der Prednisolondosis. Es wurden die 63 Patienten ohne SZT betrachtet.

Tabelle 14: Anzahl der Patienten in den jeweiligen Patientengruppen nach Höhe der verabreichten Prednisolondosis

\begin{tabular}{|c|c|c|}
\hline Dosis Prednisolon & Anzahl Patienten & Patienten mit ON \\
\hline $0 \mathrm{~g} / \mathrm{m}^{2}$ & 28 & $3(10,7 \%)$ \\
\hline$\leq 2,0 \mathrm{~g} / \mathrm{m}^{2}$ & 20 & $11(55,0 \%)$ \\
\hline$>2,0 \mathrm{~g} / \mathrm{m}^{2}$ & 15 & $12(80,0 \%)$ \\
\hline
\end{tabular}


Die mediane verabreichte Prednisolondosis der 35 Patienten, welche dieses Steroid erhielten, betrug $1,8 \mathrm{~g} / \mathrm{m}^{2}\left(0,3-4,2 \mathrm{~g} / \mathrm{m}^{2}\right)$.

Tabelle 15: Vergleich des Auftretens von ON in der GK-MRT in Abhängigkeit von der Höhe der verabreichten Prednisolondosis

\begin{tabular}{|c|c|c|}
\hline Vergleich Prednisolondosis & Hazard Ratio & Signifikanz $\mathbf{p}$ \\
\hline $0 \mathrm{~g} / \mathrm{m}^{2}$ vs. $>0 \mathrm{~g} / \mathrm{m}^{2}$ & 7,589 & 0,001 \\
\hline$\leq 2,0 \mathrm{~g} / \mathrm{m}^{2}$ vs. $>2,0 \mathrm{~g} / \mathrm{m}^{2}$ & 1,563 & 0,304 \\
\hline
\end{tabular}

In der Tabelle 15 sind die Ergebnisse der Cox-Regression aufgeführt. Daran ist zu erkennen, dass die Kinder und Jugendliche, welche Prednisolon verabreicht bekamen, ein 7,6-mal höheres Risiko des ON-Auftretens in der GK-MRT aufwiesen als jene Patienten, die kein Prednisolon erhielten. Dieses Ergebnis war statistisch signifikant $(p=0,001)$.

In einem Vergleich der Patientengruppe mit der niedrigeren zu der Gruppe mit der höheren Prednisolondosis konnte ein 1,6-mal höheres Risiko bei der Patientengruppe mit einer Dosis von $>2,0 \mathrm{~g} / \mathrm{m}^{2}$ ermittelt werden. Dieses Ergebnis war statistisch nicht signifikant $(p=0,304)$.

Im Nachfolgenden wird das Risiko für das Auftreten von ON in der GK-MRT für 63 Patienten ohne SZT bezüglich der Höhe der verabreichten Dexamethasondosis ausgewertet.

Tabelle 16: Anzahl der Patienten in den jeweiligen Patientengruppen nach Höhe der verabreichten Dexamethasondosis

\begin{tabular}{|c|c|c|}
\hline Dosis Dexamethason & Anzahl Patienten & Patienten mit ON \\
\hline $0 \mathrm{~g} / \mathrm{m}^{2}$ & 44 & $17(38,6 \%)$ \\
\hline$\leq 0,3 \mathrm{~g} / \mathrm{m}^{2}$ & 10 & $3(30,0 \%)$ \\
\hline$>0,3 \mathrm{~g} / \mathrm{m}^{2}$ & 9 & $6(66,7 \%)$ \\
\hline
\end{tabular}

Die mediane verabreichte Dosis an Dexamethason bei den 19 Patienten, die dieses Steroid erhielten, lag bei $0,3 \mathrm{~g} / \mathrm{m}^{2}\left(0,2-0,9 \mathrm{~g} / \mathrm{m}^{2}\right)$. 
Tabelle 17: Vergleich des Auftretens von ON in der GK-MRT in Abhängigkeit von der Höhe der verabreichten Dexamethasondosis

\begin{tabular}{|c|c|c|}
\hline Vergleich Dexamethasondosis & Hazard Ratio & Signifikanz $\mathbf{p}$ \\
\hline $0 \mathrm{~g} / \mathrm{m}^{2}$ vs. $>0 \mathrm{~g} / \mathrm{m}^{2}$ & 1,212 & 0,642 \\
\hline$\leq 0,3 \mathrm{~g} / \mathrm{m}^{2}$ vs. $>0,3 \mathrm{~g} / \mathrm{m}^{2}$ & 0,713 & 0,681 \\
\hline
\end{tabular}

In Tabelle 17 sind die Ergebnisse der Cox-Regression ersichtlich. Die Patienten, welche Dexamethason verabreicht bekamen, unterlagen einem 1,2-fach höheren Risiko für ein Auftreten von $\mathrm{ON}$ in der GK-MRT als jene Patienten ohne Dexamethason. Dieses Ergebnis war statistisch nicht signifikant.

Im Vergleich zwischen der Patientengruppe mit der niedrigeren Dexamethasondosis und jener Gruppe mit der höheren verabreichten Dosis zeigte sich, dass die Patienten mit der niedrigeren Dosis ein gering erhöhtes Risiko des ON-Auftretens in der GK-MRT aufwiesen. Dieses Ergebnis war statistisch nicht signifikant. In diesen Vergleich wurden nur sehr wenige Patienten $(n=19)$ einbezogen, wodurch das Ergebnis nicht sehr aussagekräftig war.

\subsection{Zeitlicher Verlauf der Osteonekrosen bei Patienten mit Morbus Hodgkin}

8 Jungen und 7 Mädchen mit Morbus Hodgkin als Grunderkrankung entwickelten in der GK-MRT nachweisbare ON, die im weiteren Verlauf in mehreren Bildgebungen über eine mediane Beobachtungsdauer von 18,8 Monaten (3,3 - 80,2 Monate) beurteilt wurden. Das mediane Alter bei der Diagnosestellung des Grundleidens lag bei 16,1 Jahren. Nach einer medianen Zeit von 2,2 Monaten (0,0 - 35,7 Monate) nach Diagnosestellung der Grunderkrankung traten in der GK-MRT erstmals ON auf. Beim erstmaligen Nachweis der Nekrosen in der Bildgebung lag das mediane Alter der Patienten bei 16,2 Jahren.

Zu diesem Zeitpunkt waren 14 Patienten (93,3 \%) asymptomatisch. Eine Patientin klagte über Knochenschmerz in den Kniegelenken und wies entsprechend mehrere $\mathrm{ON}$ in den distalen Femora sowie in den proximalen Tibiae auf. Im Verlauf nahm die Zahl der Kinder und Jugendlichen mit Schmerzen zu. 8 Patienten gaben im Verlauf eine Symptomatik an (53,3 \%), 7 Patienten blieben schmerzfrei.

Die ausführliche Darstellung der betrachteten Patienten ist im Anhang (8.1) ersichtlich. 


\subsubsection{Vergleich der Verläufe der Patienten mit Morbus Hodgkin}

Insgesamt war zu sehen, dass sowohl ohne Therapie als auch unter Behandlung bezüglich der ON die Summe der Volumina aller aufgetretenen $\mathrm{ON}$ in einem Patienten im zeitlichen Verlauf überwiegend abnahm. Im Nachfolgenden sind die Tendenzen je nach Patientengruppen aufgeschlüsselt. Umso größer das initiale Gesamtvolumen der Nekrosen war, desto deutlicher war der häufiger auftretende Abfall des Graphen im Verlaufsdiagramm erkennbar. Neben der negativen Tendenz der Kurve überwogen auch stabile Verläufe im Gegensatz zu progressiven Entwicklungen der Summe der ON-Volumina.

Bei den Patienten ohne Behandlung der ON zeigte sich bei 5 der 7 Patienten eine Abnahme der Summe der ON-Volumina. Lediglich bei 2 Patienten war eine Zunahme des Gesamtvolumens aller ON zu verzeichnen. Bei Patient 6 war das initiale Gesamtvolumen sehr hoch und die betrachtete Verlaufsdauer sehr kurz, um die weitere Tendenz der ON-Volumina zu beurteilen. Die Summe der ON-Volumina bei Patient 7 nahm um $242 \%$ zu, was aber nur einem absoluten Wert von $6 \mathrm{ml}$ entsprach.

Der Verlauf der Patienten mit Therapie sollte in den Abschnitt vor der Therapie und in den Verlauf unter Therapie getrennt werden. Vor der Therapie nahm das Gesamtvolumen aller $\mathrm{ON}$ bei 7 von 8 Patienten ab. Dabei spiegelte sich die Tendenz, die bereits beim überwiegenden Teil der Kinder und Jugendlichen ohne jegliche Behandlung der ON gesehen wurde, wider. Bei Patient 10 konnte eine Zunahme der Summe der ON-Volumina im Vergleich zum initialen Wert verzeichnet werden. Das Gesamtvolumen zeigte allerdings in den ersten beiden Verlaufsbildgebungen, die vor Beginn der Therapie durchgeführt wurden, schon einen stabilen Wert. Unter der Therapie fand sich bei 7 der 8 Patienten eine Abnahme des Gesamtvolumens aller Nekrosen. Lediglich bei Patient 13 war eine Zunahme um $10 \%$ zu sehen, was einem absoluten Wert von nur $2 \mathrm{ml}$ entsprach. Es konnte keine Aussage getroffen werden, ob eine Therapie besser oder schlechter wirkte. Dafür waren erstens die Patientenzahlen zu gering und zweitens unterschied sich die Dauer der betrachteten Verläufe. 

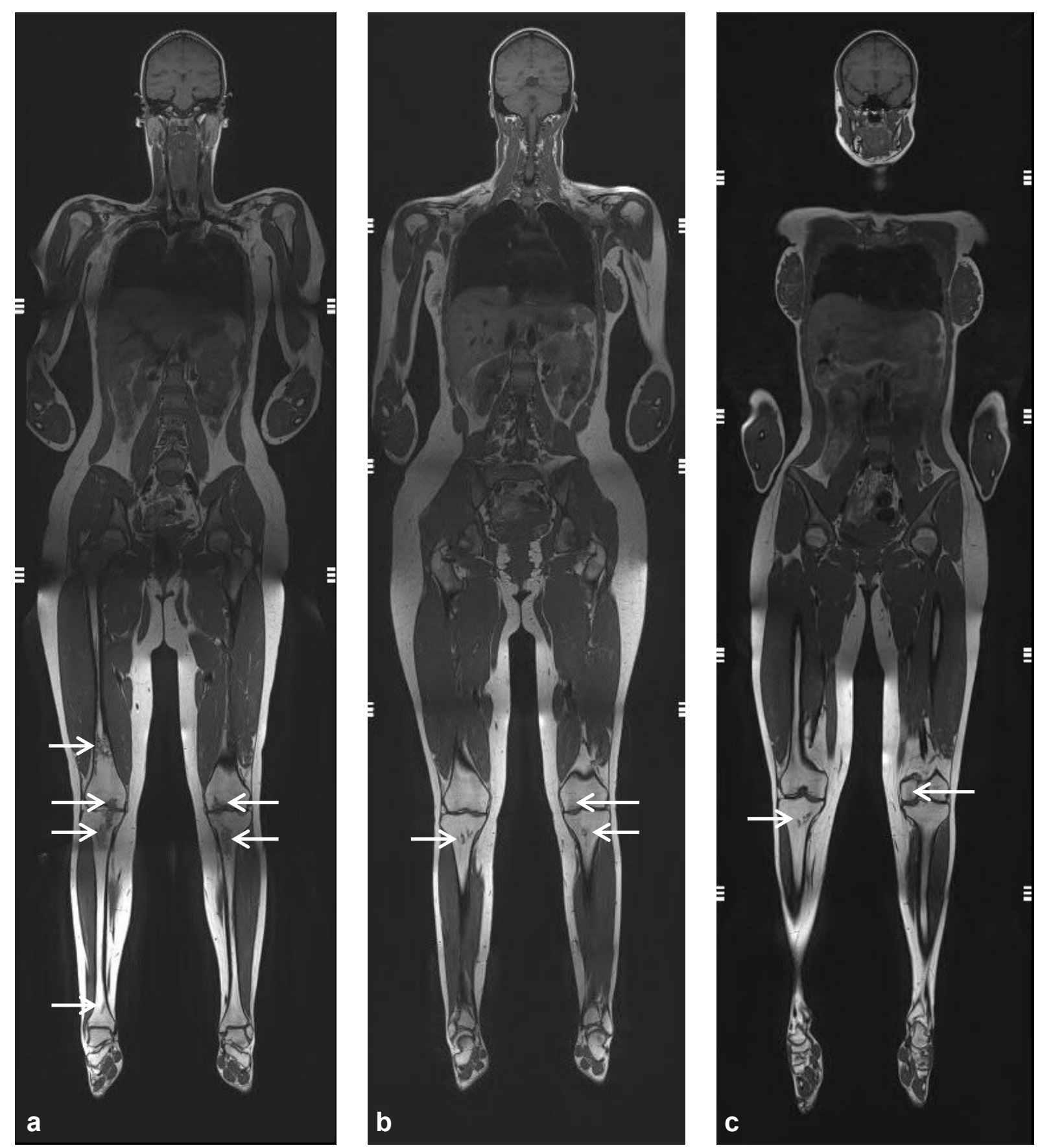

Abbildung 20: Zeitlicher Verlauf der Osteonekrosen $(\rightarrow)$ einer zur Diagnosestellung 15-jährigen Patientin ohne Therapie der Läsionen (Aufnahme a: Verdacht auf ON bei Schmerzen/erstes GK-MRT mit ON; b: 27 Monate danach; c: 52 Monate danach) 

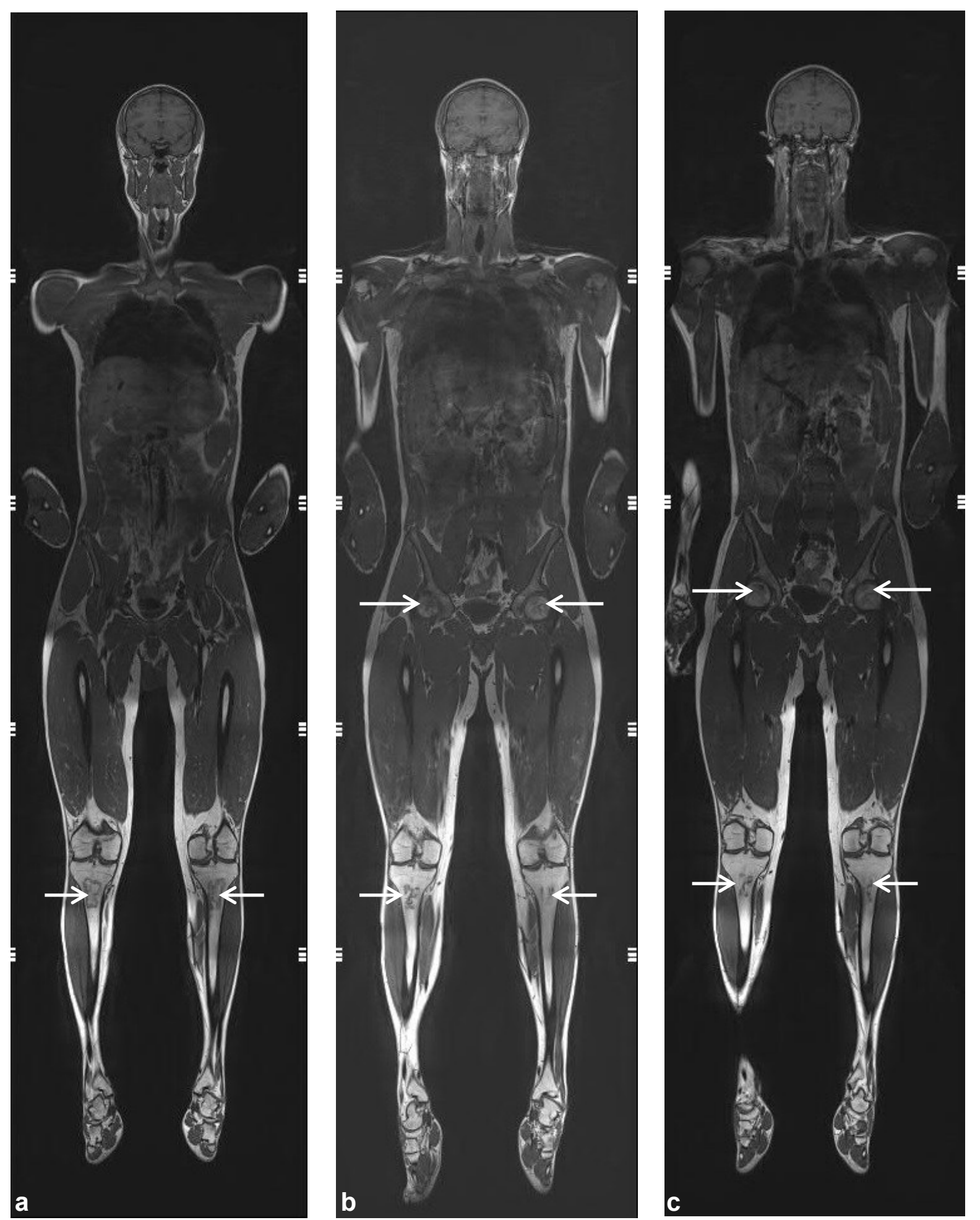

Abbildung 21: Zeitlicher Verlauf der Osteonekrosen $(\rightarrow)$ bei einem zur Diagnosestellung 17-jährigen Patienten mit Therapie der Läsionen (Aufnahme a: Diagnosestellung Morbus Hodgkin/erstes GK-MRT mit ON; b: 11 Monate danach unter Iloprosttherapie; c: 27 Monate danach (nach der Therapie)) 
Hinsichtlich der auftretenden Symptomatik war ein Unterschied zwischen den Patienten ohne und mit Therapie zu sehen, allerdings war die Anzahl der Patienten in den Gruppen zu klein, um aussagekräftige Ergebnisse zu zeigen. Zum Zeitpunkt der ersten GK-MRT mit Nachweis von ON war Patient 3 bereits symptomatisch. Dabei war zu beachten, dass bei dieser Patientin bereits zuvor in einem externen Klinikum ON in MRT-Aufnahmen gesehen wurden. Somit könnte bei dieser Patientin auch von einer Symptomatik im Verlauf gesprochen werden. 3 der 7 Patienten ohne Therapie waren bereits initial bzw. wurden im Verlauf symptomatisch (43\%). Unter den 8 Kindern und Jugendlichen, die aufgrund ihrer ON Behandlungen erhielten, wurden im Verlauf 5 Patienten symptomatisch (62,5\%). 


\section{Diskussion}

\subsection{Diskussion der Ergebnisse}

\subsubsection{Mögliche Sequenzen zur Darstellung von Osteonekrosen}

Die GK-MRT-Untersuchungen wurden an einem Siemens Avanto oder Siemens Symphony 1,5 Tesla-Gerät durchgeführt. Vier Sequenzen wurden auf ihre Eignung zur Darstellung von ON verglichen. Dabei handelte es sich um die T1-native SPACESequenz, die T2-native HASTE-Sequenz, die T2-HASTE-IR-Sequenz mit Fettunterdrückung mittels Inversion Recovery und die T1-SPACE-SPAIR-Sequenz mit Kontrastmittel und Fettsuppression. Wie in den Ergebnissen beschrieben, wurde der Wert „4" - entsprechend einer hochwertigen diagnostischen Qualität - für alle Sequenzen am häufigsten für die diagnostische Sicherheit als auch für den gesamten Qualitätsscore erreicht. Auch anhand der errechneten Mittelwerte zum Qualitätsscore sowie zu dem einzelnen Kriterium „diagnostische Sicherheit", was beschrieb, inwieweit die Läsionen als typisch für eine $\mathrm{ON}$ galten, konnte geschlussfolgert werden, dass die Darstellung der Nekrosen in allen vier Sequenzen gut bis sehr gut war. Die Mittelwerte für den Qualitätsscore betrugen für die T1-native Sequenz 3,8 $\pm 0,3$, für die T2-Wichtung $3,3 \pm 0,7$, für die T2-Sequenz mit Fettsuppression 3,6 $\pm 0,5$ und für die T1-Sequenz mit Kontrastmittel 3,8 $\pm 0,4$. Die entsprechenden Werte jeder Sequenz für die diagnostische Sicherheit lagen bei 3,6 \pm 0,7 für die T1-Wichtung, 2,7 \pm 1,2 für die T2-Sequenz, 3,1 \pm 0,9 für die T2Wichtung mit Fettsuppression und für die T1-Sequenz mit Kontrastmittel bei 3,6 \pm 0,7. Im Vergleich der Sequenzen waren hinsichtlich des Qualitätsscore sowie der diagnostischen Sicherheit die T1-native sowie die T1-Sequenz mit Kontrastmittel beiden T2-Wichtungen jeweils signifikant überlegen $(p<0,001)$. Im Vergleich der beiden T1-Sequenzen waren die Werte der nativen Sequenz in sehr wenigen Fällen größer als jene der T1-Wichtung mit Kontrastmittel. Der Unterschied war nicht statistisch signifikant ( $p=0,853$ bzw. $p=0,386$ ). Überwiegend fanden sich gleiche Werte für den Qualitätsscore bzw. die diagnostische Sicherheit dieser beiden Sequenzen. Die Werte für die beiden T2-Wichtungen konnten nicht im Signifikanztest verglichen werden, da pro Patient nur eine der beiden Sequenzen durchgeführt wurde. Allerdings war zu erkennen, dass der Qualitätsscore sowie der Wert für die diagnostische Sicherheit in der Gesamtheit höher bei der T2-HASTE-IR-Sequenz 
waren. Auch wurde der Wert „4“ häufiger in der Sequenz mit Fettsuppression erreicht als in der nativen T2-HASTE-Sequenz.

In der Literaturrecherche wurden bislang nur wenige Studien erfasst, die sich bereits mit der Detektion von ON mittels GK-MRT befassten. Miettunen et al. werteten an 11 pädiatrischen Patienten (5 männlich, 6 weiblich; medianes Alter bei Diagnose der ALL: 5,4 Jahre) mit muskuloskelettalen Schmerzen im Rahmen der ALL-Therapie koronare native T1-TSE- sowie koronare und axiale STIR-Aufnahmen hinsichtlich der Darstellung von ON aus (Miettunen et al. 2012). Die mittlere Untersuchungsdauer der gesamten Untersuchung lag bei den 9 Kindern, bei welchen ON nachgewiesen wurden, bei ca. 45 min. Die Autoren schlussfolgerten aus ihren Ergebnissen, dass die fettunterdrückte STIR-Sequenz die ideale Bildgebungsmethode zur Darstellung von $\mathrm{ON}$ sei und einen guten Kompromiss zwischen Untersuchungsdauer und Nachweis von Knochenläsionen darstelle. Dies sei vor allem für kleine Kinder und Schwerkranke vorteilhaft, da nur noch eine Sequenz durchgeführt werden müsste und sich somit die Sedierungs- bzw. Untersuchungszeiten verkürzen.

Beer et al. führten die GK-MRT-Aufnahmen an 5 Patienten (4 männlich, 1 weiblich; Alter: 13 - 16 Jahre) mit neu aufgetretenen Gelenk- oder Knochenschmerzen im Knie- oder Sprunggelenk nach einer Hochdosischemotherapie an einem Siemens Magnetom Symphony 1,5 Tesla-Gerät durch (Beer et al. 2008). Drei Patienten litten unter ALL, ein Patient hatte ein Medulloblastom und bei einem Patienten wurde eine chronisch myeloische Leukämie (CML) diagnostiziert. Bei 4 der 5 Patienten konnten ON in der GK-MRT gesehen werden. Zur Abdeckung des gesamten Körpers nutzten sie eine Kopf- sowie zwei Körperspulen. Die Dauer für die Aufnahme der wie in der vorliegenden Arbeit hauptsächlich vorkommenden 5 Körperregionen dauerte 60 - 90 Minuten. Die ersten vier Abschnitte wurden in koronarer und die Füße in sagittaler Schnittführung dargestellt. Die Autoren verwendeten ebenfalls das in der vorliegenden Arbeit genutzte Wertungsverfahren zur Beurteilung der qualitativen Darstellung der ON mit sechs verschiedenen Kriterien. Im Unterschied zu der eigenen Arbeit wurde anstatt der diagnostischen Sicherheit die Verdächtigkeit beurteilt. Die vergleichenden Sequenzen in der Studie von Beer et al. waren die fettunterdrückte T2-Turbo Inversion Recovery Magnitude (TIRM)-, die T1-TSE- und die fettgesättigte T1-TSE-Sequenz nach intravenöser Gabe von Gadolinium-haltigem Kontrastmittel. Die Mittelwerte des Qualitätsscore betrugen in der Analyse von Beer et al. 3,6 \pm 0,7 für die TIRM-Sequenz, 3,7 \pm 0,5 für die T1-Wichtung und 3,5 \pm 0,8 für 
die T1-Sequenz mit Kontrastmittel. Im Vergleich war keine der Sequenzen der jeweils anderen signifikant überlegen. Ein signifikantes Ergebnis erzielte der Vergleich der Mittelwerte für die Verdächtigkeit zwischen der TIRM- und der T1nativen Sequenz $(p=0,01)$. Die Werte für dieses Kriterium lagen für die TIRMSequenz bei 3,0 $\pm 0,8$, für die T1-Wichtung bei 3,6 $\pm 0,6$ und für die T1-Sequenz mit Kontrastmittel bei 3,1 $\pm 1,1$. Die beste Sichtbarkeit konnte mit der TIRM-Sequenz erreicht werden und die Sequenz mit den wenigsten Artefakten war die T1-native Wichtung. Im Vergleich zu der eigenen Studie war der Wert des Qualitätsscore in der T2-TIRM-Sequenz gering höher als in der T2-HASTE-Sequenz und gleich wie in der T2-HASTE-IR-Sequenz, aber niedriger als der Score der beiden T1-Wichtungen. Die Werte für die beiden Sequenzen in der T1-Wichtung waren in der Auswertung von Beer et al. etwas niedriger. Bezüglich der diagnostischen Sicherheit bzw. der Verdächtigkeit konnten in der nativen T1-Sequenz ähnliche Werte erreicht werden. Mit der T2-HASTE-IR-Sequenz wurden etwas höhere Werte und mit der T2-HASTESequenz gering niedrigere Werte für dieses Kriterium erreicht als in der T2-TIRMSequenz. Für die T1-Sequenz mit Kontrastmittel lag der Wert in der Studie von Beer et al. niedriger.

Zusammenfassend kann gesagt werden, dass alle Sequenzen sowohl in der Studie von Beer et al. als auch in der vorliegenden Arbeit ON gut darstellten. Der Grund für die höheren Werte für die T2-TIRM- sowie T2-HASTE-IR-Sequenz gegenüber der reinen T2-HASTE-Sequenz könnte darin bestehen, dass bei den beiden erstgenannten Sequenzen immer eine Fettsuppression vorlag, sodass ein höherer Kontrast zwischen ON und gesundem Knochen bestand. Aus diesem Grund wurden bei diesen beiden Sequenzen in den zwei unterschiedlichen Studien annähernd gleiche Ergebnisse erreicht. Die niedrigeren Werte in den beiden T1-Wichtungen in der Studie von Beer et al. könnten einerseits in der verwendeten Repetitionszeit TR und andererseits im betrachteten Patientengut begründet sein. Beide nativen T1Sequenzen waren TSE-Aufnahmen und bei beiden T1-Sequenzen mit Kontrastmittel wurde eine Fettsättigung verwendet. Für die T1-Sequenzen in der vorliegenden Arbeit wurde eine TR von $430 \mathrm{~ms}$ verwendet, bei Beer et al. lag diese bei $690 \mathrm{~ms}$. Je kürzer die TR ist, umso stärker wird der Bildkontrast in der T1-Sequenz beeinflusst. Die Echozeiten TE mit $11 \mathrm{~ms}$ in der vorliegenden Arbeit und $12 \mathrm{~ms}$ bei Beer et al. unterschieden sich hingegen nur sehr gering. Durch die kurze TE wurde der T2Kontrast vermindert. Da in der Studie von Beer et al. nur 4 Patienten mit ON 
betrachtet wurden, ist eine vergleichende Aussage zwischen dem Patientengut in den beiden Studien nicht ausreichend möglich. Bei den Patienten mit Leukämie wird das Knochenmarksignal durch die Blasteninfiltration stark verändert, wodurch die ON maskiert werden könnten. Bei $75 \%$ der Patienten mit ON bei Beer et al. und 32,6 \% der Patienten mit ON in der vorliegenden Arbeit wurde eine Leukämie diagnostiziert. Es könnte somit davon ausgegangen werden, dass die gering qualitativ schlechtere Darstellung der ON in den T1-Sequenzen der Studie von Beer et al. durch den höheren Anteil der Patienten mit Leukämie sowie durch die längere Repetitionszeit begründet war.

Weitere Studien bezogen sich auf die Darstellung der ON mit Hilfe der MRT einzelner Skelettregionen. Engelbrecht et al. bestätigten einen zuverlässigen Nachweis der ON mit Hilfe der T1-Sequenz aufgrund dem typischen Bild der Läsionen mit einem hypointensen Rand (Engelbrecht et al. 2000). Laut Saini und Saifuddin wurde eine deutlichen Darstellung der Nekrosen mittels einer T1-SpinEcho- und einer fettsupprimierten T2-TSE- oder STIR-Sequenz erreicht (Saini und Saifuddin 2004). Ippolito et al. führten MRT-Untersuchungen der unteren Extremität an Philips Achieva 1,5 Tesla-Geräten sowie an Philips Panorama 1,0 Tesla-Geräten durch (Ippolito et al. 2016). Vor allem zur Detektion und Charakterisierung von frühen ON zeigte sich bei der MRT eine hohe Sensitivität sowie Spezifität für die T1- sowie die STIR-Sequenz in koronarer Schnittführung. Die koronare Ebene war laut den Autoren besonders bei der Beurteilung der Progression zur Gelenkdeformität vorteilhaft. Von Stillfried und Weber schlugen zum Nachweis vom Knochenmarködem als Frühstadium einer ON eine fettunterdrückte T2-Sequenz vor (von Stillfried und Weber 2014). Solch eine fettsupprimierende Sequenz, wie es die STIR-Sequenz ist, wurde auch von Kubo et al. zusätzlich zur T1-Wichtung zur Darstellung von $\mathrm{ON}$ des Femurkopfs bei einem 45-jährigen Patienten mit hämophagozytischem Lymphozytose-Syndrom genutzt (Kubo et al. 2015). Mit Hilfe eines Philips Achieva 1,5 Tesla-Gerät wurden MRT-Aufnahmen in koronarer sowie axialer Schnittführung durchgeführt. Frühe Läsionen, d.h. schon drei Wochen nach Beginn der systemischen Steroidtherapie, konnten hyperintens in der STIR-Sequenz gesehen werden. An diesen Skelettlokalisationen wurden nach 3 Monaten Nekrosen mit hypointensem Rand in der T1-Wichtung detektiert. Die Darstellung in der T1nativen Sequenz galt als diagnostisches Kriterium für eine ON. Der Nutzen der schwach dargestellten sehr frühen signalreichen Läsion in der STIR-Sequenz sollte 
mit Vorsicht betrachtet werden. Denn ein Problem der frühen Entdeckung und somit meist auch Behandlung von ON liegt in der deutlich steigenden Inzidenz von transienten Knochenmarködemen als mögliche Vorstufen der Entwicklung einer ON (Gaynon 2015). Mit Hilfe der MRT kann dieses reversible Stadium nicht vom Nekrosestadium I der ARCO-Klassifikation unterschieden werden. Die Verlaufsbeobachtung ist in dieser Situation erforderlich, um sich entwickelnde ON sicher zu detektieren (von Stillfried und Weber 2014).

Aus den Vergleichen zwischen den Sequenzen bei der vorliegenden Arbeit konnte geschlossen werden, dass entweder die T1-native Sequenz oder die T1-Sequenz mit Kontrastmittel genutzt werden sollte, um ON in der GK-MRT sicher nachzuweisen. Aufgrund der minimalen Überlegenheit der T1-nativen Wichtung gegenüber der T1SPACE-SPAIR-Sequenz sowie der zusätzlichen Belastung durch das Kontrastmittel, ist die Durchführung der T1-nativen Sequenz empfehlenswert. Gadolinium-DTPAKomplexe als Kontrastmittel verbleiben zum Teil im Körper und lagern sich vor allem im Knochen, aber auch in der Haut, der Leber und im Gehirn ab. Vor allem die Ablagerung im Gehirn wird aktuell hinsichtlich möglicher pathologischer Auswirkungen erforscht und ist oft Teil einer Diskussion zwischen den Eltern der pädiatrischen Patienten und dem aufklärenden Radiologen. Kontrastmittel sollte deshalb möglichst eingespart werden (Klee et al. 2017). In der vorliegenden Arbeit wurde bei $72,5 \%$ der $\mathrm{ON}$ in der T1-nativen Wichtung der Wert 4 für die diagnostische Sicherheit erreicht. Somit konnte in einem großen Teil der Fälle auch ohne Kontrastmittel eine Festlegung getroffen werden, ob es sich sicher um eine ON handelte.

Zusätzlich zu der T1-nativen Sequenz sollte die T2-Wichtung mit Fettsuppression in die Untersuchung eingeschlossen werden, um mögliche Begleitbefunde in den Gelenken sowie Weichteilen nicht zu übersehen. Laut der Studie von Beer et al. waren die T2-TIRM- bzw. die T1-Sequenz mit Kontrastmittel hilfreich bei der Beurteilung der mitbeteiligten Weichteile und somit für die Verlaufskontrollen wichtig, bei denen nicht nur die ON zu beurteilen sind (z.B. Staging, Restaging, Fokussuche) (Beer et al. 2008). Für die Autoren reichten zum Nachweis der ON die TIRMSequenz aufgrund der besten Sichtbarkeit der Läsionen sowie die T1-TSE-Sequenz mit der höchsten Gesamtbildqualität und vor allem der besten Verdächtigkeit bezüglich einer Nekrose bei wenigen Artefakten aus. 


\subsubsection{Häufigkeit der Osteonekrosen und Symptomatik}

In dieser retrospektiven Studie wurden bei 41,0 \% der 105 eingeschlossenen Patienten ON in der GK-MRT diagnostiziert. Dieser Anteil spiegelte sich auch in den beiden Patientengruppen wider. Für die 63 Patienten ohne SZT (36 männlich, 27 weiblich; mittleres Alter bei Diagnose der Grunderkrankung: 11,1 \pm 5,6 Jahre, Median: 12,9 Jahre) lag dieser bei 41,3\% und unter den 42 Jungen und Mädchen, die sich einer SZT unterziehen mussten (26 männlich, 16 weiblich; mittleres Alter bei der ersten SZT: 10,7 \pm 6,5 Jahre, Median: 12,0 Jahre), bei 40,5\%. Dabei wurden sowohl symptomatische als auch asymptomatische Patienten mit der GK-MRT untersucht.

Eine ähnliche Inzidenz von 41,7 \% ermittelten auch Littooij et al. in ihrer prospektiven Studie, in welcher 24 Kinder und Jugendliche (12 männlich, 12 weiblich; mittleres Alter 15,1 Jahre) mit der Diagnose Morbus Hodgkin eingeschlossen wurden (Littooij et al. 2017). GK-MRT-Untersuchungen wurden initial, nach zwei Zyklen der Chemotherapie sowie bei 16 Patienten zusätzlich nach Ende der Chemotherapie durchgeführt. Bei 10 Patienten konnten $\mathrm{ON}$ nachgewiesen werden. Im Unterschied zu der vorliegenden Arbeit wurde hier eine homogene Gruppe von Patienten, die alle an Morbus Hodgkin litten, eingeschlossen.

Die höchste Inzidenz an ON mit $73,9 \%$ berichteten Ippolito et al. in ihrer retrospektiven Studie, bei welcher 73 Kinder mit ALL (37 männlich, 36 weiblich; mittleres Alter 12,4 Jahre) bezüglich des Auftretens von ON in der unteren Extremität mit Hilfe der MRT begutachtet wurden (Ippolito et al. 2016). 45 Patienten erhielten eine Chemotherapie und 28 Patienten zusätzlich eine SZT. Es wurden wie in der hier vorliegenden eigenen Studie bezüglich der ON sowohl symptomatische als auch asymptomatische Kinder eingeschlossen. Auch Kawedia et al. ermittelten in ihrer prospektiven Analyse von 364 Kindern (198 männlich, 166 weiblich) mit ALL eine hohe Inzidenz für das ON-Auftreten von 71,8 \% (Kawedia et al. 2011). Dabei wurden MRT-Aufnahmen der Hüfte sowie der Kniegelenke von Patienten mit Schmerzen als auch von Patienten ohne Symptomatik durchgeführt. Die Häufigkeit der Patienten mit ON war in den beiden zuletzt genannten Studien deutlich größer als in der vorliegenden Studie. Es könnte darin begründet sein, dass nur ALL-Patienten begutachtet wurden, bei denen ON häufiger auftreten, während in den eigenen Daten eine Vielzahl verschiedener onkologischer Erkrankungen analysiert wurde. Ebenfalls spielt die betrachtete Verlaufszeit eine Rolle. Bei der eigenen Studie wurde 
unabhängig von der Zeit zwischen Diagnosestellung der Grunderkrankung bzw. der ersten SZT bis zum Auftreten der ON der erstmalige Nachweis der Nekrosen in der GK-MRT erfasst.

Hinsichtlich der Häufigkeit der ON-Manifestation nach einer SZT fanden Sharma et al. eine Prävalenz von 29,5 \% innerhalb von 3 Jahren nach einer allogenen SZT (Sharma et al. 2012). Es wurden in diese retrospektive Analyse 149 Kinder (84 männlich, 65 weiblich; medianes Alter 11 Jahre) mit und ohne Schmerzsymptomatik eingeschlossen. MRT-Aufnahmen wurden von der Hüfte oder dem Kniegelenk durchgeführt. Im Vergleich zur eigenen Studie fand sich hier eine ähnliche Häufigkeit von ON. Es wurden in beiden Studien Patienten mit unterschiedlichen Grunderkrankungen mit Hilfe der MRT nach einer SZT untersucht. Alle drei letztgenannten vergleichenden Studien führten lediglich MRT-Untersuchungen der unteren Extremität durch.

Miettunen et al. führten eine bereits angesprochene retrospektive Analyse an 32 pädiatrischen Patienten mit ALL durch, wovon 11 Kinder (5 männlich, 6 weiblich; medianes Alter bei Diagnose der ALL: 5,4 Jahre) persistierende muskuloskelettale Schmerzen während der Leukämietherapie beklagten (Miettunen et al. 2012). Bei diesen Patienten wurde daraufhin eine GK-MRT-Untersuchung durchgeführt. 9 Patienten wiesen ON auf, entsprechend $28 \%$ aller eingeschlossenen Patienten mit ALL. Die Häufigkeit war in dieser Analyse geringer als in der eigenen Studie. Dies lag darin begründet, dass Miettunen et al. nur symptomatische Patienten untersuchten. Insgesamt gesehen waren die Häufigkeiten des Auftretens von ON in der Bildgebung nur schwer zu vergleichen. In vielen Studien wurden nur Patienten mit Schmerzen eingeschlossen und die Betrachtung geschah teils nur an einem Zeitpunkt, teils über einen sehr langen Zeitraum. Auch die Definitionen der ON waren unterschiedlich. Nicht zuletzt spielten die eingeschlossenen Patienten eine Rolle, ob es sich dabei um eine homogene Gruppe handelte oder diese unterschiedliche Grunderkrankungen aufwiesen mit verschiedenen Therapieprotokollen.

Die Dauer zwischen der Diagnose der Grunderkrankung bei den Patienten ohne SZT bis zum erstmaligen Auftreten von $\mathrm{ON}$ in der GK-MRT lag im Median bei 6,0 Monaten (Minimum 0,0 Monate; Maximum 80,5 Monate). Die minimalen Werte von 0,0 Monate bei einer Hodgkin-Patientin und 0,4 Monate bei einem Patienten mit NHL zeigten, dass die ON bei diesen Patienten bereits zum Zeitpunkt der 
Diagnosestellung der Grunderkrankung sowie vor Beginn der Chemotherapie bestanden. Niinimäki et al. konnten in ihrer Analyse von 32 Kindern (20 männlich, 12 weiblich; mittleres Alter 7,1 Jahre) mit unterschiedlichen Grunderkrankungen bei 6 Patienten ON nachweisen (Niinimaki et al. 2008). Bei allen von Nekrosen betroffenen Kindern bestanden die Diagnosen NHL oder Morbus Hodgkin. Somit kamen die Autoren zu der Schlussfolgerung, dass Lymphome an sich als Ursache für die Entwicklung von ON gelten könnten, da sie das Knochenmark möglicherweise involvieren. Dies könnte auch die Erklärung für das Auftreten von ON in der GK-MRT noch vor Beginn der Chemotherapie bei den beiden Patienten in der vorliegenden Studie sein.

Der Ausreißer von 80,5 Monaten zwischen der Diagnose der Grunderkrankung und der erstmaligen Detektion von ON in einer GK-MRT fand sich bei einer Patientin mit ALL. In den vorhandenen Akten war allerdings dokumentiert, dass die ON dieser Patientin bereits in einer früheren MRT-Untersuchung der Hüftregion festgestellt wurden. Die Dauer zwischen der ALL-Diagnose und dieser MRT-Aufnahme lag bei nur 14 Monaten. Dies wurde allerdings in der vorliegenden Studie nicht gewürdigt, da nur die Diagnose der ON in einer Ganzkörper-Aufnahme eingeschlossen wurde.

Eine ähnlich wie in der eigenen Studie mediane Dauer wurde auch in der schon erwähnten GK-MRT-Analyse bei Kindern mit ALL von Miettunen et al. mit 4,9 Monaten ermittelt (Miettunen et al. 2012). Dies war das geringste Zeitintervall zwischen Diagnose einer ALL und Auftreten von ON, das in der Literatur beschrieben wurde. Die maximale Dauer zwischen Diagnosestellung einer akuten Leukämie und dem radiologischen Nachweis von ON mittels MRT betrug in der Studie von Girard et al. im Median 22 Monate (Girard et al. 2013).

Bei den Patienten mit SZT lag die Dauer zwischen der ersten Transplantation und dem ersten Nachweis von ON in der GK-MRT im Median bei 9,0 Monaten (Minimum 0,8 Monate; Maximum 223,7 Monate). Bei dem Ausreißer von 223,7 Monaten handelte es sich um einen Patienten mit Morbus Hodgkin, welcher nach seiner SZT noch mehrere Rezidive seiner Grunderkrankung erlitten hatte. Ob die SZT oder die Rezidive und ihre Behandlung als Ursache für die ON-Entstehung gelten können, konnte somit nicht definiert werden. Ein ähnliches medianes Intervall von 0,9 Jahren zwischen der ersten SZT und dem Auftreten von symptomatischen ON beschrieben Girard et al. in ihrer Studie (Girard et al. 2013). Sie untersuchten insgesamt 943 Kinder mit Leukämie, wovon sich 256 Patienten (162 männlich, 94 weiblich; mittleres 
Alter bei der SZT: 8,65 \pm 0,3 Jahre) einer SZT unterziehen mussten. Von denen entwickelten 15 Kinder (5,9 \%) ON, welche mit Hilfe einer MRT-Untersuchung des symptomatischen Gelenks nachgewiesen wurden. Der zeitliche Abstand zwischen Transplantation und Detektion von ON von im Median 0,9 Jahren wurde auch von Faraci et al. angegeben und entsprach dem kürzesten Intervall, das in der Literatur zu finden war (Faraci et al. 2006). Die maximale Dauer zwischen SZT und erster Detektion von ON wurde in der Studie von Campbell et al. mit im Mittel von 38 Monaten beschrieben (Campbell et al. 2009).

Die Angabe zu der Dauer bis zum ON-Auftreten in der GK-MRT war stark davon abhängig, nach welcher Indikation (Routinekontrolle oder Klinik) und in welchen Abständen die Bildgebung durchgeführt wurde. In der vorliegenden Studie war dies sehr unterschiedlich. Weder das erste noch die darauffolgenden GK-MRTAufnahmen lagen in einem einheitlichen Abstand zu der Diagnosestellung der Grunderkrankung bzw. zu der ersten SZT. Um ein annähernd aussagekräftiges Ergebnis zu erhalten, wurde deshalb auch der Median zur Auswertung ermittelt.

Zum Zeitpunkt des erstmaligen Nachweises von ON in der GK-MRT waren 60,5 \% der Patienten in der vorliegenden Studie asymptomatisch, 39,5 \% der Kinder und Jugendlichen beklagten Knochenschmerzen vorwiegend in der unteren Extremität. Daran war erkennbar, dass der größte Teil der Patienten, bei denen sich ON entwickelten, keine klinischen Anzeichen vorwiesen. Bei den 15 Patienten mit Morbus Hodgkin, welche im Verlauf betrachtet wurden, war initial nur eine Patientin symptomatisch. Bei dieser Patientin waren allerdings bereits zuvor in einem externen Klinikum ON in MRT-Aufnahmen detektiert worden. Im Verlauf stieg der Anteil an symptomatischen Patienten auf 53,3 \%. Alle 8 Patienten beklagten Schmerzen in der unteren Extremität.

Auch in der bereits erwähnten retrospektiven Studie von Beer et al. konnten die symptomatischen $\mathrm{ON}$ bei den 4 Patienten mit Nachweis von $\mathrm{ON}$ in den Beinen gefunden werden (Beer et al. 2008). Zusätzlich wurden bei diesen Patienten 19 weitere asymptomatische Läsionen mit einem Anteil von $79 \%$ an den insgesamt 24 ON nachgewiesen. Diese befanden sich auch in der oberen Extremität. Somit war zu schlussfolgern, dass symptomatische Nekrosen überwiegend in den Beinen zu finden waren. Dagegen bereiteten Läsionen der oberen Extremität vorwiegend keine Schmerzen. In den Beobachtungen der vorliegenden Arbeit sowie in der 
angesprochenen GK-MRT-Studie von Miettunen et al. wurde dies auch bestätigt (Miettunen et al. 2012). In der Analyse von Miettunen et al. beklagten $100 \%$ der Patienten mit ON in der unteren Extremität Beinschmerzen, wohingegen nur $33 \%$ der Kinder mit ON der oberen Extremität Beschwerden in den Armen zeigten. Die vor allem in den Beinen auftretende Symptomatik ist darin begründet, dass diese die gewichtstragenden und somit stärkeren Belastungen ausgesetzten Extremitäten sind.

Karimova et al. zeigten ebenfalls eine positive Korrelation zwischen der Lokalisation der ON in der MRT und der beobachteten Symptomatik (Karimova et al. 2006). Symptomatische ON wurden in der Studie von Karimova et al. vor allem in den Metaund Diaphysen der Tibia sowie in den Epiphysen des Femurs beobachtet. Im Oberschenkelknochen war die Korrelation zwischen ON und Symptomatik vor allem bei zunehmender Größe und Gelenkflächenbeteiligung der Nekrosen signifikant ( $p<$ 0,001). Asymptomatische $\mathrm{ON}$ wiesen deutlich seltener eine Gelenkflächenbeteiligung auf als symptomatische ON. Je größer der Umfang der einbezogenen Gelenkfläche war, desto stärker war die Symptomatik.

Der große Anteil der asymptomatischen ON sollte im weiteren Krankheitsverlauf allerdings nicht unberücksichtigt bleiben, da die $\mathrm{ON}$ an Größe zunehmen und sich in die gelenknahen Regionen ausdehnen können. Deshalb ist es von Vorteil, diese sowie die symptomatischen Läsionen mit Hilfe der GK-MRT-Untersuchung in regelmäßigen Intervallen zu untersuchen, bevor der Anteil der ON mit Schmerzen ansteigt und Knocheneinbrüche bzw. vorzeitiger Gelenkverschleiß auftreten. Die im Verlauf zunehmende Symptomatik konnte durch Ergebnisse der prospektiven Studie von Flouzat-Lachaniette et al. bestätigt werden (Flouzat-Lachaniette et al. 2016). Sie untersuchten 200 erwachsene Patienten (124 männlich, 76 weiblich; mittleres Alter 39 Jahre), bei denen ON im Rahmen der Steroidtherapie auftraten. Der Verlauf dieser ON unter weiterer Steroidgabe wurde mit Hilfe von MRT-Aufnahmen der betroffenen Gelenke analysiert. Die Symptomatik nahm vor allem im Bereich der Hüfte von $58 \%$ auf $100 \%$ bei den Patienten mit ON in dieser Region zu. Ein Progress der symptomatischen Läsionen fand sich auch bei $\mathrm{ON}$ in den Knie- sowie Sprunggelenken. Auch im Schulterbereich wuchs der Anteil der ON mit Schmerzsymptomatik von initial $24 \%$ auf $70 \%$.

Innerhalb von drei Jahren nach Beginn der ALL-Chemotherapie werden laut Mattano et al. fast alle Patienten symptomatisch (Mattano et al. 2000). Diese Aussage konnte 
auch in der eigenen Studie bei den wenigen Patienten mit Morbus Hodgkin, die über einen Zeitraum von drei Jahren beobachtet wurden, bestätigt werden.

\subsubsection{Lokalisationen der Osteonekrosen}

In vielen Studien zur Beurteilung von ON wurden aufgrund des häufigeren Auftretens lediglich MRT-Untersuchungen der unteren Extremität durchgeführt. Um generelle, aussagekräftigere Ergebnisse zu den am häufigsten betroffenen Knochen und gelenknahen Knochenregionen zu erhalten, sollte allerdings das gesamte Skelett mittels GK-MRT abgebildet werden. Neben den ON sind in der GK-MRT die Weichteile und inneren Organe mit zu beurteilen, was insbesondere bei onkologischen Patienten vorteilhaft ist. Wie bereits erwähnt konnten zur Bedeutung der GK-MRT im ON-Nachweis bislang nur wenige Studien in der Literatur gefunden werden.

Beer et al. schlossen in ihre retrospektiven Analyse fünf Patienten ein, die im Rahmen einer Hochdosischemotherapie über Knochenschmerzen klagten (Beer et al. 2008). 19 der insgesamt 24 beobachteten ON wurden in der unteren Extremität (Femur, Knochen um das Kniegelenk, Tibia, Knochen um das Sprunggelenk) und 5 Läsionen im Humerus diagnostiziert. Eine weitere retrospektive Analyse führten Miettunen et al. durch (Miettunen et al. 2012). Bei 9 Patienten mit einem positiven Nachweis von ON in der GK-MRT konnten insgesamt 66 Läsionen in 42 Epiphysen der langen Röhrenknochen und 63 zusätzliche Nekrosen an anderen Lokalisationen gefunden werden. Dies spiegelte bereits wider, dass vor allem die Epiphysen von ON betroffen waren, in dieser Studie am meisten jene des Femurs. Bei $57 \%$ der epiphysären Nekrosen in den Knochen der Knieregion sowie bei 46 \% der Läsionen in der distalen Tibia fanden die Autoren eine Gelenkfächenbeteiligung von > $50 \%$. Die 63 weiteren, nicht-epiphysären Läsionen befanden sich am häufigsten in diametaphysären Regionen jener Knochen, wo auch die Epiphyse betroffen war. Von diesen Nekrosen waren $78 \%$ in den Beinen und $22 \%$ in der oberen Extremität zu finden. Alle 9 Patienten wiesen multifokale ON mit vorwiegender Lokalisation in den langen Röhrenknochen Femur, Tibia und Humerus auf.

Eine prospektive GK-MRT-Studie zur Detektion von ON bei 24 Kindern und Jugendlichen mit Morbus Hodgkin führten Littooij et al. durch (Littooij et al. 2017). Bei 8 der 10 Patienten mit ON wurden die Läsionen in den langen Röhrenknochen gefunden, dabei am häufigsten im distalen Femur und der proximalen Tibia. 24 der insgesamt 38 gefunden ON in den langen Röhrenknochen $(63,2 \%)$ waren in der 
unteren Extremität lokalisiert. Albano et al. werteten ebenfalls GK-MRTUntersuchungen von 42 Patienten (20 männlich, 22 weiblich; mittleres Alter 29,2 Jahre) mit Morbus Hodgkin aus und fanden bei 7 Patienten (17\%) insgesamt 48 ON (Albano et al. 2017). Diese waren zu 73 \% in der unteren Extremität lokalisiert. 48 \% aller ON wurden im Bereich der Kniegelenke nachgewiesen. Bei 6 von 7 Patienten mit ON (86 \%) traten die Läsionen multifokal auf. Als Limitation dieser GK-MRTStudien war die geringe Anzahl an Patienten mit ON zu vermerken.

In der vorliegenden Arbeit konnten ähnliche Ergebnisse bezüglich der Lokalisation der ON im Skelett pädiatrischer Patienten gefunden werden. Insgesamt wurden 546 verschiedene Nekrosen bei 43 Patienten mit ON, davon 268 Läsionen bei den Patienten ohne SZT und 278 Nekrosen bei den Kindern und Jugendlichen mit SZT, in den GK-MRT-Aufnahmen detektiert. Im Median waren 9,0 ON pro Patient nachzuweisen, woraus geschlossen werden konnte, dass die Läsionen zum größten Teil multifokal im Skelett auftraten. Aus der sehr oft gesehenen Multifokalität schlossen Flouzat-Lachaniette et al., dass die ON alle zur gleichen Zeit bzw. in kurzen Zeitintervallen entstanden sein müssten (Flouzat-Lachaniette et al. 2016).

Unabhängig davon, ob in der vorliegenden Studie das gesamte Patientenkollektiv oder die einzelnen Gruppen ohne bzw. mit SZT analysiert wurden, war durchgehend die untere Extremität zu > $80 \%$ unter allen betrachteten ON betroffen. Vor allem bei den Kindern und Jugendlichen ohne SZT konnte mit 94,4 \% ein deutliches Ergebnis gezeigt werden. Der Grund lag darin, dass der mechanische Stress auf die Knochen der gewichtstragenden unteren Extremität höher ist.

Wie in den beschriebenen Studien von Littooij et al. und Albano et al. konnten auch in der vorliegenden Arbeit $\mathrm{ON}$ in den Knochen der Knieregion am häufigsten gesehen werden. 26,1 \% der ON aller Patienten sowie 30,5\% der Läsionen bei den Kindern und Jugendlichen ohne SZT und 21,5 \% der Nekrosen bei den Jungen und Mädchen mit SZT waren in den kniegelenknahen Knochenregionen zu finden. Aus diesem Grund wurden die Läsionen in diesen Knochenbereichen genauer analysiert. 60,2 \% der Nekrosen fanden sich im distalen Femur, dort vor allem epiphysär aber auch metaphysär. In der proximalen Tibia lagen die ON überwiegend metaphysär. Wie in der erwähnten Studie von Miettunen et al. konnten in der vorliegenden Arbeit die meisten $\mathrm{ON}$ in den Epiphysen (36,9 \%) gefolgt von den Läsionen in den Metaphysen $(24,7$ \%) der langen Röhrenknochen detektiert werden. Diese Tendenz konnte wie bereits beschrieben auch bei der getrennten Betrachtung der ON in den 
kniegelenknahen Knochenbereichen gesehen werden. Der Grund für das häufigere Auftreten in den Epi- und Metaphysen der Knochen lag in der sich in diesem Areal befindenden Wachstumsfuge. In diesem Bereich sind das arterielle Angebot sowie die venöse Drainage limitiert (Scherer et al. 2001). Mit der Knochenreifung und dem Längenwachstum der Knochen während der Pubertät steigt der Verbrauch an Metaboliten und Sauerstoff an. Entlang der Epiphysenfuge kann es zu Hypoxie kommen, da das Angebot des Blutflusses nicht mehr dem Bedarf entspricht. Durch den Wachstumsfugenschluss verliert der Knochen die Möglichkeit der Pufferung des erhöhten mechanischen Stresses und somit die Fähigkeit, dem erhöhten intramedullären Druck standzuhalten (Kunstreich et al. 2016).

Auch sollte wie bereits in der Studie von Miettunen et al. beschrieben die Gelenkflächenbeteiligung jener $\mathrm{ON}$, welche die Epiphyse erreichen, in der Bildgebung beurteilt werden. In der vorliegenden Arbeit konnte unter den 199 betrachteten Läsionen bei 54,3 \% eine Beteiligung der Gelenkfläche gesehen werden. Karimova et al. analysierten in ihrer retrospektiven Studie, bei der 109 Kinder und Jugendliche ( 57 männlich, 52 weiblich; medianes Alter bei der Diagnose: 11,5 Jahre) mit hamätologischen, malignen Erkrankungen eingeschlossen wurden, mit Hilfe der MRT das Auftreten von ON in kniegelenknahen Knochen (Karimova et al. 2010). Bei einer Gelenkflächenbeteiligung der Nekrosen erhöhte sich das Risiko für einen Gelenkeinbruch. Gelenkeinbrüche wurden von Karimova et al. vor allem bei Läsionen, die mehr als 50 \% der Gelenkfläche einnahmen, beobachtet.

Wie in der vorliegenden Studie fanden auch Ippolito et al. bei der Betrachtung von ON in der unteren Extremität keine bevorzugte Lokalisation bezüglich der rechten oder linken Körperseite (Ippolito et al. 2016).

\subsubsection{Einflussfaktor Alter auf das Auftreten von Osteonekrosen}

In der vorliegenden Studie konnte eine Korrelation zwischen höherem Patientenalter und einem Risiko für das Auftreten von ON in der GK-MRT ermittelt werden. Unter den Patienten, die sich keiner SZT unterziehen mussten, konnten bei 7,4 \% der 27 Patienten mit einem Alter von $\leq 10$ Jahren bei der Diagnosestellung der Grunderkrankung $\mathrm{ON}$ in der Bildgebung nachgewiesen werden. Unter den 36 Patienten ohne SZT mit einem Alter von > 10 Jahren waren die Läsionen bei 66,7 \% zu sehen. Die älteren Patienten hatten ein signifikant 9,6-mal höheres Risiko für ein Auftreten von ON in der GK-MRT $(p=0,002)$. Auch bei den Patienten mit SZT war die Häufigkeit des Nachweises von ON in den GK-MRT-Aufnahmen bei den 
Patienten mit einem Alter von > 10 Jahren bei der ersten SZT höher. 50,0 \% der 24 älteren Patienten wiesen Nekrosen in der GK-MRT vor. Unter den 18 Kindern mit einem Alter von $\leq 10$ Jahren fanden sich fünf Patienten mit $\mathrm{ON}$ in der Bildgebung. Das Risiko für die älteren Patienten war hier 1,8-mal höher im Vergleich zu den Kindern mit einem Alter von $\leq 10$ Jahren $(p=0,293)$.

Auch in vielen anderen Studien konnte das erhöhte Risiko bei Kindern mit einem Alter von über 10 Jahren für die Entwicklung von ON belegt werden. Girard et al. untersuchten in ihrer Analyse 943 Kinder (520 männlich, 423 weiblich; mittleres Alter bei Diagnose: 6,35 \pm 0,14 Jahre) mit Leukämie, wovon bei 24 Patienten $O N$ in der MRT des symptomatischen Gelenks auftraten (Girard et al. 2013). Dabei hatten 9 Patienten nur eine Chemotherapie und 15 Kinder zusätzlich eine SZT erhalten. In beiden Patientengruppen konnte ein signifikant höheres Risiko für die Entwicklung von ON bei den Kindern mit einem Alter von > 10 Jahren bei der Diagnose bzw. bei der SZT gefunden werden ( $p<0,001)$. Trotz der vielen eingeschlossenen Patienten hatte diese Studie nur wenige Kinder, bei denen ON auftraten.

In der prospektiven Studie von Kawedia et al. mit 364 Kindern mit ALL wurde ebenfalls das Alter als Risikofaktor für das Auftreten von ON untersucht (Kawedia et al. 2011). Für die asymptomatischen Läsionen konnte ein höheres Alter als Risiko gefunden werden. Dieses Ergebnis war allerdings statistisch nicht signifikant $(p=$ 0,13). Ein statistisch signifikanter Risikofaktor war das Alter $>10$ Jahre bei den Patienten mit symptomatischen sowie mit schweren ON $(p<0,001)$.

In der GK-MRT-Analyse von Miettunen et al. waren die Kinder mit einem Alter von > 10 Jahren nicht bevorzugt von ON betroffen (Miettunen et al. 2012). Dies lag womöglich an der geringen Anzahl der Patienten mit ON $(n=9)$ in dieser Studie.

Karimova et al. kritisierten, dass überwiegend Studien publiziert wurden, die Patienten mit symptomatischen ON betrachteten und das höhere Alter der Kinder als Risikofaktor für das Auftreten von ON fanden (Karimova et al. 2006). Symptomatisch wären aber nach Karimova et al. vor allem jene Läsionen, die größer sind und diese sind meist erst bei den älteren Kindern zu finden. In der hier vorliegenden Studie wurden sowohl symptomatische wie auch asymptomatische Patienten eingeschlossen und es konnte ein vermehrtes ON-Auftreten in der GK-MRT bei Kindern > 10 Jahren gefunden werden.

Mögliche Gründe für das höhere Risiko der älteren Kinder und Jugendlichen für die Entwicklung von ON sind der Anstieg der Sexualhormone während der Pubertät 
sowie das pubertätsbedingt schnellere Wachstum der Adoleszenten. Durch den Epiphysenfugenschluss steigt der intramedulläre Druck an und der Blutfluss reduziert sich. Daraufhin kommt es im Knochen zur Ischämie mit nachfolgender Nekrose (Kaste et al. 2011). Der unreife Knochen kann aufgrund der noch offenen Wachstumsfuge den erhöhten Druck puffern. Bei den jüngeren Kindern sollte allerdings die Untersuchung auf die Entwicklung von ON nicht vernachlässigt werden (Mattano et al. 2000). In der vorliegenden Studie traten bei 2 Patienten ohne SZT und 5 Patienten mit SZT mit einem Alter von $\leq 10$ Jahren bei der Diagnose der Grunderkrankung bzw. bei der ersten SZT ON in der GK-MRT auf.

\subsubsection{Einflussfaktor Geschlecht auf das Auftreten von Osteonekrosen}

Unter den Patienten ohne SZT wurden bei 38,9 \% der 36 Jungen und bei $44,4 \%$ der 27 Mädchen ON in der GK-MRT detektiert. In der Cox-Regression konnte ein 1,3-mal höheres Risiko für das Auftreten von $\mathrm{ON}$ in der Bildgebung für die weiblichen Patienten gefunden werden $(p=0,477)$. Auch bei den Patienten mit SZT zeigte sich ein 1,6-fach erhöhtes Risiko bei den Mädchen ( $p=0,379)$. Dort wurden bei 10 der 26 weiblichen Patienten sowie bei 7 der 16 männlichen Patienten ON gesehen. Die Ergebnisse beider Analysen waren wie an den p-Werten ersichtlich statistisch nicht signifikant und somit war nur eine Tendenz zum höheren Risiko für das Auftreten von ON in der GK-MRT beim weiblichen Geschlecht erkennbar.

Die Studienlage zum Risikofaktor Geschlecht auf die Entwicklung von ON war nicht einheitlich. In der prospektiven Analyse von Girard et al. waren von den insgesamt 24 Patienten mit in der MRT nachgewiesenen ON genau die Hälfte weiblich (Girard et al. 2013). So entwickelten 2,8 \% der Mädchen und 2,3 \% der Jungen Nekrosen ( $p$ $=0,61)$. Auch in der getrennten Betrachtung der Patientengruppe mit alleiniger Chemotherapie und der Gruppe mit zusätzlicher SZT war das ON-Auftreten unabhängig vom Geschlecht ( $p=0,32$ bzw. $p=0,79$ ).

Jedoch gab es ebenso Studien, bei denen das weibliche Geschlecht mit einem erhöhten Risiko für die Entwicklung von $\mathrm{ON}$ einherging. In der prospektiven Untersuchung von Mattano et al. wurden 2056 Kinder und Jugendliche (1228 männlich, 828 weiblich; Alter 1 - 21 Jahre) mit ALL eingeschlossen (Mattano et al. 2012). ON traten bei 77 Mädchen (10,3\%) und 66 Jungen (6,0 \%) auf und somit signifikant häufiger beim weiblichen Geschlecht ( $p=0,0006)$. Auch Leung et al. fanden ein erhöhtes Risiko für das Auftreten von ON bei Mädchen nach SZT (Leung et al. 2007). Sie untersuchten prospektiv 155 Kinder (82 männlich, 73 weiblich; 
medianes Alter bei SZT: 9,7 Jahre) mit Zustand nach allogener SZT, wobei bei 20 Patienten (13,8 \%) ON auftraten. Die Mädchen zeigten entsprechend der errechneten Hazard Ratio von $H R=3,53$ ein signifikant 3,5-mal höheres Risiko für die Entwicklung von ON als die Jungen ( $p=0,015)$.

Ein möglicher Grund für das häufigere Auftreten der ON beim weiblichen Geschlecht könnten die ansteigenden Östrogenkonzentrationen während der Pubertät darstellen. Neben dem prokoagulatorischen Effekt des Hormons spielt es auch beim Knochenaufbau eine Rolle. Vor allem in der Pubertät steigen die Knochendichte sowie auch die Knochenmasse an. Es kommt bei den Jugendlichen zu einem Ungleichgewicht zwischen dem Blutbedarf des Knochens und dem tatsächlichen Angebot, woraus Mangelerscheinungen in der Trophik resultieren, die letztlich in die Entwicklung von ON übergehen können (Kunstreich et al. 2016). In der vorliegenden Studie konnte kein aussagekräftiges Ergebnis zum genauen Zeitpunkt des Auftretens der ON zwischen den Geschlechtern getroffen und somit verglichen werden, da die Bildgebungen nicht einheitlich und regelmäßig stattfanden. Allerdings war eine Tendenz zu einer früheren Entwicklung der ON beim weiblichen Geschlecht sichtbar. Te Winkel et al. begründeten dies mit dem früheren Eintritt der Mädchen in die Pubertät. Die Hormonkonzentrationen ändern sich und es kommt zum früheren Schluss der Epiphysenfuge (te Winkel et al. 2011).

\subsubsection{Einflussfaktor Steroide auf das Auftreten von Osteonekrosen}

In der vorliegenden Arbeit konnte ein Zusammenhang zwischen der Steroidgabe im Rahmen der Chemotherapie und dem Auftreten von ON in der GK-MRT festgestellt werden. Einer von 23 Patienten, die keine Glukokortikoide erhielten, entwickelte ON. Bei diesem Patient wurde als Grunderkrankung das Osteosarkom diagnostiziert. Dagegen wurden bei 62,5 \% (25/40) der Kinder und Jugendlichen, welche Prednisolon, Dexamethason oder eine Kombination von beiden Medikamenten verabreicht bekamen, ON in der GK-MRT nachgewiesen. Somit unterlagen diese Patienten statistisch signifikant einem 21,6-fach höheren Risiko der Entwicklung von ON gegenüber jenen Kindern und Jugendlichen, die keine Steroide verabreicht bekamen $(p=0,003)$. Auch bei der getrennten Betrachtung der Prednisolon- $(p=$ $0,001)$ sowie Dexamethasondosis $(p=0,642)$ konnte ein erhöhtes Risiko bei den Kindern und Jugendlichen gefunden werden, welche das jeweilige Steroid erhielten. Als Limitation sollte beachtet werden, dass dies eine retrospektive Studie war und die tatsächlich verabreichten kumulativen Steroiddosen gering abweichen könnten. Die 
hier verwendeten Zahlen stammten aus den Protokollen der jeweils passenden Chemotherapie. In den Dokumenten der Patienten war ersichtlich, welche Therapieblöcke und somit auch welche theoretische Dosis an Prednisolon bzw. Dexamethason verabreicht wurden. Allerdings war es möglich, dass einige Kinder und Jugendliche supportiv zusätzlich weitere Steroidgaben erhielten.

In vielen Studien wurde ebenfalls eine Korrelation zwischen einem vermehrten Auftreten von ON bei Patienten, welche Steroide verabreicht bekamen, berichtet. In der Analyse von Girard et al. konnte sowohl in der gesamten Patientenkohorte ( $p<$ 0,001 ) als auch in den beiden Untergruppen (Chemotherapiegruppe: $p=0,027$; SZTGruppen: $p<0,001)$ eine höhere kumulative Steroiddosis mit einem signifikant erhöhten Risiko für ON assoziiert werden (Girard et al. 2013). Dabei nutzten die Autoren das wie in der vorliegenden Arbeit verwendete Prednisolonäquivalent. Die in der Analyse errechneten Schwellenwerte, ab welchen das Risiko für ON stieg, lagen unter allen Patienten bei $>6150 \mathrm{mg} / \mathrm{m}^{2}$, bei den Patienten mit Chemotherapie bei > $5835 \mathrm{mg} / \mathrm{m}^{2}$ und bei den Kindern mit zusätzlicher SZT bei > $2055 \mathrm{mg} / \mathrm{m}^{2}$. Auch McAvoy et al. fanden diesen Zusammenhang in ihrer Analyse von 221 Patienten (141 männlich, 80 weiblich; medianes Alter 28 Jahre) mit allogener oder autologer SZT bei malignen sowie nicht-malignen Grunderkrankungen (McAvoy et al. 2010). Bei 74 Patienten traten ON auf. Die kumulative Steroiddosis dieser Patienten betrug im Median 7043 mg. Bei den Patienten, die keine ON entwickelt hatten, lag die mediane kumulative Steroiddosis bei 1800 mg. Umso höher die verabreichte Dosis war, desto höher lag das ON-Risiko $(p<0,01)$. Diese Aussage konnte von den Autoren auch getroffen haben, als die Gruppen getrennt nach allogener $(p<0,01)$ und autologer SZT $(p=0,03)$ ausgewertet wurden. Elmantaser et al. schlossen in ihre retrospektive Studie 186 Kinder (110 männlich, 76 weiblich; mittleres Alter 5,3 Jahre) mit ALL ein (Elmantaser et al. 2010). Sie konnten zeigen, dass die Inzidenz der ON bei Patienten, die Dexamethason erhielten, mit $11 \%$ höher war als jene mit 3,5 \% bei den Kindern, welche Prednisolon verabreicht bekamen. Dies konnte in der vorliegenden Arbeit aufgrund der geringen Anzahl der Patienten ( $n=5)$, die nur Dexamethason erhielten, nicht aussagekräftig dargestellt werden.

Mattano et al. untersuchten in ihrer prospektiven Analyse den Einfluss von alternierender im Vergleich zu kontinuierlicher Dexamethasongabe auf das Auftreten von ON bei Kindern und Jugendlichen mit ALL (Mattano et al. 2012). Bei 143 Patienten konnten Läsionen anhand von klinischer und radiologischer Diagnostik 
festgestellt werden. Die Nekrosen traten häufiger (17\%) und früher (14,0 Jahre) bei den Patienten mit kontinuierlicher im Vergleich zu den Patienten mit alternierender Steroidgabe (8,7 \%; 16,2 Jahre) auf. Die erhöhte Rate an ON bei den Kindern und Jugendlichen mit kontinuierlicher Medikamentengabe war statistisch signifikant $(p<$ 0,001). Die Autoren begründeten dieses Ergebnis darin, dass sich durch die Steroidpause der Knochen vom erhöhten intramedullären Druck erholen kann und somit die Apoptose der Osteozyten reduziert wird, wodurch sich weniger Nekrosen entwickeln.

Die Gabe von Steroiden im Rahmen der Chemotherapie bzw. nach SZT muss als wichtiger Risikofaktor für das Auftreten von ON berücksichtigt werden. Vor allem in der Therapie der Leukämie oder der Lymphome sowie bei der Behandlung der Graftversus-Host-Disease nach SZT sind Steroide essentiell und es gibt keine gute Alternativmedikation (Karimova et al. 2007). Schon 3 Monate nach Beginn einer Steroidtherapie konnten in der MRT mit Hilfe der STIR-Sequenz ON detektiert werden (Kubo et al. 2015). Die meisten ON entstanden innerhalb eines Jahres (Kaste et al. 2015). Aufgrund dieses frühen Auftretens der Nekrosen nach Beginn der Steroidgabe sollten betroffene Patienten gründlich untersucht werden.

\subsubsection{Verlauf der Osteonekrosen}

Für die Verlaufsbeurteilung wurden 15 Patienten mit Morbus Hodgkin und ohne SZT eingeschlossen. Dies war hinsichtlich der Grunderkrankung und ihrer Behandlung eine relativ homogene Gruppe, da unter den Hodgkin-Patienten die meisten Patienten vorgefunden werden konnten, bei welchen ON in der GK-MRT zu sehen und bei denen auch im Verlauf GK-MRT-Untersuchungen vorhanden waren. Es wurde für diese Gruppe überwiegend eine Abnahme der Summe der ON-Volumina im zeitlichen Verlauf beobachtet.

Nur bei 2 von 7 Kindern und Jugendlichen, die keine Behandlung hinsichtlich ihrer ON erhielten, konnte eine Zunahme des Gesamtvolumens aller vorhandenen ON verzeichnet werden. Der Beobachtungszeitraum für diese beiden Patienten betrug 9 bzw. 15 Monate nach dem erstmaligen Auftreten von ON in der GK-MRT. Anzumerken ist, dass bei diesen beiden Patienten im gesamten Verlauf keine Schmerzsymptomatik dokumentiert wurde. 3 Patienten von der Gruppe der Kinder und Jugendlichen ohne Therapie ihrer $\mathrm{ON}$, die initial schon symptomatisch waren bzw. im Verlauf wurden oder blieben, zeigten alle eine Abnahme der Summe der ON-Volumina. 
Unter den 8 Patienten, welche zu unterschiedlichen Zeitpunkten im Beobachtungszeitraum eine Behandlung bezüglich ihrer ON erhielten, konnte schon vor Beginn der Therapie bei 7 Patienten eine Abnahme des Gesamtvolumens aller ON verzeichnet werden. Lediglich bei einer Patientin war eine Zunahme zu beobachten. Diese Patientin blieb im gesamten Verlauf asymptomatisch. Unter lloprosttherapie nahm die Summe der ON-Volumina dann auch ab. Auch bei 6 weiteren Patienten nahm das Gesamtvolumen aller aufgetretenen ON unter der Therapie im jeweils beobachteten Zeitraum ab. Bei einem Patienten in dieser Gruppe konnte eine Gesamtvolumenzunahme unter ON-Therapie mittels Anbohrung und zusätzlicher lloprostgabe von lediglich $2 \mathrm{ml}$ gesehen werden. Auch dieser Patient beklagte, wie alle anderen Kinder und Jugendlichen bei denen eine Zunahme des Volumens aller ON verzeichnet wurde, keine Knochenschmerzen.

Zusammenfassend war festzustellen, dass in dieser untersuchten Patientengruppe sowohl ohne Therapie als auch mit Behandlung hinsichtlich der ON eine überwiegende Tendenz zur Regression der ON-Volumina bestand. Unter den 8 Patienten mit einer Therapie konnte keine Aussage darüber gemacht werden, bei welcher Behandlung das Volumen am meisten sank. Sowohl bei den Patienten mit alleiniger lloprosttherapie sowie in Kombination mit Zoledronat oder einer Anbohrung wurde eine Abnahme des Gesamtvolumens der ON von über $50 \%$ beobachtet. Auch bei der alleinigen supportiven Behandlung mit Vitamin $D$ und Calcium konnte eine Regression der Summe der ON-Volumina von um die 50 \% gesehen werden. Die geringste Abnahme mit 38 \% wurde unter einer Therapie mit lloprost in Kombination mit Vitamin $\mathrm{D}$ und Calcium verzeichnet, allerdings betrug bei diesem Patienten die Beobachtungszeit der ON nur 3 Monate.

Niinimäki et al. untersuchten in ihrer prospektiven Studie 79 Kinder (51 männlich, 46 weiblich; mittleres Alter bei Diagnose ALL: 6,4 Jahre) mit ALL (Niinimaki et al. 2007). 23 Patienten entwickelten in den MRT-Aufnahmen sichtbare ON in der unteren Extremität. Für 10 Patienten wurden über 1 bis 8 Jahre nach Chemotherapieende Verlaufs-MRT-Untersuchungen durchgeführt. Unter den 9 asymptomatischen Kindern wurden zum Verlaufsende bei einem Patienten keine ON mehr gesehen und bei den restlichen 8 Patienten waren neben stabilen Verläufen der Größe der ON auch Abnahmen zu verzeichnen. Lediglich bei einem symptomatischen Patienten kam es zur Progression der ON mit einem nachfolgend notwendigen bilateralen Hüftersatz. Daraus schlussfolgerten die Autoren, dass die Bildgebung im Verlauf nur 
bei symptomatischen Patienten sinnvoll sei. Ebenfalls verschlechterten sich im Verlauf nur die ON des Femurkopfes, die möglicherweise eine chirurgische Behandlung erforderlich machen.

Kaste et al. schlossen in ihre prospektive Studie 462 Kinder mit ALL ein, bei denen MRT-Aufnahmen der Hüfte durchgeführt wurden, um schwere ON, d.h. Läsionen, die $>30 \%$ der Gelenkfläche betreffen, zu detektieren (Kaste et al. 2015). Innerhalb eines Jahres nach Beginn der Steroidtherapie entwickelten $79 \%$ der Patienten ON. Die Autoren sahen ein weiteres Screening von asymptomatischen Patienten als unnötig, wenn diese nach diesem einem Jahr keine $\mathrm{ON}$ in der Hüfte aufwiesen. Nur bei 4,6 \% der Patienten konnten nach diesem Zeitraum neue Läsionen entdeckt werden. In dieser Studie wurde ebenfalls gezeigt, dass auch die hier definierten schweren ON eine spontane Regression zeigten. Bei 50 \% der 8 Hüft-ON der Kinder im Alter von $\leq 10$ Jahren wurden im Verlauf keine Läsionen mehr gesehen. Hingegen fand sich bei den 34 Hüft-ON der Patienten im Alter von > 10 Jahren kein solcher Verlauf.

Laut diesen beiden Studien sollten asymptomatische Patienten, nachdem bei innen ON nachgewiesen worden sind, im weiteren Krankheitsverlauf keine MRTVerlaufsuntersuchungen erhalten. Diese Aussage konnte mit den Ergebnissen aus der vorliegenden Studie nicht bestätigt werden. Auch wenn die Zunahme der ONVolumina sich nur gering hielten bzw. die Bildgebungen nur für einen kurzen Beobachtungszeitraum verfügbar waren, sind die progressiven Verläufe der Entwicklung und Ausdehnung von ON nur bei jenen Patienten aufgetreten, die im Verlauf nie über Knochenschmerzen klagten. Somit sollten sowohl symptomatische als auch asymptomatische Patienten hinsichtlich des Verlaufs der ON Kontrollbildgebungen erhalten. Entgegen der beiden zum Vergleich vorgestellten Studien ist die Aufnahme des gesamten Körpers sinnvoll, um alle ON im Skelett ausfindig zu machen, vor allem auch die asymptomatischen $\mathrm{ON}$, welche die progressiven Verläufe zeigten und später eventuell zu Schmerzsymptomatik mit notwendiger chirurgischer Intervention führen könnten.

In der vorliegenden Arbeit wurden Patienten mit unterschiedlichen Behandlungen der ON dargestellt. Die Infusionstherapie mit dem Prostacyclinanalogon Iloprost als offlabel-Therapiemöglichkeit in der Behandlung der ON diente der Vasodilatation. Vor allem die Mikrozirkulation im Nekroseareal sollte dadurch verbessert werden. 
Weiterhin reduzierte es die kapillare Durchlässigkeit und hemmte die Thrombozytenaggregation (Jager et al. 2009). Mehrere Autoren belegten die positive Wirkung des lloprosts hinsichtlich der Verbesserung der Gelenkfunktion und der Abnahme des Schmerzes und somit der steigenden Zufriedenheit der Patienten (Disch et al. 2005, Jager et al. 2009). Auch in der MRT konnte eine Abnahme der Läsionsgröße beim Knochenmarködem, jedoch nicht konsequent bei fortgeschrittenen ON, nachgewiesen werden (Disch et al. 2005). Ebenfalls zur Therapie von frühen Stadien der ON können im off-label-use Bisphosphonate eingesetzt werden. In einer vorhandenen Nekrose schreitet die Knochenresorption durch die Osteoklasten fort. Dieser Vorgang soll durch die Gabe von z.B. Alendronat oder Zoledronat gehemmt werden, um ein Fortschreiten der ON bis zum Gelenkeinbruch oder zur Fraktur zu verhindern. Für diese antiresorptiven Substanzen wurde ebenfalls ein Schmerzrückgang sowie eine geringe Zeitverzögerung bis zum Gelenkeinbruch geschildert (Roth et al. 2015). Padhye et al. beschrieben im Verlauf eine Konstanz des Ausmaßes der ON in Knie-, Sprung- und Schultergelenk unter Zoledronattherapie, im Gegensatz dazu aber ein Progress der ON des Hüftgelenks mit teils notwendigem operativem Gelenkersatz (Padhye et al. 2016). Als operative Intervention diente die Anbohrung von ON der Entlastung des erhöhten intraossären Drucks sowie der venösen Stauung. Die Mikrozirkulation wurde auch hier verbessert und dadurch das Knochenremodelling ermöglicht (Jager et al. 2009).

Beckmann et al. verglichen die Wirkung von lloprost (12 Patienten: 9 männlich, 3 weiblich; mittleres Alter 37 Jahre), Anbohrung (12 Patienten: 8 männlich, 4 weiblich; mittleres Alter 36,3 Jahre) oder eine Kombination von beiden Therapien (12 Patienten: 9 männlich, 3 weiblich; mittleres Alter 37,7 Jahre) auf die weitere Entwicklung von Knochenmarködemen und frühen $\mathrm{ON}$ im proximalen Femur (Beckmann et al. 2013). Sowohl unter der lloprostinfusion als auch in den ersten postoperativen Tagen nach einer Anbohrung trat eine Schmerzreduktion auf. Die Werte von unterschiedlichen klinischen Scores verbesserten sich signifikant 3 und 12 Monate nach der Therapie $(p<0,001)$. Nach 12 Monaten konnte eine signifikant deutlichere Verbesserung der klinischen Scores bei den Patienten mit der Kombinationstherapie im Vergleich zu jenen mit den Monotherapien eruiert werden ( $p<0,03$ bzw. $p<0,001$ ). In der MRT nahm die Größe der ON unter der Behandlung mittels Anbohrung bei 3 Patienten ab, bei einem Patienten mit der 
Kombinationstherapie fand sich eine komplette Regression und bei keinem der Patienten mit der alleinigen lloprostbehandlung konnte solch ein Verlauf gesehen werden. 12 Monate nach der jeweiligen Therapie traten in den Gruppen mit den Monotherapien zusätzliche ON auf. Die Autoren schlossen daraus, dass die lloprostbehandlung sowie die Anbohrung allein vergleichbare Ergebnisse zeigten. Die Kombinationstherapie war am aussichtsreichsten und zuverlässigsten bei der Therapie von Knochenmarködemen und frühen ON.

Allerdings sollte beachtet werden, dass der Effekt eines chirurgischen Eingriffes wie z.B. der Anbohrung schwer beurteilbar ist, wenn die ON multifokal im Patienten auftreten. So konnte in der vorliegenden Studie die Zuverlässigkeit der Anbohrung schwer beurteilt werden, zudem bei allen betrachteten Patienten, die solch eine Behandlung erhielten, zusätzlich lloprost mit systemischer Wirkung verabreicht wurde.

In aktuellen Studien wurde als neue Therapievariante die Verknüpfung von Markraumdekompression mit zusätzlicher Knochentransplantation bewertet. Diese führte laut Lazik et al. in der MRT-Kontrolle zur Reduktion des ON-Volumens mit einer Korrelation zur Verbesserung der klinischen Symptome (Lazik et al. 2015). Bei klinisch stummen Läsionen war die Größenverminderung deutlicher als bei symptomatischen ON. Mit Hilfe der MRT konnte der Remodellingprozess vom avitalen Transplantat mit geringer Signalintensität zum vitalen Gewebe durch zunehmende Vaskularisation veranschaulicht werden. Die steigende Signalintensität diente als Vitalitätszeichen und somit als Marker für die Heilungsreaktion. In den Metaanalysen von Lau et al. und Papakostidis et al. wurde ebenfalls die Kombination aus Anbohrung und Stammzellgabe gegenüber der konventionellen Markraumdekompression bei ON des Femurkopfs aufgrund von Verbesserung von klinisch funktionellen Scores, Verminderung des Schmerzes und geringeren Gelenkeinbruchraten favorisiert (Lau et al. 2014, Papakostidis et al. 2016).

Te Winkel et al. fassten in einem Überblick zu vorhandener Literatur zu Behandlungsoptionen von ON bei an ALL erkrankten Kindern und Jugendlichen zusammen, dass es schwer zu beurteilen war, welche die beste Therapie sei, da es noch an qualitativ guten Studien mangelte (Te Winkel et al. 2014). So konnte auch mit der vorliegenden Arbeit keine Aussage dazu getroffen werden, da die Patientenzahlen sehr klein waren und die Dauer der Beobachtungszeiträume stark variierte. Zudem erhielten die Patienten viele verschiedene Behandlungen zu 
unterschiedlichen Zeitpunkten, in variierender Dosierung und Dauer. Schon in dieser kleinen Gruppe von Patienten wurden mehrere Behandlungsmöglichkeiten versucht. Daran ist zu erkennen, dass es aktuell noch keinen Standard gibt, welche Therapie wann am besten eingesetzt werden sollte.

Orthopäden, welche die in der vorliegenden Arbeit an ON erkrankten Kinder und Jugendlichen mitbetreuten und die Therapieentscheidung mit trafen, waren sich auch unschlüssig, welche Therapie effektiver sei. Sinnvoll waren neben lloprost, Anbohrung und Bisphosphonaten auch die Physiotherapie mit Bewegung ohne Belastung, eine Procainiontophorese, Neural- und Magnetfeldtherapien, Plexusblockaden etc. An erster Stelle der Therapieindikation stand der Schmerz. Es gab keine sichere Grenze, ab wann z.B. Iloprost oder zusätzlich Bisphosphonate eingesetzt werden sollten. Aus den Erfahrungen heraus sei der Einsatz von Iloprost großzügiger geworden. Gute Ergebnisse konnten vor allem nach Knochenprellungen im Sportbereich verzeichnet werden. Deswegen sollten laut den Orthopäden alle Patienten mit Durchblutungsstörungen des Knochens, egal welcher Genese, lloprost als Therapievariante angeboten bekommen. Gelenknähe der ON mit Frakturgefahr, eine übersichtliche Anzahl der Läsionen, Zunahme der Befunde im Vergleich oder einfach die fehlenden Alternativen indizierten eine chirurgische Intervention. So wurde dann eine Anbohrung in Kombination mit einer lloprosttherapie durchgeführt. Bei geringgradigen, weitverteilten und/oder an schwierigen Stellen liegenden ON blieb nur die alleinige medikamentöse Therapie.

\subsection{Limitationen der Studie und Ausblick}

Limitationen der vorliegenden retrospektiven Arbeit waren zum einen die niedrige Patientenzahl insgesamt als auch die Anzahl der Patienten mit ON. Auch wenn die Anzahl der eingeschlossenen Patienten mit ON höher als in den bislang publizierten Studien zur GK-MRT von ON war, ließen vor allem die vielen unterschiedlichen Grunderkrankungen keine eindeutigen Ergebnisse zu.

Aufgrund des retrospektiven Formats der Studie konnten keine einheitlichen Zeitpunkte zur Durchführung einer GK-MRT-Untersuchung vorgegeben werden. So existierte nicht bei jedem Patienten eine Aufnahme zur Diagnosestellung der Grunderkrankung und auch wenn später eine Bildgebung durchgeführt wurde, war der Abstand zum Zeitpunkt der Diagnose sehr verschieden. Vor allem bei den betrachteten Verläufen der ON über die Zeit fand sich nicht immer eine GK-MRT- 
Aufnahme im halbjährlichen Intervall nach erstmaligem kernspintomographischem Auftreten der ON. Somit war der Vergleich zwischen den Patienten im longitudinalen Verlauf schwierig.

Für die Beurteilung der Risikofaktoren wurde nicht der Zeitpunkt des Auftretens der ON im Körper des Patienten, sondern der erstmalige Nachweis in der GK-MRT als Bezugspunkt gewählt. Es war durchaus möglich, dass die $\mathrm{ON}$ schon früher aufgetreten waren bzw. auch in einer MRT-Aufnahme zu sehen waren.

Für das bessere Verstehen des natürlichen Verlaufs von ON aber auch die Reaktion der Läsionen auf eine Therapie sollten zukünftig prospektive GK-MRT-Studien durchgeführt werden. Mit entsprechenden GK-MRT-Untersuchungen in konstanten Zeitintervallen in Bezug zum Zeitpunkt der Diagnosestellung der Grunderkrankung sowie mit homogeneren und größeren Patientengruppen könnten nicht nur die Risikofaktoren sondern z.B. auch die auftretende Symptomatik in Bezug zur Größe einer ON verifiziert werden. Zur Realisierung eines entsprechend größeren Stichprobenumfanges werden multizentrische Studien empfohlen, bei denen die GKMRT mit einem vorgegebenen standardisierten Untersuchungsprotokoll erfolgen sollte. Um die Effektivität einer Therapie zur Behandlung aufgetretener ON zu beurteilen, braucht es qualitativ gute Studien, in denen z.B. mit Kontrollgruppen der Nutzen der Prostacyclin- oder Bisphosphonat-Behandlung in einer einheitlichen Dosierung und gleicher Zeitdauer der Verabreichung analysiert werden kann. Eventuell könnte durch Charakterisierung von frühen Zeichen der ON in der MRT die Häufigkeit des Auftretens von symptomatischen Nekrosen reduziert werden.

Ebenfalls sollte die Aufteilung der Patienten mit SZT hinsichtlich des Spendertyps erfolgen. Autologe SZT gelten als Therapieintensivierung ohne immunologische Einflüsse auf den Knochen. Im Gegensatz dazu ist die Immunologie bei den Patienten, welche eine allogene SZT erhielten, wichtig. ON könnten als Ausdruck einer GvHD des Knochens gelten. Auch bei anderen Lokalisationen der GvHD erfolgt die Behandlung mit Steroiden. Somit könnten dadurch vermehrt ON in dieser Patientengruppe auftreten. Dieses Problem sollte in einer sich an diese Arbeit anschließenden Studie untersucht werden.

Eine weitere Limitation war das ausgewählte Sequenzprotokoll. Insbesondere bei onkologischen Erkrankungen sollten diffusionswichtende Sequenzen eingesetzt werden, die insbesondere Akutveränderungen sicher aufzeigen können. In Nachfolgestudien ist ein Einschluss der GK-DWI in axialer Orientierung erforderlich. 


\section{Schlussfolgerung}

Die Ergebnisse der vorliegenden Arbeit und die aktuelle Literatur zeigen, das mit Hilfe der GK-MRT ON als Komplikation bei onkologisch oder hämatologisch erkrankten Kindern und Jugendlichen sicher detektiert werden. Laut der vorliegenden Studie sollten dafür aufgrund der sehr guten Darstellung und Eindeutigkeit der Einschätzung dieser Läsionen sowie deren Ausdehnung in Bezug auf den Markraum und die Gelenkflächen insbesondere die T1-SPACE-Sequenz in Verlaufskontrollen genutzt werden. Zur Beurteilung der Grunderkrankung und anderer Komplikationen sind in der primären Untersuchung allerdings weitere Sequenzen erforderlich und das Sequenzprotokoll ist an die jeweilige Fragestellung anzupassen.

Patienten, bei denen die analysierten Einflussfaktoren für ein höheres Risiko des Auftretens von ON in der GK-MRT wie ein Alter > 10 Jahre zum Zeitpunkt der Diagnosestellung der Grunderkrankung oder bei der ersten SZT und möglicherweise auch das weibliche Geschlecht vorliegen, sind $\mathrm{zu}$ identifizieren und intensiver $\mathrm{zu}$ beobachten. Insbesondere ist auf die verabreichte kumulative Steroiddosis, welche die Patienten teils im Rahmen des Chemotherapieprotokolls zur Behandlung der jeweiligen Grunderkrankung bzw. im Rahmen der SZT erhalten, zu achten. Patienten, die Prednisolon oder Dexamethason bekommen, unterliegen einem erheblich größeren Risiko der Entwicklung von ON.

Regelmäßig stattfindende GK-MRT-Untersuchungen können asymptomatische und multifokal auftretende Läsionen aufdecken. Dadurch könnten schon frühzeitig Veränderungen in der Bildgebung als Hinweis auf eine sich entwickelnde ON verifiziert werden und somit die schweren Verläufe mit notwendiger chirurgischer Intervention den pädiatrischen Patienten zukünftig erspart bleiben. 


\section{Literaturverzeichnis}

Albano D, Patti C, La Grutta L, Grassedonio E, Mule A, Brancatelli G, Lagalla R, Midiri M, Galia M. 2017. Osteonecrosis detected by whole body magnetic resonance in patients with Hodgkin Lymphoma treated by BEACOPP. Eur Radiol, 27 (5):21292136.

Assouline-Dayan Y, Chang C, Greenspan A, Shoenfeld Y, Gershwin ME. 2002.

Pathogenesis and natural history of osteonecrosis. Semin Arthritis Rheum, 32 (2):94124.

Beckmann J, Schmidt T, Schaumburger J, Rath B, Luring C, Tingart M, Grifka J. 2013. Infusion, core decompression, or infusion following core decompression in the treatment of bone edema syndrome and early avascular osteonecrosis of the femoral head. Rheumatol Int, 33 (6):1561-1565.

Beer M, Stenzel M, Girschick H, Schlegel PG, Darge K. 2008. [Whole-body MR imaging in children with suspected osteonecrosis after intensive chemotherapy: preliminary results]. Rofo, 180 (3):238-245.

Burger B, Beier R, Zimmermann M, Beck JD, Reiter A, Schrappe M. 2005. Osteonecrosis: a treatment related toxicity in childhood acute lymphoblastic leukemia (ALL)-experiences from trial ALL-BFM 95. Pediatr Blood Cancer, 44 (3):220-225.

Campbell S, Sun CL, Kurian S, Francisco L, Carter A, Kulkarni S, Parker P, Karanes C, Forman SJ, Bhatia S. 2009. Predictors of avascular necrosis of bone in long-term survivors of hematopoietic cell transplantation. Cancer, 115 (18):4127-4135.

Chavhan GB, Babyn PS. 2011. Whole-body MR imaging in children: principles, technique, current applications, and future directions. Radiographics, 31 (6):1757-1772.

Darge K, Jaramillo D, Siegel MJ. 2008. Whole-body MRI in children: current status and future applications. Eur J Radiol, 68 (2):289-298.

den Hoed MA, Pluijm SM, te Winkel ML, de Groot-Kruseman HA, Fiocco M, Hoogerbrugge $P$, Leeuw JA, Bruin MC, van der Sluis IM, Bresters D, Lequin MH, Roos JC, Veerman AJ, Pieters R, van den Heuvel-Eibrink MM. 2015. Aggravated bone density decline following symptomatic osteonecrosis in children with acute lymphoblastic leukemia. Haematologica, 100 (12):1564-1570.

Disch AC, Matziolis G, Perka C. 2005. The management of necrosis-associated and idiopathic bone-marrow oedema of the proximal femur by intravenous iloprost. J Bone Joint Surg Br, 87 (4):560-564.

Duncan CN, Majhail NS, Brazauskas R, Wang Z, Cahn JY, Frangoul HA, Hayashi RJ, Hsu JW, Kamble RT, Kasow KA, Khera N, Lazarus HM, Loren AW, Marks DI, Maziarz RT, Mehta P, Myers KC, Norkin M, Pidala JA, Porter DL, Reddy V, Saber W, Savani BN, Schouten HC, Steinberg A, Wall DA, Warwick AB, Wood WA, Yu LC, Jacobsohn DA, Sorror ML. 2015. Long-term survival and late effects among one-year survivors of second allogeneic hematopoietic cell transplantation for relapsed acute leukemia and myelodysplastic syndromes. Biol Blood Marrow Transplant, 21 (1):151-158.

Elmantaser M, Stewart G, Young D, Duncan R, Gibson B, Ahmed SF. 2010. Skeletal morbidity in children receiving chemotherapy for acute lymphoblastic leukaemia. Arch Dis Child, 95 (10):805-809.

Engelbrecht V, Scherer A, Bruder M, Korholz D, Modder U. 2000. [MRI of aseptic osteonecrosis in children and adolescents with acute lymphoblastic leukemia]. Rofo, 172 (4):336-341.

Enrici RM, Anselmo AP, Donato V, Santoro M, Tombolini V. 1998. Avascular osteonecrosis in patients treated for Hodgkin's disease. Eur J Haematol, 61 (3):204-209.

Faraci M, Calevo MG, Lanino E, Caruso S, Messina C, Favr C, Iori A, Santaron S, Bonanomi S, Rondelli R, Dini G, Haupt R, Group A-S. 2006. Osteonecrosis after allogeneic stem cell transplantation in childhood. A case-control study in Italy. Haematologica, 91 (8):1096-1099.

Flouzat-Lachaniette CH, Roubineau F, Heyberger C, Bouthors C, Hernigou P. 2016. Multifocal osteonecrosis related to corticosteroid: ten years later, risk of progression and observation of subsequent new osteonecroses. Int Orthop, 40 (4):669-672. 
Gaa J. 2006. Ganzkörper-MR-Tomographie. In: Rummeny EJ, Reimer P, Heindel W, Hrsg. Ganzkörper-MR-Tomographie. Zweite Aufl. Stuttgart: Georg Thieme Verlag KG.

Gaynon PS. 2015. ALL and osteonecrosis. Blood, 126 (15):1734-1735.

Geczova L, Soltysova A, Gecz J, Sufliarska S, Horakova J, Mladosievicova B. 2015. Avascular necrosis of bone in childhood cancer patients: a possible role of genetic susceptibility. Bratisl Lek Listy, 116 (5):289-295.

Giesecke M. 2002. WaKo3D - Wahrnehmung und Kommunikation: Training und theoretische Grundlagen kommunikativer Schlüsselfunktionen: Selbstlernmedium. URL: http://www.michael-giesecke.de/wako3d/texte/01_wahrnehmen/schema/ koerperwahrnehmung_1.htm (Stand: 21.08.2017).

Girard P, Auquier P, Barlogis V, Contet A, Poiree M, Demeocq F, Berbis J, Herrmann I, Villes V, Sirvent N, Kanold J, Chastagner P, Chambost H, Plantaz D, Michel G. 2013. Symptomatic osteonecrosis in childhood leukemia survivors: prevalence, risk factors and impact on quality of life in adulthood. Haematologica, 98 (7):1089-1097.

Goo HW. 2015. Whole-Body MRI in Children: Current Imaging Techniques and Clinical Applications. Korean J Radiol, 16 (5):973-985.

Goo HW, Choi SH, Ghim T, Moon HN, Seo JJ. 2005. Whole-body MRI of paediatric malignant tumours: comparison with conventional oncological imaging methods. Pediatr Radiol, 35 (8):766-773.

Inaba H, Pui CH. 2010. Glucocorticoid use in acute lymphoblastic leukaemia. Lancet Oncol, 11 (11):1096-1106.

Ippolito D, Masetto A, Franzesi CT, Bonaffini PA, Sala A, Biondi A, Sironi S. 2016. Lowerlimb MRI in the staging and re-staging of osteonecrosis in paediatric patients affected by acute lymphoblastic leukaemia after therapy. Skeletal Radiol, 45 (4):495-503.

Jager M, Zilkens C, Westhoff B, Jelinek EM, Kozina G, Krauspe R. 2009. Efficiency of iloprost treatment for chemotherapy-associated osteonecrosis after childhood cancer. Anticancer Res, 29 (8):3433-3440.

Jaramillo D. 2009. What is the optimal imaging of osteonecrosis, Perthes, and bone infarcts? Pediatr Radiol, 39 Suppl 2:S216-219.

Karimova EJ, Wozniak A, Wu J, Neel MD, Kaste SC. 2010. How does osteonecrosis about the knee progress in young patients with leukemia?: a 2- to 7-year study. Clin Orthop Relat Res, 468 (9):2454-2459.

Karimova EJ, Rai SN, Howard SC, Neel M, Britton L, Pui CH, Kaste SC. 2007. Femoral head osteonecrosis in pediatric and young adult patients with leukemia or lymphoma. J Clin Oncol, 25 (12):1525-1531.

Karimova EJ, Rai SN, Ingle D, Ralph AC, Deng X, Neel MD, Howard SC, Pui CH, Kaste SC. 2006. MRI of knee osteonecrosis in children with leukemia and lymphoma: Part 2, clinical and imaging patterns. AJR Am J Roentgenol, 186 (2):477-482.

Karol SE, Mattano LA, Jr., Yang W, Maloney KW, Smith C, Liu C, Ramsey LB, Fernandez CA, Chang TY, Neale G, Cheng C, Mardis E, Fulton R, Scheet P, San Lucas FA, Larsen EC, Loh ML, Raetz EA, Hunger SP, Devidas M, Relling MV. 2016. Genetic risk factors for the development of osteonecrosis in children under age 10 treated for acute lymphoblastic leukemia. Blood, 127 (5):558-564.

Karol SE, Yang W, Van Driest SL, Chang TY, Kaste S, Bowton E, Basford M, Bastarache L, Roden DM, Denny JC, Larsen E, Winick N, Carroll WL, Cheng C, Pei D, Fernandez CA, Liu C, Smith C, Loh ML, Raetz EA, Hunger SP, Scheet P, Jeha S, Pui CH, Evans WE, Devidas M, Mattano LA, Jr., Relling MV. 2015. Genetics of glucocorticoidassociated osteonecrosis in children with acute lymphoblastic leukemia. Blood, 126 (15):1770-1776.

Kaste SC, Karimova EJ, Neel MD. 2011. Osteonecrosis in children after therapy for malignancy. AJR Am J Roentgenol, 196 (5):1011-1018.

Kaste SC, Pei D, Cheng C, Neel MD, Bowman WP, Ribeiro RC, Metzger ML, Bhojwani D, Inaba H, Campbell P, Rubnitz JE, Jeha S, Sandlund JT, Downing JR, Relling MV, Pui $\mathrm{CH}$, Howard SC. 2015. Utility of early screening magnetic resonance imaging for extensive hip osteonecrosis in pediatric patients treated with glucocorticoids. J Clin Oncol, 33 (6):610-615. 
Kawedia JD, Kaste SC, Pei D, Panetta JC, Cai X, Cheng C, Neale G, Howard SC, Evans WE, Pui CH, Relling MV. 2011. Pharmacokinetic, pharmacodynamic, and pharmacogenetic determinants of osteonecrosis in children with acute lymphoblastic leukemia. Blood, 117 (8):2340-2347; quiz 2556.

Klee D, Riccabona M, Born M, Leenen A, von Kalle T, Mentzel HJ, Körber F, Heyer C. 2017. Informationen der Gesellschaft für Pädiatrische Radiologie zur Verwendung von gadoliniumhaltigem Kontrastmittel für die Kernspintomografie im Kindes- und Jugendalter. Rofo, 189 (9):817-819.

Korholz D, Bruder M, Engelbrecht V, Ruther W, Gobel U. 1998. Aseptic osteonecrosis in children with acute lymphoblastic leukemia. Pediatr Hematol Oncol, 15 (4):307-315.

Krohmer S, Sorge I, Krausse A, Kluge R, Bierbach U, Marwede D, Kahn T, Hirsch W. 2010. Whole-body MRI for primary evaluation of malignant disease in children. Eur J Radiol, 74 (1):256-261.

Kubo Y, Yamamoto T, Motomura G, Tsukamoto N, Karasuyama K, Sonoda K, Hatanaka H, Utsunomiya T, Iwamoto Y. 2015. MRI-detected bone marrow changes within 3 weeks after initiation of high-dose corticosteroid therapy: a possible change preceding the subsequent appearance of low-intensity band in femoral head osteonecrosis. Rheumatol Int, 35 (11):1909-1912.

Kunstreich M, Kummer S, Laws HJ, Borkhardt A, Kuhlen M. 2016. Osteonecrosis in children with acute lymphoblastic leukemia. Haematologica, 101 (11):1295-1305.

Lackner H, Benesch M, Moser A, Smolle-Juttner F, Linhart W, Raith J, Urban C. 2005. Aseptic osteonecrosis in children and adolescents treated for hemato-oncologic diseases: a 13-year longitudinal observational study. J Pediatr Hematol Oncol, 27 (5):259-263.

Laffan EE, O'Connor R, Ryan SP, Donoghue VB. 2004. Whole-body magnetic resonance imaging: a useful additional sequence in paediatric imaging. Pediatr Radiol, 34 (6):472-480.

Lau RL, Perruccio AV, Evans HM, Mahomed SR, Mahomed NN, Gandhi R. 2014. Stem cell therapy for the treatment of early stage avascular necrosis of the femoral head: a systematic review. BMC Musculoskelet Disord, 15:156.

Lazik A, Landgraeber S, Classen T, Kraff O, Lauenstein TC, Theysohn JM. 2015. Aspects of postoperative magnetic resonance imaging of patients with avascular necrosis of the femoral head, treated by advanced core decompression. Skeletal Radiol, 44 (10):1467-1475.

Leung W, Ahn H, Rose SR, Phipps S, Smith T, Gan K, O'Connor M, Hale GA, Kasow KA, Barfield RC, Madden RM, Pui CH. 2007. A prospective cohort study of late sequelae of pediatric allogeneic hematopoietic stem cell transplantation. Medicine (Baltimore), $86(4): 215-224$.

Ley S, Ley-Zaporozhan J, Schenk JP. 2009. Whole-body MRI in the pediatric patient. Eur J Radiol, 70 (3):442-451.

Li X, Brazauskas R, Wang Z, Al-Seraihy A, Baker KS, Cahn JY, Frangoul HA, Gajewski JL, Hale GA, Hsu JW, Kamble RT, Lazarus HM, Marks DI, Maziarz RT, Savani BN, Shah AJ, Shah N, Sorror ML, Wood WA, Majhail NS. 2014. Avascular necrosis of bone after allogeneic hematopoietic cell transplantation in children and adolescents. Biol Blood Marrow Transplant, 20 (4):587-592.

Link TM. 2006. Avaskuläre Nekrosen. In: Rummeny EJ, Reimer P, Heindel W, Hrsg. Ganzkörper-MR-Tomographie. Zweite Aufl. Stuttgart: Georg Thieme Verlag KG.

Littooij AS, Kwee TC, Enriquez G, Verbeke JI, Granata C, Beishuizen A, de Lange C, Zennaro F, Bruin MC, Nievelstein RA. 2017. Whole-body MRI reveals high incidence of osteonecrosis in children treated for Hodgkin lymphoma. $\mathrm{Br} \mathrm{J}$ Haematol, 176 (4):637-642.

Liu Y, Jiang W, Liu S, Su X, Zhou S. 2015. Combined effect of tnf-alpha polymorphisms and hypoxia on steroid-induced osteonecrosis of femoral head. Int J Clin Exp Pathol, 8 (3):3215-3219. 
Mattano LA, Jr., Sather HN, Trigg ME, Nachman JB. 2000. Osteonecrosis as a complication of treating acute lymphoblastic leukemia in children: a report from the Children's Cancer Group. J Clin Oncol, 18 (18):3262-3272.

Mattano LA, Jr., Devidas M, Nachman JB, Sather HN, Hunger SP, Steinherz PG, Gaynon PS, Seibel NL, Children's Oncology G. 2012. Effect of alternate-week versus continuous dexamethasone scheduling on the risk of osteonecrosis in paediatric patients with acute lymphoblastic leukaemia: results from the CCG-1961 randomised cohort trial. Lancet Oncol, 13 (9):906-915.

McAvoy S, Baker KS, Mulrooney D, Blaes A, Arora M, Burns LJ, Majhail NS. 2010. Corticosteroid dose as a risk factor for avascular necrosis of the bone after hematopoietic cell transplantation. Biol Blood Marrow Transplant, 16 (9):1231-1236.

Miettunen PM, Lafay-Cousin L, Guilcher GM, Nettel-Aguirre A, Moorjani V. 2012. Widespread osteonecrosis in children with leukemia revealed by whole-body MRI. Clin Orthop Relat Res, 470 (12):3587-3595.

Mirowitz SA, Apicella P, Reinus WR, Hammerman AM. 1994. MR imaging of bone marrow lesions: relative conspicuousness on T1-weighted, fat-suppressed T2-weighted, and STIR images. AJR Am J Roentgenol, 162 (1):215-221.

Mitchell DG, Rao VM, Dalinka MK, Spritzer CE, Alavi A, Steinberg ME, Fallon M, Kressel HY. 1987. Femoral head avascular necrosis: correlation of MR imaging, radiographic staging, radionuclide imaging, and clinical findings. Radiology, 162 (3):709-715.

Mohan S, Moineddin R, Chavhan GB. 2015. Pediatric whole-body magnetic resonance imaging: Intra-individual comparison of technical quality, artifacts, and fixed structure visibility at 1.5 and $3 \mathrm{~T}$. Indian J Radiol Imaging, 25 (4):353-358.

Möller HE. 2006. Grundlagen der MRT. In: Rummeny EJ, Reimer P, Heindel W, Hrsg. Ganzkörper-MR-Tomographie. Zweite Aufl. Stuttgart: Georg Thieme Verlag KG.

Nievelstein RA, Littooij AS. 2015. Whole-body MRI in paediatric oncology. Radiol Med.

Niinimaki RA, Harila-Saari AH, Jartti AE, Seuri RM, Riikonen PV, Paakko EL, Mottonen MI, Lanning M. 2007. High body mass index increases the risk for osteonecrosis in children with acute lymphoblastic leukemia. J Clin Oncol, 25 (12):1498-1504.

Niinimaki RA, Harila-Saari AH, Jartti AE, Seuri RM, Riikonen PV, Paakko EL, Mottonen MI, Lanning M. 2008. Osteonecrosis in children treated for lymphoma or solid tumors. J Pediatr Hematol Oncol, 30 (11):798-802.

Ozel BD, Ozel D, Ozkan F, Halefoglu AM. 2016. Diffusion-weighted magnetic resonance imaging of femoral head osteonecrosis in two groups of patients: Legg-Perthes-Calve and Avascular necrosis. Radiol Med, 121 (3):206-213.

Padhye B, Dalla-Pozza L, Little D, Munns C. 2016. Incidence and outcome of osteonecrosis in children and adolescents after intensive therapy for acute lymphoblastic leukemia (ALL). Cancer Med, 5 (5):960-967.

Papakostidis C, Tosounidis TH, Jones E, Giannoudis PV. 2016. The role of "cell therapy" in osteonecrosis of the femoral head. A systematic review of the literature and metaanalysis of 7 studies. Acta Orthop, 87 (1):72-78.

Reimer P, Parizel PM, Stichnoth F-A. 2003. Klinische MR-Bildgebung: Eine praktische Anleitung. Zweite Aufl. Berlin Heidelberg: Springer-Verlag.

Roth A, Beckmann J, Smolenski U, Fischer A, Jager M, Tingart M, Rader C, Peters KM, Reppenhagen S, Noth U, Heiss C, Maus U. 2015. [S3 guideline. Part 2: NonTraumatic Avascular Femoral Head Necrosis in Adults - Untreated Course and Conservative Treatment]. Z Orthop Unfall, 153 (5):488-497.

Saini A, Saifuddin A. 2004. MRI of osteonecrosis. Clin Radiol, 59 (12):1079-1093.

Schaefer JF, Kramer U. 2011. [Whole-body MRI in children and juveniles]. Rofo, 183 (1):2436.

Scherer A, Engelbrecht V, Krauth KA, Bernbeck B, Reinwand U, Willers R, Gobel U, Modder U. 2001. [MRI follow-up study of aseptic osteonecrosis (AON) in children treated with chemotherapy for malignant diseases]. Klin Padiatr, 213 (2):56-62.

Sharma S, Leung WH, Deqing P, Yang J, Rochester R, Britton L, Neel MD, Ness KK, Kaste SC. 2012. Osteonecrosis in children after allogeneic hematopoietic cell 
transplantation: study of prevalence, risk factors and longitudinal changes using MR imaging. Bone Marrow Transplant, 47 (8):1067-1074.

Socie G, Cahn JY, Carmelo J, Vernant JP, Jouet JP, Ifrah N, Milpied N, Michallet M, Lioure B, Pico JL, Witz F, Molina L, Fischer A, Bardou VJ, Gluckman E, Reiffers J. 1997. Avascular necrosis of bone after allogeneic bone marrow transplantation: analysis of risk factors for 4388 patients by the Societe Francaise de Greffe de Moelle (SFGM). Br J Haematol, 97 (4):865-870.

Te Winkel ML, Pieters R, Wind EJ, Bessems JH, van den Heuvel-Eibrink MM. 2014.

Management and treatment of osteonecrosis in children and adolescents with acute lymphoblastic leukemia. Haematologica, 99 (3):430-436.

te Winkel ML, Pieters R, Hop WC, de Groot-Kruseman HA, Lequin MH, van der Sluis IM, Bokkerink JP, Leeuw JA, Bruin MC, Egeler RM, Veerman AJ, van den Heuvel-Eibrink MM. 2011. Prospective study on incidence, risk factors, and long-term outcome of osteonecrosis in pediatric acute lymphoblastic leukemia. J Clin Oncol, 29 (31):41434150.

von Stillfried E, Weber MA. 2014. [Aseptic osteonecrosis in children and adolescents]. Orthopade, 43 (8):750-757.

Zurlo JV. 1999. The double-line sign. Radiology, 212 (2):541-542. 


\section{Anhang}

\subsection{Zeitlicher Verlauf der Osteonekrosen bei den einzelnen Patienten}

\subsubsection{Patienten ohne Behandlung der Osteonekrosen}

Von den betrachteten Patienten erhielten 7 Kinder und Jugendliche im Verlauf keine Therapie hinsichtlich ihrer ON. In den Diagrammen entspricht der Zeitpunkt „0 Monate“ dem Datum, an dem in einer GK-MRT erstmals ON nachgewiesen werden konnten.

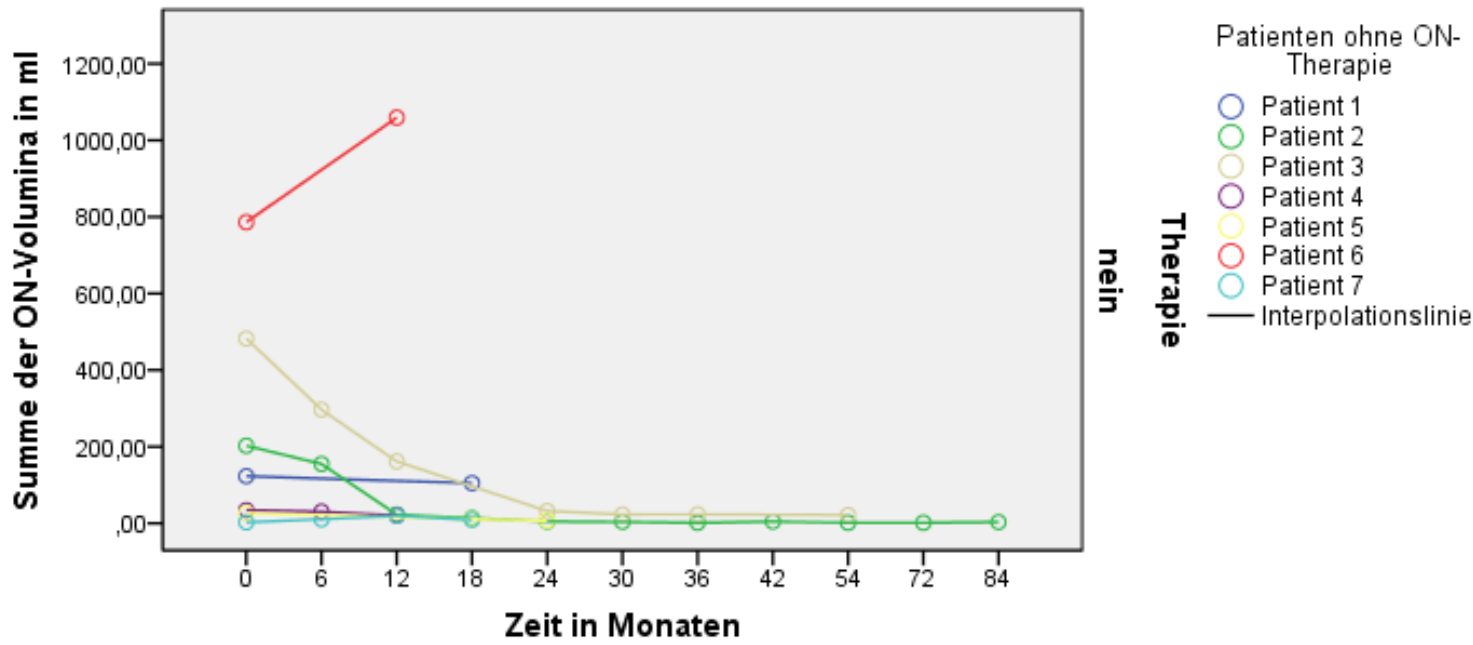

Abbildung 22: Zeitlicher Verlauf der Summe der ON-Volumina bei Patienten ohne ONTherapie

Patient 1 (weiblich, Alter bei Diagnose: 17,6 Jahre): 35,7 Monate nach der Diagnose (Alter: 20,6 Jahre) wurde erstmals eine GK-MRT zur Verlaufskontrolle durchgeführt, in der auch ON gesehen wurden. Im Verlauf wurde ein weiteres GK-MRT nach 18 Monaten durchgeführt. Die Anzahl der ON mit 19 Lokalisationen blieb stabil. Die Summe der Volumina aller ON nahm um $15 \%$ von $123 \mathrm{ml}$ auf $105 \mathrm{ml}$ ab. Die Patientin war über den ganzen Verlauf asymptomatisch.

Patient 2 (weiblich, Alter bei Diagnose: 16,1 Jahre): In der GK-MRT zur initialen Diagnosestellung waren keine Läsionen zu sehen. Nach nur 2,2 Monaten konnten in der zur Verlaufskontrolle durchgeführten GK-MRT erstmals ON nachgewiesen werden. Der Verlauf der ON erstreckte sich über 84 Monate. Von initial 3 ON mit einem Gesamtvolumen von $203 \mathrm{ml}$ sank dieses nach 11 Monaten auf $23 \mathrm{ml}$ bei immer noch 3 ON. Nach 84 Monaten war nur noch 1 ON mit einem Volumen von 3 $\mathrm{ml}$ erkennbar. Über den gesamten beobachteten Zeitraum konnte eine Abnahme der Summe der ON-Volumina von $99 \%$ verzeichnet werden. Die Patientin zeigte 5 
Monate nach dem initialen Nachweis von ON in der GK-MRT eine Schmerzsymptomatik in den Beinen, die einmalig dokumentiert wurde. Danach beklagte sie keine Beschwerden.

Patient 3 (weiblich, Alter bei Diagnose: 15,4 Jahre): 6,0 Monate nach Diagnosestellung (Alter: 15,9 Jahre) konnten zum ersten Mal ON in der GK-MRT gefunden werden. Diese Bildgebung wurde unter dem Verdacht auf ON durchgeführt. In einer externen Klinik konnten in einer MRT-Aufnahme bereits einen Monat nach Diagnosestellung der Grunderkrankung Nekrosen eruiert werden. Die Dauer der Verlaufsbetrachtung der ON bei dieser Patientin betrug 54 Monate. Die Anzahl der ON sank von initial 9 auf 6 Läsionen. Ebenfalls sank die Summe der ONVolumina um $96 \%$ von initial $483 \mathrm{ml}$ auf $22 \mathrm{ml}$ am Ende der Verlaufsbeurteilung. Schon 20 Monate nach dem ersten Nachweis von ON in der GK-MRT war das Gesamtvolumen von insgesamt 8 Nekrosen bei $32 \mathrm{ml}$. Ein halbes Jahr vor dem erstmaligen Nachweis der ON in einer GK-MRT beklagte die Patientin Schmerzen in beiden Kniegelenken. Später fand sich zusätzlich Schmerzsymptomatik im Becken und im linken Oberschenkel, die sich im Verlauf auf die gesamten Beine ausbreitete.

Patient 4 (weiblich, Alter bei Diagnose: 16,8 Jahre): 20,6 Monate nach Diagnosestellung (Alter: 18,5 Jahre) traten erstmals ON in der GK-MRT auf. Diese GK-MRT wurde unter dem Verdacht auf ON durchgeführt. Die beiden initialen GKMRT-Aufnahmen zur Diagnosestellung und zur Verlaufskontrolle 2 Monate danach waren frei von ON. Der Verlauf der ON erstreckte sich über 12 Monate. Die Anzahl der ON mit 4 Läsionen blieb stabil. Das Gesamtvolumen der ON sank über den gesamten Beobachtungszeitraum um 40 \% von $34 \mathrm{ml}$ auf $21 \mathrm{ml}$. Die Patientin wies im gesamten Verlauf keine Schmerzen hinsichtlich der Läsionen auf.

Patient 5 (weiblich, Alter bei Diagnose: 12,8 Jahre): Erstmals traten ON 1,9 Monate nach Diagnosestellung (Alter: 13,0 Jahre) in der zur Verlaufskontrolle durchgeführten GK-MRT auf. In der initialen GK-MRT zur Diagnosestellung der Grunderkrankung waren keine Läsionen sichtbar. Die Verlaufsbeurteilung der ON bei dieser Patientin erstreckte sich über 24 Monate. Im initialen GK-MRT konnten 2 Läsionen mit einem Gesamtvolumen von $27 \mathrm{ml}$ gefunden werden. Diese verschwanden wieder. In der ersten Verlaufsbildgebung waren 2 neue Nekrosen mit einem Gesamtvolumen von 
$11 \mathrm{ml}$ sichtbar. Das Volumen dieser beiden ON sank nach 7 Monaten auf $7 \mathrm{ml}$. Über den gesamten Beobachtungszeitraum konnte eine Abnahme der Summe der ONVolumina um $75 \%$ verzeichnet werden. Nach der letzten Verlaufs-GK-MRT traten bei der Patientin erstmals Knochenschmerzen im Kniegelenk auf.

Patient 6 (männlich, Alter bei Diagnose: 17,8 Jahre): 2,1 Monate nach Diagnosestellung (Alter: 18,0 Jahre) wurden erstmals ON in der zur Verlaufskontrolle durchgeführten GK-MRT nachgewiesen. In der initialen Diagnosestellung mittels GKMRT waren keine Läsionen sichtbar. Bei diesem Patienten wurde nur eine weitere GK-MRT nach 12 Monaten durchgeführt. Die Anzahl der Nekrosen stieg von 16 auf 18 Läsionen. Ebenfalls nahm die Summe der ON-Volumina um $35 \%$ von $786 \mathrm{ml}$ auf $1060 \mathrm{ml}$ zu. Der Patient litt im gesamten Verlauf nie unter Knochenschmerzen aufgrund der Läsionen.

Patient 7 (männlich, Alter bei Diagnose: 13,9 Jahre): 3,7 Monate nach Diagnosestellung (Alter: 14,2 Jahre) konnten erstmals in einer zur Verlaufskontrolle durchgeführten GK-MRT ON detektiert werden. Die beiden initialen GK-MRTBildgebungen zur Diagnosestellung sowie zur Verlaufskontrolle 2 Monate danach waren frei von Nekrosen. Der beurteilbare Verlauf der ON erstreckte sich über 18 Monate. Nach initial 2 gefundenen ON mit einem Gesamtvolumen von $3 \mathrm{ml}$ konnte nach einem halben Jahr nur noch eine Läsion nachgewiesen werden. Die 2 ON, die zu Beginn in der Bildgebung gefunden wurden, waren in der ersten Verlaufs-GKMRT nicht mehr zu sehen. Dafür wurde aber eine neue Läsion entdeckt, die bei ihrem ersten Nachweis ein Volumen von $11 \mathrm{ml}$ aufwies. Dieses nahm nach einem halben Jahr auf $20 \mathrm{ml} \mathrm{zu}$ und fiel dann wieder auf $9 \mathrm{ml}$ ab. Über den gesamten Beobachtungszeitraum erfolgte eine Zunahme der Summe der ON-Volumina um 242 $\%$, die im absoluten Wert nur rund $6 \mathrm{ml}$ entsprach. Der Patient blieb im gesamten Verlauf asymptomatisch. 


\subsubsection{Patienten mit Behandlung der Osteonekrosen}

Von den betrachteten 15 Patienten erhielten 8 Kinder und Jugendliche im Verlauf mindestens eine Behandlung hinsichtlich ihrer ON.

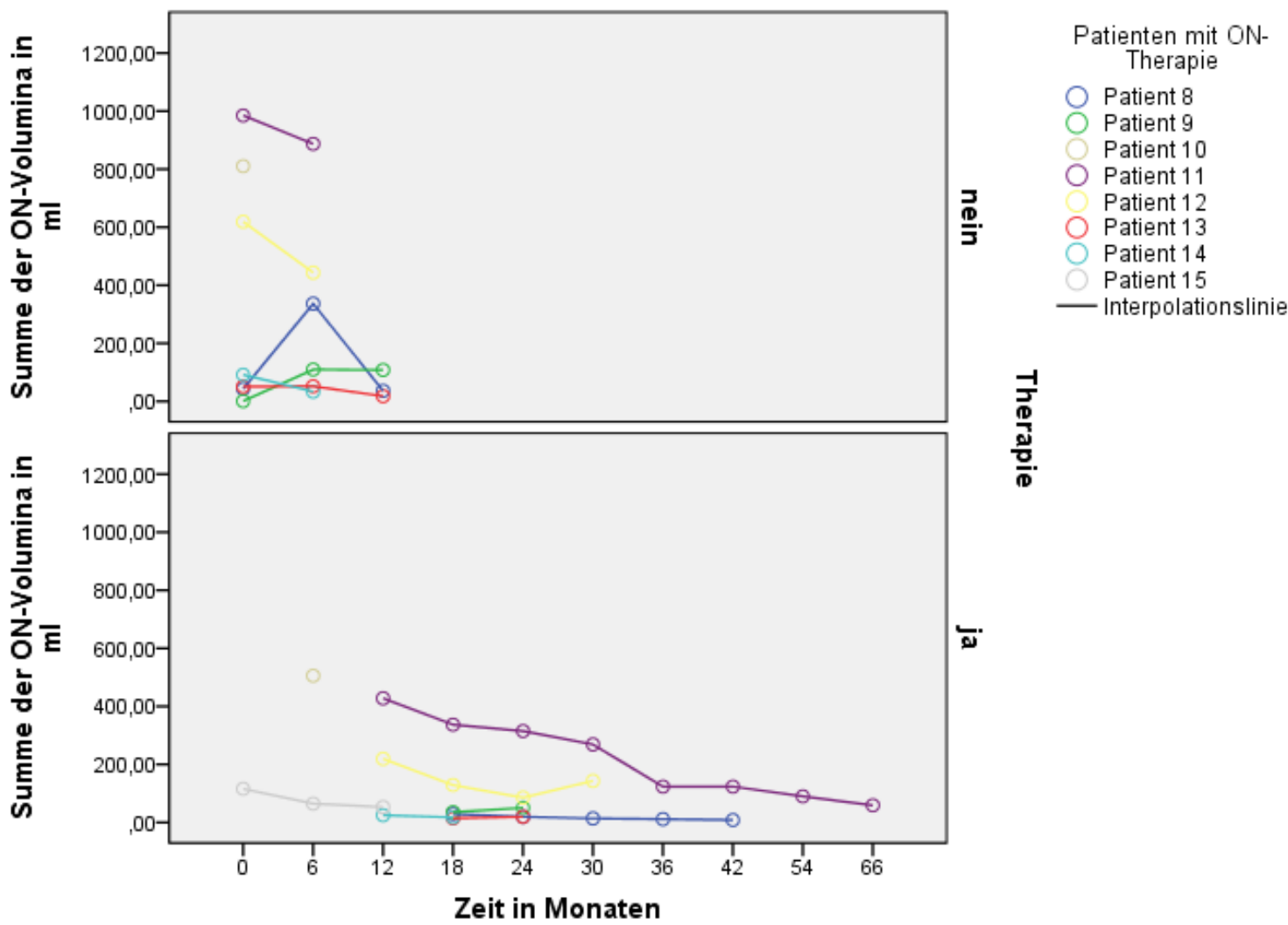

Abbildung 23: Zeitlicher Verlauf der Summe der ON-Volumina bei Patienten mit ONTherapie

Patient 8 (männlich, Alter bei Diagnose: 14,9 Jahre): 2,0 Monate nach Diagnosestellung (Alter: 15,1 Jahre) konnten in einer GK-MRT erstmals ON gesehen werden. Diese Bildgebung wurde unter dem Verdacht auf ON durchgeführt. Die initiale GK-MRT zur Diagnosestellung der Grunderkrankung war frei von Läsionen. Die Verlaufsbetrachtung der ON für diesen Patienten betrug 42 Monate. Initial konnten 3 Nekrosen mit einem Gesamtvolumen von $43 \mathrm{ml}$ gefunden werden. 3 Monate später stieg die Anzahl der ON auf 8 mit einer Summe der Volumina von 337 $\mathrm{ml}$. Noch vor der Therapie hinsichtlich der ON sank die Anzahl auf 5 ON mit einem Volumen von $37 \mathrm{ml}$. Ein Jahr nach dem erstmaligen Nachweis von ON in einer GKMRT beklagte der Patient Schmerzen im rechten Knie und es wurde eine Therapie mit 4 Zyklen des Prostacyclin-Analogons llopost im monatlichen Abstand durchgeführt. Vor dieser Therapie fanden sich in der Bildgebung $5 \mathrm{ON}$ mit einem Gesamtvolumen von $37 \mathrm{ml}$. Dies entsprach einem Abfall der Summe der ONVolumina vor der Therapie um 15 \%. Zum Ende der lloprostbehandlung summierten 
sich die Volumina der insgesamt nur noch $3 \mathrm{ON}$ auf $29 \mathrm{ml}$. 21 Monate nach dem initialen Nachweis von ON in der Bildgebung konnten in der GK-MRT nur noch 2 ON gesehen werden. Es begann eine Zoledronat-Therapie mit einer supportiven Gabe von Calcium und Vitamin D über 6 Monate. Die Medikamentengabe erfolgte alle 4 Wochen. In dieser Zeit sank das Volumen der 2 Nekrosen von 20 auf $14 \mathrm{ml}$. Im weiteren Verlauf war eine Läsion rückläufig. Die verbliebene Nekrose besaß am Ende der Verlaufsbetrachtung ein Volumen von $8 \mathrm{ml}$. Somit war eine Abnahme der Summe der ON-Volumina unter der Therapie im Vergleich zu vor Beginn des ersten Iloprostzyklus um 77 \% zu verzeichnen. Zum Zeitpunkt der letzten GK-MRT beklagte der Patient keine Schmerzen mehr.

Patient 9 (weiblich, Alter bei Diagnose: 17,6 Jahre): In der ersten GK-MRT zum Diagnostizieren der Grunderkrankung konnten auch ON gesehen werden. Die Verlaufsbeurteilung der ON konnte bei dieser Patientin über 24 Monate erfolgen. In der ersten GK-MRT mit ON-Nachweis fand sich nur eine ON mit einem Volumen von 0,9 ml. Nach einem halben Jahr konnten bereits 6 ON gesehen werden. Diese 6 ON wiesen nach 8 Monaten, vor Beginn der lloprosttherapie, ein Gesamtvolumen von $108 \mathrm{ml}$ auf. Über 11 Monate erhielt diese Patientin 8 Therapiezyklen mit dem Prostacyclin-Analogon. Schon 7 Monate nach Beginn der Therapie konnte ein Rückgang der ON auf 5 Läsionen mit einem Gesamtvolumen von $36 \mathrm{ml}$ verzeichnet werden. Nach Ende der Therapie wurde allerdings noch eine neue Nekrose gefunden und das Volumen stieg auf $51 \mathrm{ml}$. Im Vergleich zu vor Beginn der Therapie nahm das Gesamtvolumen aller ON um 53 \% ab. Die Patientin zeigte im gesamten Verlauf keine Schmerzsymptomatik.

Patient 10 (männlich, Alter bei Diagnose: 16,7 Jahre): 9,6 Monate nach Diagnosestellung (Alter: 17,5 Jahre) konnten zum ersten Mal ON in einer zur Verlaufskontrolle durchgeführten GK-MRT gesehen werden. In den initialen GKMRT-Aufnahmen zur Diagnosestellung der Grunderkrankung sowie 2 Monate vor dem Nachweis der ON waren keine Läsionen sichtbar. 8 Monate nach der Diagnosestellung trat bei dem Jungen zusätzlich ein Rezidiv auf. Die Verlaufsbetrachtung der ON für diesen Patienten erfolgte nur über 6 Monate. Zwischen den beiden GK-MRT-Aufnahmen erhielt der Patient neben einem Zyklus Iloprost auch Calcium und Vitamin D substituiert. Zu beiden Zeitpunkten wurden 10 
Läsionen gefunden. Das Gesamtvolumen dieser Nekrosen nahm unter der Therapie von $811 \mathrm{ml}$ um 38 \% auf $506 \mathrm{ml}$ ab. Der Patient war über den gesamten Verlauf asymptomatisch.

Patient 11 (männlich, Alter bei Diagnose: 13,7 Jahre): Zum ersten Mal traten ON in einer zur Verlaufskontrolle durchgeführten GK-MRT 1,9 Monate nach Diagnosestellung (Alter: 13,8 Jahre) auf. Die initiale GK-MRT zum Diagnostizieren der Grunderkrankung war frei von Läsionen. Die Dauer der Verlaufsbeurteilung für diesen Patienten betrug 66 Monate. Initial fanden sich 11 ON mit einem Gesamtvolumen von $985 \mathrm{ml}$. Im Verlauf ohne Therapie nahm die Anzahl der ON auf 10 Läsionen mit einem Abfall der Summe der Volumina aller ON um $10 \%$ auf $887 \mathrm{ml}$ ab. 4 Monate nach dem ersten Nachweis von ON in einer GK-MRT beklagte der Patient Schmerzen in beiden Kniegelenken und Füßen. Daraufhin begann eine Iloprosttherapie mit zunächst 2 Zyklen im monatlichen Abstand und einem Zyklus 3 Monate später. Ebenfalls erfolgte eine Anbohrung der Beine beidseits. Nach diesen Behandlungen, was im zeitlichen Verlauf 10 Monate nach dem initialen ON-Auftreten in der GK-MRT entsprach, konnten in einer Verlaufsbildgebung nur noch 5 ON mit einem Gesamtvolumen von $428 \mathrm{ml}$ gefunden werden. Im weiteren Verlauf traten wieder Schmerzen in den Fußgelenken als auch in beiden Oberschenkeln auf. 28 Monate nach dem ersten Zeitpunkt der Sichtbarkeit von ON in der GK-MRT wurde nochmals eine Anbohrung am linken Unterarm mit zusätzlicher einmaliger Gabe des Prostacyclin-Analogons Iloprost durchgeführt. Bis zum Ende der Verlaufsbetrachtung nahm die Anzahl der ON auf 3 Läsionen sowie deren Gesamtvolumen auf $59 \mathrm{ml}$ ab. Im Vergleich zu vor dem Beginn der ersten Zyklen der Iloprosttherapie sank die Summe der ON-Volumina um $93 \%$.

Patient 12 (männlich, Alter bei Diagnose: 17,7 Jahre): 1,9 Monate nach Diagnosestellung (Alter 17,9 Jahre) waren erstmals ON in einer zur Verlaufskontrolle durchgeführten GK-MRT nachzuweisen. Die beiden initialen GK-MRT-Aufnahmen zur Diagnosestellung der Grunderkrankung sowie 2 Monate vor dem Nachweis der ON waren frei von Läsionen. Die Dauer der Verlaufsbetrachtung betrug bei diesem Patienten 30 Monate. Beim ersten Nachweis von ON in der GK-MRT konnten 13 Läsionen mit einem Gesamtvolumen von $619 \mathrm{ml}$ gefunden werden. 2 Monate später beklagte der Patient erstmals Knochenschmerzen in beiden Beinen. Ein viertel Jahr 
nach der ersten GK-MRT mit Nekrosennachweis fand sich in der Bildgebung trotz einem Anstieg der Anzahl der ON auf 22 Läsionen eine Abnahme der Summe der Volumina um $28 \%$ auf $443 \mathrm{ml}$. 5 Monate nach dem initialen ON-Auftreten in der GKMRT erhielt der Patient 11 Zyklen Iloprosttherapie über 13 Monate. Nach dieser Behandlungsphase konnte eine Abnahme der Anzahl auf 16 ON mit einem Gesamtvolumen von $129 \mathrm{ml}$ verzeichnet werden. Im folgenden Verlauf blieb die Anzahl der ON stabil. Die Summe der ON-Volumina sank 22 Monate nach dem initialen ON-Nachweis in der Bildgebung zunächst auf $86 \mathrm{ml}$ und stieg dann wieder auf $143 \mathrm{ml}$ an. Im Vergleich zum Gesamtvolumen der ON vor Beginn der lloprosttherapie sank dieses unter der Behandlung um $68 \%$.

Patient 13 (männlich, Alter bei Diagnose: 12,4 Jahre): Zum ersten Mal traten ON in einer zur Verlaufskontrolle durchgeführten GK-MRT 2,1 Monate nach Diagnosestellung (Alter: 12,6 Jahre) auf. In der initialen GK-MRT zum Diagnostizieren der Grunderkrankung waren keine Nekrosen sichtbar. Die Verlaufsbetrachtung der $\mathrm{ON}$ für diesen Patienten erstreckte sich über 24 Monate. Beim ersten Nachweis von ON in der GK-MRT fanden sich 6 Läsionen mit einem Gesamtvolumen von $51 \mathrm{ml}$. Vor Beginn der Therapie nahm die Anzahl auf $3 \mathrm{ON}$ ab. Das Gesamtvolumen sank um $65 \%$ auf $18 \mathrm{ml}$. 15 Monate nach dem initialen Auftreten von $\mathrm{ON}$ in der Bildgebung erfolgte beim Patienten eine Anbohrung beider Füße mit zusätzlicher Gabe des Prostacyclin-Analogons Iloprost. Die Anzahl der ON blieb im folgenden Verlauf konstant. Die Summe der Volumina sank zunächst minimal auf $14 \mathrm{ml}$ und stieg dann auch wieder nur gering auf $20 \mathrm{ml}$ an. Insgesamt war eine Zunahme des Gesamtvolumens der ON um 10 \% während der Therapie zu verzeichnen. Der Patient war im gesamten Verlauf asymptomatisch.

Patient 14 (männlich, Alter bei Diagnose: 14,4 Jahre): 5,8 Monate nach Diagnosestellung (Alter: 14,9 Jahre) konnten erstmals ON in einer zur Verlaufskontrolle durchgeführten GK-MRT nachgewiesen werden. Die initiale GKMRT zur Diagnosestellung der Grunderkrankung war frei von Läsionen. Für diesen Patienten konnte der Verlauf der Summe der ON-Volumina über 18 Monate analysiert werden. In der ersten GK-MRT mit ON-Auftreten konnten 6 Nekrosen mit einem Gesamtvolumen von $92 \mathrm{ml}$ gefunden werden. 2 Monate später wurde der Patient mit Schmerzen in beiden Kniegelenken symptomatisch. 9 Monate nach dem 
initialen ON-Nachweis in der GK-MRT beklagte der Patient erneut Knochenschmerzen in beiden Kniegelenken, dabei mehr im linken als im rechten Knie. Daraufhin erfolgte supportiv die Substitution von Calcium und Vitamin D über 6 Monate. Vor der Therapie fanden sich in der GK-MRT 4 ON mit einem Gesamtvolumen von $33 \mathrm{ml}$ und somit eine Abnahme der Summe der ON-Volumina um $64 \%$ ohne Therapie. Nach der Substitution sank die Anzahl der ON auf 3 Läsionen und das Gesamtvolumen nahm unter der Behandlung im Vergleich zu vor der Therapie um $47 \%$ auf $18 \mathrm{ml}$ ab.

Patient 15 (weiblich, Alter bei Diagnose: 16,3 Jahre): 8,3 Monate nach Diagnosestellung (Alter: 17,0 Jahre) traten erstmals ON in einer zur Verlaufskontrolle durchgeführten GK-MRT auf. Die initiale GK-MRT zur Diagnosestellung der Grunderkrankung war frei von Läsionen. Der Verlauf der ON-Volumina wurde bei dieser Patientin über 12 Monate begutachtet. Schon vor dem ersten Nachweis von ON in der GK-MRT erhielt die Patientin supportiv täglich 1000 IE Vitamin D. 2 Monate nach Therapieende fanden sich zum ersten Mal 2 ON in der GK-MRT mit einem Gesamtvolumen von $116 \mathrm{ml}$. 2 Monate nach der ersten Bildgebung mit ONNachweis beklagte die Patientin Schmerzen in beiden Kniegelenken. Während der 9 Monate bis zur letzten vorhandenen GK-MRT, in der ebenfalls 2 ON mit einem verringerten Gesamtvolumen von $53 \mathrm{ml}$ zu sehen waren, wurde der Patientin nochmals über 3 Monate Calcium und Vitamin D substituiert. Die Abnahme der Summe der ON-Volumina über den gesamten Verlauf betrug $54 \%$. 


\subsection{Abbildungsverzeichnis}

Abb. 1: Geschlechterverteilung in den einzelnen Patientengruppen

Abb. 2: Osteonekrose in der rechten proximalen Tibiametaphyse bei einem 17-jährigen Jungen mit Morbus Hodgkin - Darstellung in der T1-SPACE- (a), T2-HASTE-IR-

(b) und T1-SPACE-SPAIR-Sequenz mit Kontrastmittel (c)

Abb. 3: Indikationen für die Durchführung der ersten GK-MRT .

Abb. 4: Lagerung des Patienten und Positionierung der Spulen für eine GK-MRT im MRTGerät Siemens Avanto (Universitätsklinikum Jena) .................................................. 31

Abb. 5: Definition der Lage der Osteonekrosen in den langen Röhrenknochen ...................... 33

Abb. 6: Aufteilung der Lokalisationen der Osteonekrosen nach Knochen (rechts) und gelenknahen Knochenregionen (links)

Abb. 7: Häufigkeit des Auftretens der Osteonekrosen in der GK-MRT je nach betrachteter Patientengruppe

Abb. 8: Aufteilung der Patienten mit Osteonekrosen in der GK-MRT bezüglich der

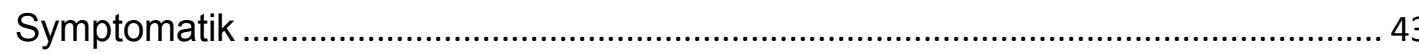

Abb. 9: Aufteilung der Lokalisationen der $\mathrm{ON}$ in Abhängigkeit von den Extremitäten........... 44

Abb. 10: Aufteilung der Lokalisationen der ON in Abhängigkeit von der Körperseite ............. 45

Abb. 11: Anzahl der betroffenen Knochen und gelenknahen Knochenregionen durch Osteonekrosen aufgeteilt in die jeweiligen Patientengruppen ................................... 45

Abb. 12: Verteilung der Osteonekrosen in den um das Kniegelenk liegenden Knochen........ 47

Abb. 13: Anzahl der Osteonekrosen in der jeweiligen Lage in den langen Röhrenknochen aufgeteilt in die jeweiligen Patientengruppen

Abb. 14: Anzahl der Osteonekrosen mit Anteil an der Epiphyse mit und ohne Gelenkflächenbeteiligung aufgeteilt in die jeweiligen Patientengruppen

Abb. 15: Kaplan-Meier-Kurven zur Darstellung des Einflussfaktors Alter bei der Diagnose der Grunderkrankung auf das Auftreten von Osteonekrosen in der GK-MRT bei Patienten ohne SZT

Abb. 16: Kaplan-Meier-Kurven zur Darstellung des Einflussfaktors Alter bei der ersten SZT auf das Auftreten von Osteonekrosen in der GK-MRT bei Patienten mit SZT ......... 52

Abb. 17: Kaplan-Meier-Kurven zur Darstellung des Einflussfaktors Geschlecht auf das Auftreten von Osteonekrosen in der GK-MRT bei Patienten ohne SZT ...

Abb. 18: Kaplan-Meier-Kurven zur Darstellung des Einflussfaktors Geschlecht auf das Auftreten von Osteonekrosen in der GK-MRT bei Patienten mit SZT

Abb. 19: Kaplan-Meier-Kurven zur Darstellung des Einflussfaktors Höhe der kumulativen Steroiddosis auf das Auftreten von Osteonekrosen in der GK-MRT bei Patienten ohne SZT...

Abb. 20: Zeitlicher Verlauf der Osteonekrosen $(\rightarrow)$ einer zur Diagnosestellung 15-jährigen Patientin ohne Therapie der Läsionen (Aufnahme a: Verdacht auf ON bei Schmerzen/erstes GK-MRT mit ON; b: 27 Monate danach; c: 52 Monate danach)

Abb. 21: Zeitlicher Verlauf der Osteonekrosen $(\rightarrow)$ bei einem zur Diagnosestellung 17jährigen Patienten mit Therapie der Läsionen (Aufnahme a: Diagnosestellung Morbus Hodgkin/erstes GK-MRT mit ON; b: 11 Monate danach unter Iloprosttherapie; c: 27 Monate danach (nach der Therapie)).

Abb. 22: Zeitlicher Verlauf der Summe der ON-Volumina bei Patienten ohne ON-Therapie. 95 Abb. 23: Zeitlicher Verlauf der Summe der ON-Volumina bei Patienten mit ON-Therapie .... 98 


\subsection{Tabellenverzeichnis}

Tabelle 1: ARCO-Klassifikation der atraumatischen Femurkopfnekrose (von Stillfried und Weber 2014)................................................................................................... 18

Tabelle 2: Aufteilung der Patienten in Abhängigkeit von der Höhe der verabreichten

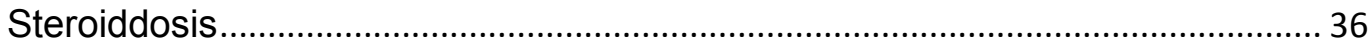

Tabelle 3: Daten zum Qualitätsscore (QS) in den einzelnen Sequenzen (SD = Standardabweichung; KM = Kontrastmittel) …................................................. 39

Tabelle 4: Vergleich der Qualitätsscore (QS) zwischen den einzelnen Sequenzen (KM =

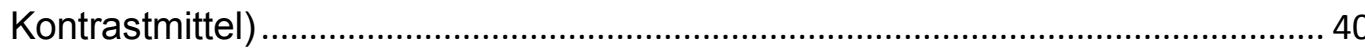

Tabelle 5: Daten zur diagnostischen Sicherheit (DS) in den einzelnen Sequenzen (SD = Standardabweichung; KM = Kontrastmittel) ...................................................... 40

Tabelle 6: Vergleich der Werte zur diagnostischen Sicherheit (DS) zwischen den Sequenzen (KM = Kontrastmittel)

Tabelle 7: Häufigkeiten des Auftretens der Osteonekrosen in der GK-MRT bei Patienten ohne SZT je nach Grunderkrankung

Tabelle 8: Anzahl der Osteonekrosen pro Patient (SD = Standardabweichung) 44

Tabelle 9: Häufigste Lokalisationen der Osteonekrosen aufgeteilt in die jeweiligen Patientengruppen

Tabelle 10: Anzahl der von Osteonekrosen betroffenen Knochenbereiche um ein Gelenk pro Patient (SD = Standardabweichung)

Tabelle 11: Anzahl der Patienten in den jeweiligen Patientengruppen nach Höhe der verabreichten kumulativen Steroiddosis

Tabelle 12: Anzahl der Patienten in den jeweiligen Patientengruppen in Abhängigkeit von der Art des verabreichten Steroids

Tabelle 13: Vergleich des Auftretens von ON in der GK-MRT in Abhängigkeit von der Art des verabreichten Steroids

Tabelle 14: Anzahl der Patienten in den jeweiligen Patientengruppen nach Höhe der verabreichten Prednisolondosis

Tabelle 15: Vergleich des Auftretens von ON in der GK-MRT in Abhängigkeit von der Höhe der verabreichten Prednisolondosis

Tabelle 16: Anzahl der Patienten in den jeweiligen Patientengruppen nach Höhe der verabreichten Dexamethasondosis

Tabelle 17: Vergleich des Auftretens von ON in der GK-MRT in Abhängigkeit von der Höhe der verabreichten Dexamethasondosis 


\subsection{Ehrenwörtliche Erklärung}

Hiermit erkläre ich,

dass mir die Promotionsordnung der Medizinischen Fakultät der Friedrich-SchillerUniversität bekannt ist,

ich die Dissertation selbst angefertigt habe und alle von mir benutzten Hilfsmittel, persönlichen Mitteilungen und Quellen in meiner Arbeit angegeben sind,

mich folgende Personen bei der Auswahl und Auswertung des Materials sowie bei der Herstellung des Manuskripts unterstützt haben: Univ. - Prof. Dr. med. HansJoachim Mentzel (Sektion Pädiatrische Radiologie, IDIR; Universitätsklinikum Jena), Dr. med. Karim Kentouche (Sektion Hämatologie und Onkologie, Klinik für Kinderund Jugendmedizin; Universitätsklinikum Jena), Dr.-Ing., M.Sc. Epidemiology Heike Hoyer (Institut für Medizinische Statistik, Informatik und Dokumentation; Universitätsklinikum Jena),

die Hilfe eines Promotionsberaters nicht in Anspruch genommen wurde und dass Dritte weder unmittelbar noch mittelbar geldwerte Leistungen von mir für Arbeiten erhalten haben, die im Zusammenhang mit dem Inhalt der vorgelegten Dissertation stehen,

dass ich die Dissertation noch nicht als Prüfungsarbeit für eine staatliche oder andere wissenschaftliche Prüfung eingereicht habe

und dass ich die gleiche, eine in wesentlichen Teilen ähnliche oder eine andere Abhandlung nicht bei einer anderen Hochschule als Dissertation eingereicht habe.

Ort, Datum

Unterschrift 